This item was submitted to Loughborough's Research Repository by the author.

Items in Figshare are protected by copyright, with all rights reserved, unless otherwise indicated.

Social skills training with young adolescents: group and individual approaches in a school setting

PLEASE CITE THE PUBLISHED VERSION

PUBLISHER

(C) Richard Bulkeley

LICENCE

CC BY-NC-ND 4.0

REPOSITORY RECORD

Bulkeley, Richard. 2019. "Social Skills Training with Young Adolescents: Group and Individual Approaches in a School Setting”. figshare. https://hdl.handle.net/2134/7121. 
This item is held in Loughborough University's Institutional Repository (https://dspace.lboro.ac.uk/) and was harvested from the British Library's EThOS service (http://www.ethos.bl.uk/). It is made available under the following Creative Commons Licence conditions.

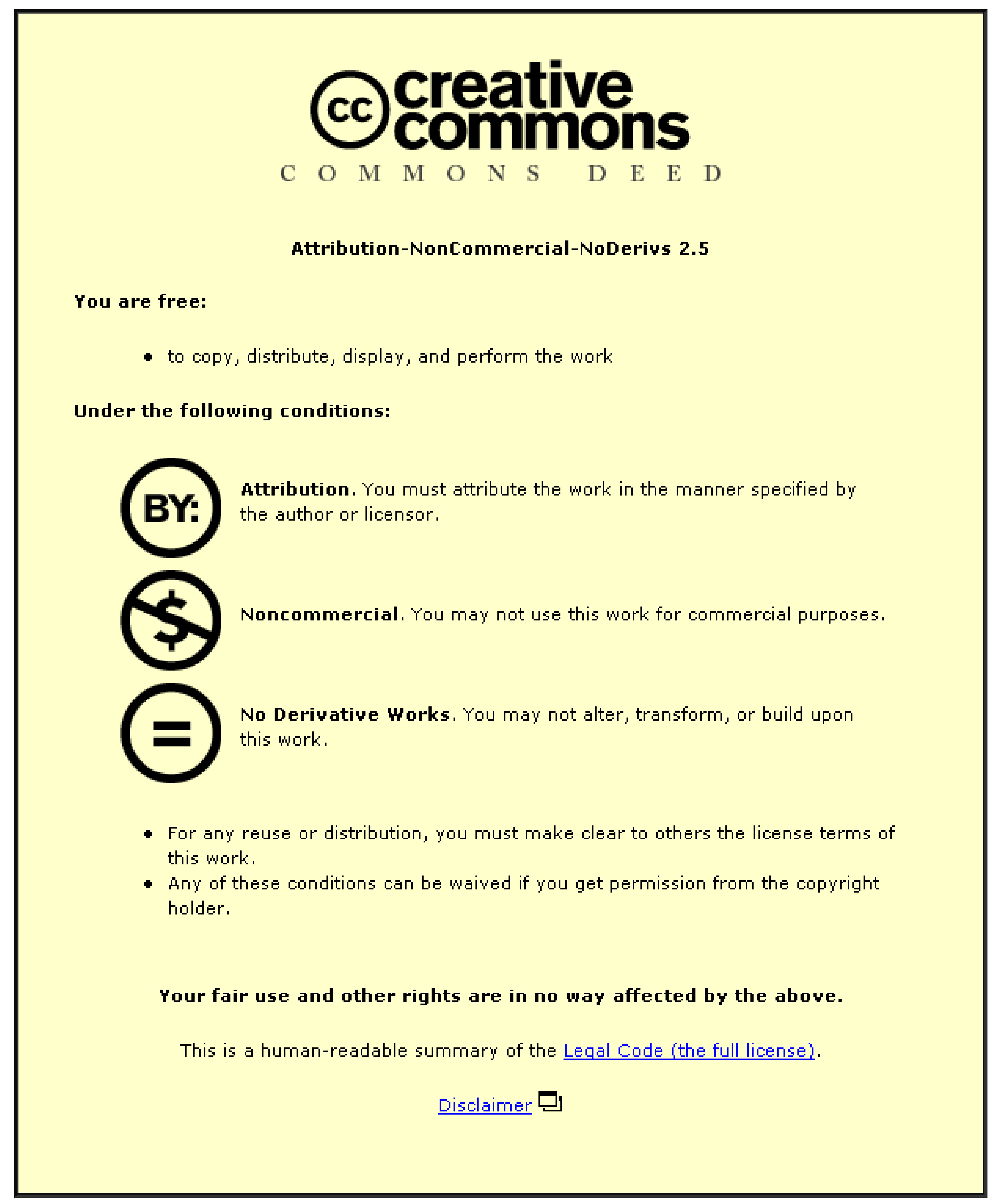

For the full text of this licence, please go to: http://creativecommons.org/licenses/by-nc-nd/2.5/ 
Social Skills Training with Young Adolescents: Group and

Individual Approaches in a School Setting

by

Richard Bulkeley

A Doctoral Thesis

Submitted in partial fulfilment of the requirements

for the award of

the Degree of Doctor of Philosophy of the Loughborough University of Technology

(C) copyright by Richard Bulkeley, 1991 


\section{Table of Contents}

\section{Synopsis}

\section{Chapter}

1.

Background to the Studies: A Review of the Literature

2.

The First Study: Small Group Training in School

3.

The Second Study: Individualised Group

Training

4.

Discussion of the studies

5.

Appendices

6.

References 
Synopsis

There has been relatively little research on social skills training with young adolescents. Yet adolescence is a key stage in the life cycle, and may well be important for the development of social skills. There is evidence that difficulty with the peer group early in life may be a risk factor for a range of personal problems at a later stage in the life cycle. The research programme sought to evaluate the use of group social skills training with young adolescents in a school setting, and to identify the most useful component.

The research consisted of two experiments, each of which involved the provision of a social skills training programme for young adolescents of mixed gender within the school timetable.

In the first experiment nine young adolescents aged 12-13 were identified as having social skills difficulty. The intervention took the form of a group programme of ten social skills training sessions with a strong individualised component. On measures of social competence and self-report significant gains were made which were maintained at six month follow-up. On a measure of sociometric status there was significant gain by the time of follow-up. A non-equivalent problem-free no-treatment control group (n = 10) of like age made no significant gains on any of the variables measured. There were significant differences between the control group and the treatment group in social competence and sociometric status prior to treatment, but none post treatment nor at follow-up. Using MANOVA a main 
effect for time was found for the combined measures of social competence and self-report.

In the second experiment 41 young adolescents aged 12-13 were allocated to an individualised training (IT) Group ( $\mathrm{n}=14)$, a standardised training (ST) Group ( $\mathrm{n}=13$ ) and a no treatment control group ( $\mathrm{n}=14$ ). Therapist effects were controlled by crossing over therapists, so that equal numbers of each group were trained by each pair of therapists. The programme ran over 2 years. A group comparison design taken together with single case designs provided evidence for the effectiveness of the interventions and for the relative superiority of individualised training over standardised training. Dependent measures included a self-report questionnaire, a sociometric questionnaire, a behavioural interview schedule, a role-taking test, a social assertiveness test, and a clinical change rating. There was no main effect for type of problem (rejected vs neglected) nor for subject gender. Data relating to therapist performance suggested that therapist effects might make a substantial difference to outcome.

It was concluded from the two experiments that the treatments were generally effective and able to produce generalisation, that the individualised approach worked rather better than the standardised approach, and that it was important to control for therapist effects. 
SOCIAL SKILLS TRAINING WITH YOUNG ADOLESCENTS

Chapter One

\section{Background to the studies}

\section{Table of Contents}

\section{Section.}

Title.

1.

The concept of social skills training.

2 . The social skills model.

3. Areas of application.

4. A developmental approach to social skills training.

5. Assessment for social skills training.

6. Intervention.

7. The assessment of outcome.

8. Planning for generalisation.

9. Assessment of generalisation.

10. Therapist effects.

11. The individualised approach.

12. Conclusions and implications.

13. Towards implementation. 
SOCIAL SKILLS TRAINING WITH YOUNG ADOLESCENTS

\section{Chapter One}

\section{Overview of Thesis}

In the first chapter, information relevant to the provision of social skills training to young adolescents is collated and reviewed. Issues such as assessment, the various approaches to intervention, and the question of how to intervene are discussed with reference to the literature. Two arguments are then developed; firstly that it may be useful to provide such input within a school-based setting through the medium of small groups; and secondly that within that context, the approach will work best if the social skills training is tailored, as far as possible, to the needs of individuals. Two studies are then reported which explore these propositions in some detail.

\section{The Concept of Social Skills Training}

Introduction

Three areas of research underpinned the "take-off" of social skills training in the late 1970s.

The first area was research into mental illness, emphasising the role of social functioning in recovery (eg Zigler and Philips, 1961). These authors found that the level of social competence in clients with psychological disorders had a considerable significance for outcome in the treatment of those disorders. 
The second main area was social learning theory, involving new applications of the learning model to human processes such as modelling and selfreinforcement (Bandura, 1977a). In the 1960s, clinical psychologists had already been experimenting with "behavioural" approaches, based on Skinnerian learning theory. This process was provided with further theoretical under-pinning by Bandura's development of social learning theory. Having previously taken an interest in detailed strategies such as "shaping" and "chaining" (Bandura, 1969), Bandura now, through social learning theory, applied the model more specifically to human cognitive processes; these included modelling, imitation, and self-reinforcement.

Thirdly, social psychology contributed the "social skills model" (Argyle, 1967). Argyle drew an analogy between social and motor skills. His model incorporated both motor and learning processes in a type of systemic analysis previously applied to ergonomics. A unit of social behaviour/learning is defined as a systemic process occurring in three phases. Firstly, an individual has or identifies a social goal, or subgoal. For example, he may identify a person with whom he wishes to make friends. Secondly, environmental cues relevant to the attainment of that goal are perceived; and in response to that perception, a "goal-directed" response occurs. For example, the "targeted" friend may smile in an encouraging manner, to which the individual may respond by approaching and initiating conversation. Finally, the individual's motor response may produce some change in the targeted friend. In the language of the model, this is described as "change in the environment" which is "fed back" to the individual so long as he correctly perceives and interprets it. In social learning theory terms, the response of the individual is "reinforced" if the targeted friend responds positively, and that positive response is correctly observed by the individual who duly makes appropriate plans for 
future interactions. These then are the three stages of a cycle of interaction which involves social learning, social cognition and social behaviour.

Other relevant developments were assertion training, (Wolpe, 1969) and research into "micro-skills", (Ekman and Friesen, 1969; Trower, 1967). In assertion training, behavioural techniques are used to "shape" assertive social responses; this was found helpful with neurotic individuals who lacked confidence and were not able to take the initiative in social situations. "Micro-skills" may be described as the behavioural "small change" of social interaction - eye contact, facial expression, gesture and the like. Social skills training programmes in the 1970s (Liberman et al., 1975: Trower, Bryant and Argy11, 1978) provided for instructions in microskills such as the following: facial expression, gesture, posture, spatial behaviour, bodily contact, physical appearance, non-verbal aspects of speech, and use of language or "speech acts" (Searle, 1975).

A number of types of people have been seen as particularly suitable for social skills training. Winter and Marzillier (1983) note the following commonly occurring social skill difficulties in their clients: poor conversation skills, lack of appropriate assertiveness, poor appearance, inability to form friendships, limited range of social activities, poor social sensitivity and low social self-esteem.

It is easiest to define social skills training in a behavioural (pragmatic) manner. "SST is an active, performance-based treatment. The core methods are modelling, verbal instructions, practice (role-play) and homework assignments. It can be done individually, or in groups. The number and spacing of treatment sessions depends on the nature of the clients 
involved, and the aims." (Shepherd, 1983, pp 11-12).

Winter and Marzillier (1983) present a case example of a young man of 23 who was initially diagnosed four years previously as suffering from a "phobic anxiety state with associated depression". Difficulty in interaction with others and concern about this seemed to be contributing to his anxiety and depression. The eldest of 6 children, he spent a good deal of time in bed, was socially isolated and anxious about making any social contact. Conventional psychiatric treatment produced little change and he was re-referred at the age of 23 with essentially the same problem. His mental state had in fact deteriorated in that he was now beginning to talk of suicide. Assessment indicated that he had difficulty in thinking of topics for conversation, and it was observed that his actual utterance in response to questions were indeed very limited. As a result he had become isolated from social groups, and now avoided interaction.

From an analysis of his limited conversational output, it was possible to construct behavioural "goals" for future work, namely to increase the frequency and duration of his utterances, whilst of course ensuring that these were socially acceptable and appropriate! To achieve this, role-play practice ("behavioural rehearsal") was required. In addition, some medium-term personal goals were agreed - the experience of having a girlfriend, socialising with workmates, and arranging to have riding lessons (following up this interest seemed likely to extend his range of social contact). These medium term goals were to be followed up and reviewed in individual therapy. At this stage, an assessment of his motivation was made and it was felt that he might have quite a strong commitment to cooperating with a treatment programme. 
After assessment, targets for treatment were agreed and two of these were tackled in a social skills training group while two were tackled individually.

This client was successfully treated through a programme which included a range of components. He received videotaped feedback on his conversational skills, joined an active social club, and attended 10 sessions of a mixed small group in which, together with other group members, he was given structured guidance or "shaping" in conversational skills such as questionasking. At an individual level, he was given guidance in modifying his appearance, since he ws of small stature and appeared "boyish". To deal with the girl-friend issue, he himself had the idea of joining a computer dating organisation. In individual sessions he was helped to prepare for the "dates", and again this took the form of rehearsal of possible conversational openings, questions, and "ending remarks".

In reference to social skills training in groups Winter and Marzillier state "We would only advocate a form of group training which is oriented towards individuals. In other words, individual training is provided within a group framework, allowing patients to learn from one another, to practise conversation together, and even to tackle agreed social activities together outside the clinical setting." (p 49.) 
From the above description, it is possible to derive a formulation of what actually happens in at least one form of social skills training. First, an individual is referred and assessed. If treatment is decided on, certain real life tasks or "targets" are agreed between trainer/therapist and client. The task might be to increase social interaction with workmates or to get a girlfriend. The trainer/therapist then acts as a consultant in advising on the behaviours which, if successfully carried out, will lead to accomplishment of these tasks or "goals", and proceeds to provide encouragement or "reinforcement" to the client for persisting in these behaviours. The therapist then is a kind of behavioural consultant, and success in the treatment presumably consists in attainment of the agreed goals and maintenance of the newly acquired behaviours after treatment ends. The model here outlined is considered a useful one by the present author, but clearly there could be other models for describing a social skills intervention. What is important is that there should be a clearly articulated model which has at least face validity.

The above formulation is consistent with the definition of skill given by Herbert (1986): "The utilisation of a complex set of behaviours in order to accomplish a task".

The model of social skills training so far elaborated may be extended to include work with children. Rinn and Markle (1979) give the following definition of social skills "A repertoire of verbal and non-verbal behaviours which enable children to affect the responses of others in such a way that desirable outcomes are attained and undesirable outcomes avoided without inflicting pain on others." In undertaking social skills training therefore the authors take as their aim the task of enhancing a child's repertoire by adding skills that the child appears to lack. In their 
analysis social skills are broken down into the following four areas: selfexpressive skills, other-enhancing skills, assertive skills, and communication skills. Self-expressive skills include the expression of feeling, accepting compliments, and making positive statements about oneself. Other-enhancing skills include stating positives about a best friend, stating genuine agreement with another's opinion, and praising others. Assertive skills include making simple requests, disagreeing with another's opinion, and denying unreasonable requests. Communication skills include conversing and interpersonal problem-solving.

Rinn and Markle have some reservations about the value of a clinical interview for assessing social skills. They do not find parents and teachers to be very good judges of a child's social skills. They do not consider that a child's responses in a clinical interview give a good indication of a child's range of behaviour in the natural environment, as the clinical interview is an artificial situation and the child's response to it may therefore be "situation specific".

From the above it would appear that broadly, the approach to social skills training is the same with children as it is with adults. However, both the content and the approach must be geared to the child's developmental level. There may be methodological differences, for example in the type of assesment which needs to be carried out. 


\section{The social skills model}

In this section the "Argyle model" is presented and a number of contraversial aspects of the social skills model are discussed.

\section{i) The Argyle model}

Argyle and Kendon (1967) set out a formulation whereby the sensorimotor skill training model might be applied to social interaction.

The individual is seen as an operator working towards a goal. Observing the effect of successive actions on the environment, the operator modifies performance in order to attain the goal.

Each cycle of the operation is performed in three phases - Perception, Translation, and Effector Processes.

In the Perception phase, the individual observes relevant aspects of the social environment and also receives feedback on his own previous actions. Jones and Thibaut (1950) suggest that an interactor's main requirement is for information relative to the adequate maintenance of a particular performance. Steiner (1955) reviews a number of studies which indicate that effectiveness in social performance is related to the accurate perception of relevant features of the situation.

In the Translation phase, the individual identifies the signal from the environment as requiring a particular action. In the acquisition of skill, it is important to acquire the facility to respond rapidly to signals with appropriate action. Here again the social skills model closely follows the 
sensorimotor skills acquisition model.

In the stage of effector processes, the individual responds at a number of levels. Firstly there is general orientation to the task; secondly, detailed plan of method, for example breaking up a business contact with a colleague into the separate phases of greeting, establishing rapport, dealing with item of business, and termination; thirdly "knacks and dexterities" which might include the sequence of acts of communication.

It is recognised that the interaction will involve at least two individuals and that the interactants will need to maintain a working consensus.

The performance of the effector processes may be analysed in terms of structural and dynamic features. Hall (1963) identifies the following stuctural components: posture, spatial orientation, distance, physical contact (whether present, and if so of what nature), eye contact, and communication by other sensory channels (thermal, olfactory). Distance sets may be divided into four types: intimate, casual/personal, social/consultative, and public (Hall, 1964).

Dynamic components include patterning of action in time (where action may include verbal participation); language and speech (Joos, 1962, identifies a range of linguistic styles from Frozen to Intimate); visual orientation, a number of expressive functions of eye contact being well established (power, affiliation, sexuality, fright/flight); bodily movement, and facial expression. Ekman and Friesen (1971) introduced the neuro-cultural theory of facial expression, whereby the pancultural or "natural" expressions of emotion by the facial musculature are held to be overlaid by cultural "display" patterns, so that they may be intensified, de-intensified, 
neutralized, or masked. Capacity to employ facial expression appropriately in social interaction is no doubt an important element of social competence, though research on this area is sparse.

Argyle and Kendon (1967) give some indication of how social competence may be measured. In the case of professional social skills, competence is related to the attainment of some combination of goals. In non-professional contexts, individuals may increase their capacity to act cooperatively and maintain frindly relationships. These characteristics are noticeably lacking in psychiatric patients. Argyle and Kendon (1967) suggest that social skills may be enhanced by roleplaying with video feedback and by other structred methods aimed at enhancing communication and social problem-solving.

\section{ii) Empirical definition.}

"Everyone seems to know what good and poor social skills are, but no one can define them adequately." (Curran, 1979, p 321.) It is possible to make a global assessment of someone's social skills, but difficult to identify the contribution made by individual elements (eg the use of eye contact). The variable which actually makes a difference may be quite subtle and difficult to detect by direct observation, eg sensitivity to changes in the behaviour of an interactant (Trower, 1979). Curran's criticism has not been adequately answered and we must not therefore suppose that social 
skills may be easily quantified and described; sometimes they may be, but often they can be expected to be quite elusive.

\section{iii) Situation specificity}

Trower (1979) makes the point that behaviour is determined by interaction of person and situation, rather than by characteristics of the person alone. Mischel (1968) reviewed personality theory and pointed out it's empirical weakness, emphasising that people behave variably according to situation. Jones and Nisbett (1971) came to similar conclusions in examining processes of attribution. They found that people are inclined to attribute too much behaviour to internal causes (the person) and too little to external causes (the situation). We must therefore always look at the interaction of personality with situation. Endler and Magnusson (1976) sum up the matter as follows: "Empirical results support an interactional view of behaviour, in which actual behaviour is determined by a continuous and multidirectional interaction between person and situation variables" ( $p$. 968).

There are a number of implications for the practicalities of social skills assessment and training. Clearly one error to avoid is that of making false predictions from the person-situation interaction observed in clinical assesment to the behaviour to be expected across a range of situations in "real life". Similarly, it must not be assumed that an improvement in the person-situation interactions observed in clinical treatment will necessarily guarantee a similar improvement in a different range of situations in the natural environment. Empirical evidence must be sought.

It is important to be clear as to which behaviours are more likely to be 
determined by personality, and therefore likely to be consistent across situations, and which are more likely ot be situation-dependent. We know that extraverts speak more rapidly, more loudly, and at greater length than introverts (Trower,1979) . Further, Trower, (1979) asserts that "Numerous studies highlight gender differences in behaviour" (p.25). We know that (in our culture, on average) women are more affiliative than men; they engage in more eye contact, adopt more proximal positions, smile more, selfdisclose more, and empathise more. With the same qualifications, men are more assertive, and take the initiative in conversations. Men and women appear to have different patterns of non-verbal communication in situations requiring assertion. Men adopt a closer position, assume a more relaxed posture, and use more touch; women are more tense in their posture, adopt a more distant position, and avoid touch.

Situation-dependent behaviour is considered to be most common in highly structured situations such as ceremonies (Trower, 1979). In casual encounters, the effect of personality is likely to be greater.

Behaviour is affected by social norms. In conversation, people repress behaviour that is not believed to conform to social rules, e.g the expression of anger and fear (Ekman, 1971) . Repression of emotion expressed via the face is most pervasive; most is "given away" via the lower parts of the body - the legs and feet. Here "censorship" is least effective (Ekman and Friesen, 1969).

There appear to be norms which apply to the stages of friendship making (Trower, 1979). Firstly there is the stage of visual perception where external cues are important. Secondly there is the stage of initial contact where a "catalogue" of information is exchanged; this enables individuals 
to place each other on the "social map", and determine what type of relationship might be possible. Thirdly there is the stage of affiliation which typically involves self-disclosure and accommodation.

Other norms have been identified in relation to "social routines" (Goffman, 1972). Goffman calls greetings and partings "supportive interchanges" and believes that they have a special importance. The basic strucure appears to be universal across cultures.

Argyle (1977) analyses situations into their essential components. These include "special moves" (passing food at a dinner party), goals, rules, roles (host and guest), "pieces" ("props", tools, or materials) and concepts.

It follows from the above brief review (the reader is referred to Trower, 1979, for a more detailed analysis) that a social skills trainer must make a thorough assessment of (i) relevant personality variables (ii) the clients ability to cope with the "universals" of social intercourse, such as greetings, partings, and making friends (iii) ability to cope with certain specific social situations which may be of importance at the current stage in the client's development le.g. "dating" in the case example given above.) In terms of theory, (ii) and (iii) are subsumed under the label "person-situation interaction". It is in these areas that the client will need to modify his behaviour patterns and achieve more social success. 
iv) "Behavioural" vs internal elements of social skill

Internal elements include the perceptual and translation processes suggested by Argyle (Argyle and Kendon, 1967) and also the physiological management of emotion, mentioned by McFall (1982). Ladd and Asher (1985), reviewing the child literature, employ the term "process variable", introducing the issue as follows: "Historically, research on the antecedents of status has focused on the overt behavioural style of children in their interaction with peers. Recent efforts, however, have been aimed at several process variables that might underlie children's ability to behave competently in social groups. These include children's knowledge of interaction strategies, children's goals in social situations, and chuildren's confidence in their ability to achieve interpersonal goals" (p.228).

The first process variable cited by Ladd and Asher - knowledge of interaction strategies - may be illustrated by the research of Asher and Renshaw (1981). These authors provide children wirth nine situations involving initiation or entry, maintenance of ongoing interaction, or resolution of a conflict. Unpopular children's ideas were found to be less friendly and effective. The finding suggests that unpopular children actually lack knowledge about how to behave in various social situations. 
This area of interest within the child field parallels the concerns of Trower (1984) in the adult field of social skills training. Basically Trower is concerned that as social skills trainers we should not act like dog-trainers (or indeed like Professor Higgins) in training our clients to leap successfully through various social hoops. He wishes to take issue with the more mechanistic, reductionist aspects of behaviourism. Trower addresses the matter on a philosophical plane. Trower sees behaviourism as based on the premise of logical positivism which affirms that only that which can be verified by observation is real. Trower challenges this premise which, in his view, undermines the true value of mental events in relation to physical events. Trower affirms that mental events are as significant as physical events, but of a different order.

This development is echoed by other incursions from the behavioural to the cognitive field. In a very influential paper, D'zurilla and Goldfried (1971) delineate a procedure whereby behaviour modification techniques may be applied to problem-solving. They suggest that there are five main stages to problem-solving: general orientation, problem formulation, generation of alternative solutions, choice of a suitable solution, and finally verification.

"Process variables" include emotional as well as cognitive entities. A key emotional factor for clients requiring social skills training is likely to be social anxiety. This is described by Winter and Marzillier (1983) in the adult literature as follows: "A degree of social anxiety is prevalent in society as a whole and a regular experience for most people. When this anxiety becomes extreme, it incapacitates the sufferer and renders social interaction aversive." Newton et a1.' (1983) compared 28 clients referred with social interaction difficulties with a non-clinical population. The 
most salient characteristic of the clinical group was found to be a very high level of social anxiety; this was more often related to low selfesteem then to inadequate social performance.

Clearly, then, the assessment of social anxiety is of extreme significance for social skills trainers. Santer (1983) reports on an interesting programme in which stress management and rational-emotive therapy are integrated with behavioural social skills training routines. This appeared to be extremely suitable in clinical work with psychiatric Day Patients.

In the literature, views diverge as to whether it is proper to pay attention to process variables in the normal course of social skills training. Argyle (1967) included the concepts of perception and cognition in the "social skills model". One might suppose that a social skill knowledge base (or repertoire of knowledge of interaction strategies) would be assumed by such a model, but it is not clear whether and how deficiencies in self esteem might be fitted into the model, which is very much of the "human operator" variety. Trower (1984) proposes a model of "generative social skill" in which the individual is assumed to become more competent in generating appropriate responses to the demands made by the social environment. - Yardley (1979) argues that the didactic component of social skills training is "absurd" and that the main effectiveness of SST is obtained through improving clients' self-esteem and their ability to focus on the world of others. A corollary of this view would be the proposition that process variables are a relevant area for research in social skills training. Curran (1979), on the other hand, confesses himself "ambivalent about the expansion of the construct of social skill to include cognitive processes." He later acknowledges his bias: "If we do not restrain ourselves, and put some limit on the construct of social skill, it 
will expand to include all human behaviour, and social skills training will soon come to mean any process which is capable of producing changes in human behaviour." (p 323). Some years later Curran et al. (1984) have reservations about the value of including cognitive variables in a social skills assessment, but cite evidence (Linehan et al., 1979; Hammen et al., 1980) that social skills training produces cognitive improvement. This is explained by reference to Bandura (1977a):

"The apparent divergence of theory and practice can be reconciled by postulating that cognitive processes mediate change but that cognitive events are induced and altered most readily by experience of mastery arising from effective performance." (p 191).

In this formulation, social skills training is conceptualised as a primarily behavioural intervention which produces an improvement in social functioning at the behavioural level through "cognitive mediation". It may be that Curran's position is not so far from that of Yardley as might at first sight appear, since both postulate a cognitive process underlying any change that may occur. 


\section{v) Social competence vs social skill - the McFall reformulation}

Fourthly, there is the difficult question of the relationship between the terms "social competence" and "social skill". Hops (1983) complains that "As the social skill literature escalates, so do the number and variety of behaviours called social skills". McFall (1982) argues that neither the trait model nor the molecular/behavioural model can provide an adequate formulation of social skill, and advocates the use of the term social competence to denote an individual's ability to succeed at a criterion level on a given task.

It may be useful to summarise at this point the reformulation of the social skills model offered by McFall (1982).

McFall (1982) seeks a way to avoid the "Scylla" of the "trait model" on the one hand and the "Charybdis" of the "molecular model" on the other. His observation is that behaviour therapists in assessing social skills pay lip service to the molecular model (roughly speaking that advocated in section iii on situation specificity) but are covertly still influenced by the trait model (i.e. the notion that social skill is a stable, measurable aspect of personality).

The trait model assumes an underlying personality characteristic, consistent across times and situations. The inference would be that social skills would be amenable to psychometric measurement. However, the results of psychometric investigation are disappointing. The various measures appear to correlate poorly with each other, and fail to predict "real life" performance. 
The molecular model begs the question "So what?" If social behaviour is specific to the situation, it is hard to see what is the value of measuring it, and what predictions may be made from it. A further problem arises when a criterion is sought for the supposed effectiveness or otherwise of behaviour " $x$ " in situation " $y$ ". At this point the social skill evaluator must fall back on subjective judgement or enlist an "expert" to comment on the behaviour in question.

To escape the "horns of the dilemma", McFall reformulates the social skills model by sharpening the definition of (a) social competence (b) social skills (c) the relationship between the two concepts.

Social competence in the proposed reformulation refers to the adequacy of performance on a given task. The person making the evaluation must be specified and ihe criterion for adequate performance made explicit.

Social skills are conctptualised as the abilities required for successful performance at a given task (i.e that on which soc $\lrcorner a l$ competence is being judged). Each of the skills must be present in order for successful performance of the task to be carried out.

A task is defined as a hierarchically organised behavioural program which organises and directs performance. In order to evaluate performance, it is suggested that task analysis be carried out. Task analysis might include examination of the purpose of the task, the prevailing constraints, the setting (which may include persons), and the social rules in operation. Adopting a "systems perspective" is also advocated in the approach to the analysis of the task. The notion entailed here is that of viewing somewhat more broadly the relationship of the task to the individual's deployment of 
resources to meet the requirements of his life situation.

To assess an individual, it is proposed that the main life task (for that individual at that time) is established; task analyses are then carried out. (The "main task" would be broken up into "sub-tasks"). The next step would be to obtain samples of behaviour from the individual in question, using real life tasks if possible. The "social competence" assessment could then be carried out on each task, using perhaps a simple "OK / not OK" criterion. Results could be summed up in profile form.

Implications of the assessment might be examined using a systems perspective. Prior to deciding on a programme of social skill enhancement, it is suggested that the expectations being made of the individual (or, more broadly, the "person/environment match") be assessed.

The social skills assessment may be carried out if the social competence assessment identifies deficits in task performance. Social skills are assumed to be those abilities that are necessary for performance of the specified task. It is supposed that they will be relatively stable across time and situations. It is assumed that successful performance will require three main types of social skill - cognitive, physiological le.g control of arousal) and overt behavioural skill.

The Argyle (1967) model is refurbished by McFall (1982) to provide a sequence of processes, grouped (as in the Argyle model) within three main sub-systems. 
The input to the system comes from the individual's prior history, and the stimulus situation or task.

In the first phase of each "cycle" of operation, the individual's decoding skills come into play; these include reception, perception and interpretation.

In the second phase (Argyle's "translation" phase) decision skills come into play. These include response search, response test, response solution, repertoire search, and utility evaluation. The individual has the task of identifying an appropriate response, identifying a suitable behaviour in his personal repertoire, and checking the value of executing that bit of behaviour.

In the third phase (corresponding to Argyle's "effector processes") encoding skills are involved. These entail execution and self-monitoring. since social skills are essentially a form of communication (either verbal or non-verbal) and the essence of the performer's task is to convey a clear message, the use of the term "encoding" is an apt one.

The outcome of the three main processes is the response; the sum of the responses on a given task is the performance. This the observer must judge to be either competent or incompetent, "O.K" or "not $0 . \mathrm{K}$ ".

As in the Argyle model, cognitive structures are integrated within the skills framework. Therefore, a social-cognitive deficit would be termed a 
social skill deficit. The McFall model goes beyond the Argyle model in making this definitive assertion.

In assessment, then, the skills required for competent performance are first identified; they are then measured in specific individuals. The usual aim is to identify deficits. Isolation strategies may be used to identify the problem area. In a similar way to the establishment of baseline on a psychometric test, if the individual can attain the "response test" stage successfully, he may be presumed to have mastered the skills required at preceding stages of the process. To test ability in the encoding process, the individual may be presented with a behaviour which has already been correctly developed to that stage and simply requires enactment. To save time and effort, common faults can be checked at an early stage in the assessment.

Intervention would aim either to modify the task to meet the individual's limited capabilities, or to provide focussed work to overcome specific individual deficits.

From the foregoing explication it may be seen that the model offered by McFall (1982) as a reformulation of the social skills model has a number of advantages. The model goes some way towards dealing with the areas of difficulty which have been discussed in preceding sections, namely empirical definition, situational specificity, and "internal elements". The sharpening of the definition of social competence and social skill helps with difficulty in offering empirical definitions and deals with the issue of situational specificity. By emphasising the inclusion of cognitive elements in social skill, McFall (1982) addresses the issue of "internal elements". The clear implication is that both cognitive and behavioural elements of social skill are important, and therefore require assessment. 
Hops (1983) suggests that we follow McFall in assuming that specific identifiable skills form the basis for socially competent behaviour and that interpersonal difficulties may arise as a function of a faulty behavioural repertoire. In relation to children, Hops notes that the social observers in the natural environment - parents, teachers and peers provide the best evidence of social competence. Peer ratings have high predictive validity and this is discussed further in section Three. Peers, teachers and parents do not always agree however (Gresham, 1981). Therefore, a global measure of social competence must be derived from the mean of a number of constituent variables. Assessment, then must be "multimodal".

Hops goes on to review a number of studies which explore the notion that social skill (observable behaviour) is functionally related to social competence (sociometric status). ( see section 5, Assessment, on sociometric ratings). The point being stressed here is that a clear distinction needs to be made between the two concepts. The repertoire of social behaviours hypothesised by Rinn and Markle (1979), based on the Hops/McFall formulation, is to be defined as one of the substrates of the construct, "social competence". Skill in social perception and cognition also contribute to social competence, since they enable individuals to deploy the right behaviour in the right social situation in order to attain the right outcome. Trower's generative social skill, briefly described above (Trower, 1984) would also provide an input to "social competence". 
In conclusion, it is important to be aware that in assessing an individual for social skills training, it may not be an easy matter to identify those elements which require remediation. It will be necessary to assess the situations in which social skills difficulty occurs and the interaction between the individual and those situations. There must be awareness of the importance of process variables - either cognitive or emotional mediating factors. Both social skill - the abilities required for performance in a particular situation - and social competence will require assessment.

\section{Areas of Application}

Social skills training has been applied very widely. Hollin and Trower and their contributors (1986) have documented the use of the technique with acute and chronic adult clients, mentally handicapped and organically impaired clients, adult offenders, the elderly, substance abusers, anorexics and transsexuals. Social skills training has also been deployed in the training of a number of professional groups and in community settings. In view of what may be called the "proliferation" of social skills training it is essential to maintain a professional standard of assessment and ensure that interventions are appropriate and soundly based on research (Hollin and Trower, 1986). Otherwise there is a danger that in the absence of clear conceptual definition social skills training will, as a result of indiscriminate use, first degenerate to a "fad" and then be replaced by the next fashionable procedure, and so vanish (Hudson, 1986). 


\section{College students.}

Owing to their availability, college students are frequently used in research on assertiveness, social competence and social skills. Jones et al. (1982) explored the link between social skills deficits and loneliness. Galassi et al. (1981) identified three causes for lack of assertiveness conditioned anxilety, lack of social skill, and "cognitive deficit". Bonner and Rich (1988) provided more detailed information of how cognitive deficit may affect mental state. Administering a number of rating scales to 186 subjects, they found that "social problem-solving self appraisal" and it's interaction with negative life stress both predicted a sense of suicidal hopelessness. In interpreting their findings, they felt that the "perceived existence of a possible solution" could be the operating factor in determining whether a sense of hopelessness was experienced. Social skills training should therefore aim to help clients to feel that solutions to social problems are available.

Furnham (1986) reports on a number of studies which explore the difficulties of young people in interacting with the opposite sex. Twentyman et al. (1981) studied "heterosocial" (i.e., dating) avoidance in college males. Non-daters were characterised by the avoidance of interactions, by low self-ratings of skill, confidence, and approach behaviours, and by having less knowledge about social cues than their more actively dating counterparts.

From the three studies here briefly summarised, it would appear that social skill (including perception of social cues) is important for at least one social activity which is of developmental relevance for this age, namely dating. Social knowledge, social problem-solving ability and social 
confidence are all important. We may postulate that "social anxiety" or "learned helplessness" operates as an intervening variable to make it less likely that further interactions will be attempted after an interaction which is perceived as a "failure". We may further speculate that selfesteem is a key "person variable" in determining the valence of this intervening variable.

There is documentation of the need to intervene with this particular problem with this client group. If left untreated, the outcome of the problem may be adverse. Philips and zigler (1964) found that failure to establish satisfactory relations with the opposite sex predicted psychological difficulty in adulthood.

There is suggestive evidence that interaction training may be of assistance to this client group with this problem. Pendleton et al., (1976) report on a programme of social interaction training using systematic homework. Subjects were "highly anxious, low frequency daters who wished to increase their dating frequency and their comfort in interactions with the opposite sex". Sessions included relaxation, skill training, cognitive control, and a homework assignment. The coeducational nature of the programme was thought to be helpful. Preliminary data showed evidence of significant change on self-report measures of self-evaluative anxiety

There is therefore at least limited evidence that college and university students may benefit from a similar type of social skills training input to that which has been developed for clinical populations. Certainly the nature of the problem is similar that which presents in adult populations when allowance is made for developmental needs. 
The finding that social skills intervention may be of assistance to college students who have dating problems is of relevance to the present study. The high incidence of sexual activity in young people below the age of sixteen implies that dating must be an issue for many in this age group. However young people develop at different rates and the approach in counselling young people below the age of sixteen may be different to that considered appropriate for college and university students.

\section{Adolescents (excluding offenders).}

In comparison with the literature on other client groups, the findings on non-offending adolescents are relatively sparse (Furnham, 1986). Nevertheless there is information available on the following areas: assertion training, job interview preparation, treatment of aggressive adolescents, and treatment of shy adolescents.

There is limited evidence for the effectiveness of assertion training. Buell and Snyder (1981), using assertion training with youngsters ranging from 8 to 18 years, found improvement on a role-play test but not on selfreport measures. Pentz (1981) also found only limited improvement on an assertion training programme. Her results indicated that individual difference variables, particularly verbal reasoning and state anxiety accounted for substantially more variance than training variables. 
Social skills training has been found useful in training young people for job interviews (Hood et al., 1982; Heimberg et al., 1982). Hood et al. found that modelling, coaching, role-play, feedback and discussion were helpful in developing verbal and non-verbal interview skills. The effects generalised and were maintained over time. Heimberg et al. found that trained subjects did better than controls both on specific role-play measures and on assessments by independent observers.

For aggressive adolescents, a successful in-school social skills programme was devised by Filipczak et al. (1980) who used a programme of instruction, modelling, role-play and feedback to change the behaviour of aggressive, unco-operative, underachieving pupils. They report significant change in school work, rule-following and courtesy in their treatment group in this controlled study, and add: "Results point to positive outcomes on multiple reliable indices of social skill across several programme years". Clearly if social skills training can be used to reduce disruptiveness in school, this is extremely useful.

Studies on "shy", anxious or socially inept adolescents are few in number particularly if we restrict the age range to 12-16. Two examples, both involving group work with adolescents of mixed gender, may be mentioned here. Lindsay et al. (1979) report on an interesting and clinically useful programme, and provide an evaluation with some case examples. In a more elaborate study, Jackson and Marzillier (1983) treated adolescents referred with social difficulties by means of a social skills training package within a Youth club context, and noted signs of clinical improvement. 16 subjects were assigned randomly to a treatment group and a waiting list control group (the latter received treatment after three 
months). The treatment group were involved - in discussion, instruction, modelling, behavioural roleplay, practice feedback, and social reinforcement. Each member received training in a target problem. Early sessions concentrated on basic social skills such as eye contact, facial expression and voice intonation while later sessions were concerned with more complex skills including conversational skills, assertive skills, and dealing with teasing. All subjects reported fewer problems from pretest to posttest on a problem checklist, but the difference between the two groups failed to reach statistical significance. This may have been due to the small size of the two groups. A significant interaction effect (i.e clear difference between the groups) was found on two sub-items of a conversational skills test. Follow-up information was somewhat disappointing and the authors suggested that "More is needed to produce long-term benefit in terms of increased social integration" (p. 318.)

This brief review suggests that while the area is under-researched social skills training may well be of considerable value for a number of nonoffending adolescent populations. It may well be ihat adolescent social failure is predictive of later psychological difficulty, but so far this has not been established.

The treatments so far described have been broadly behavioural in nature as are the majority of social skills programmes. However a less directive approach may be of value with this age group. Farrell (1980) asserts that "For adolescents, social skills are absolutely vital. Without the complex ability to merge or mesh with other adolescents, while at the same time maintaining a negotiable relationship with adults, a teenager is very handicapped" (p.2) . Farrell advocates a "playful" approach on the part of adults running social skills groups for teenagers, and echoes Yardley in 
stating that the most important task for the adult is to drop into the background and enable the teenagers to learn from each other. This is rather far from the "active, directive" model of social skills training but is nevertheless an important insight.

\section{Adolescent offenders (delinquents).}

There is a substantial literature on work with adolescent offenders, well reviewed by Henderson and Hollin (1986). There is limited evidence that both male and female delinquent populations may suffer from deficits in social skills/social competence (Freedman et al., 1978; Spence, 1981; Gaffney and McFall, 1981); however it is not established that such deficits may be a determinant of delinquency.

Contrary evidence is reported by Renwick and Emler (1991). These authors assessed 37 young male offenders using a self-report social situation inventory and a social interaction test. In the social interaction test a videotaped sequence was rated for specific skizl elements (e.g. eye contact) and for general impression conveyed. On the self-report test the delinquents scored at a similar level to the (presumably non-delinquent) university students for whom means had previously been obtained (Bryant and Trower, 1974). On the social interaction test, median ratings were reported with no major deviations. No non-delinquents were directly involved in this study. 
As delinquency implies a propensity to commit antisocial acts, it is tempting to suppose that social skills training may offer a "cure." To assess this empirically, we must first ask whether it will prevent recidivism. Clearly, we would need reliable information across an individual's life span to be sure of this, but short-term indicators may be of some value. Henderson and Hollin review 20 studies in which social skills training was undertaken with delinquents. Of these, the following three actually used recidivism as a measure of outcome of social skills training.

Spence and Marzillier (1981) ran groups with 32 male offenders aged 10-16. Using three-month follow-up of recidivism as a measure, no significant difference was found between the treatment group and delinquent controls.

Hazel et al. (1981) reported on work with a mixed group aged 13-17. Using behavioural assessment of videotaped roleplay as a measure, it was found that skills were increased during the training period. Using 12-month follow-up for recidivism as a measure, it was found that the treatment group showed less recidivism than controls. However, no statistical analysis is reported, so we do not know whether the finding was significant.

McGurk and Newell (1981) treated a sole male delinquent client in a group with two "non-trainees". This client was helped with his ability to talk with women. He showed an improvement in behavioural assessment, a decrease in self-reported social difficulty, and no reconviction at 12-month followup. In relation to this study Henderson and Hollin comment that the source of data is uncertain. 
There are three other studies which were included by Henderson and Hollin because the form of treatment was held to be analogous to social skills training. Alexander and Parsons (1973) carried out treatment with 46 subjects of mixed gender. Working with the family as a unit, they improved family interaction and reduced recidivism for their behavioural training group. Gross et al. (1980) trained 10 subjects of mixed gender in selfmanagement; the subjects increased their self-control, reduced their problem ratings, had fewer school absences, and no arrests after 12 months. Chandler (1973) worked with 15 boys on their "social egocentrism". They duly reduced their scores on this variable, whilst their controls did not, and their recidivism was less than that of the delinquent controls. Henderson and Hollin point out that the studies which appeared most effective were those in which the young offenders were not in fact incarcerated.

In a slightly different tradition, Guerra and slaby (1990) using a socialcognitive development model of treatment with 120 incarcerated male and female adolescents, found significant reductions in aggression as measured by staff rating, but no significant effect on parole violation in the control group comparison. This finding is congruent with those reported by Henderson and Hollin.

A number of researchers have explored the more long-term effects of social skills training on young offenders. Hollin and Henderson (1984) review the empirical evidence on this topic and conclude that social skills training has only a very limited effect on criminal behaviour. It is argued that the use of criminal behaviour as an outcome measure invokes the notion of response generalisation (Craighead et al., 1986) which is dubious as a 
behavioural measure of outcome (Lindsay and Stoffelmayr, 1982). Further, it may be unwise to seek to modify the individual's behaviour in this way ("first order change") without also modifying some wider system ("second order change") (Emery and Marholin, 1977; Watzlawick, Weakland and Fisch, 1974). Emery and Marholin implicate another factor in the lack of evidence for generalisation in delinquency studies; they comment on the relative paucity of longitudinal data which may be due to limited resources and the career needs of researchers for early publication. The danger of simply monitoring official recidivism rates is further high-lighted by the finding of Spence and Marzillier (1981) that at follow-up their social skills training group had the lowest number of offical convictions, but the highest number of self-reported offences.

It is necessary then to be cautious in assuming that social skills training can "cure" delinquency. Nevertheless the studies reported by Henderson and Hollin do show that social skills training may be helpful to delingents - a more modest claim. The evidence would seem to warrant the view that focused studies with a specific aim are most likely to be successful.

\section{Pre-adolescent children (ages 8-13)}

There is a substantial literature on social skills training with preadolescents. Extensive reviews on social skills training with children have been made by van Hasselt et al. (1979) and Beck and Forehand (1984). 
Van Hasselt et al. (1979) note that social isolation may be defined in one of two ways - in terms of peer acceptance, or in terms of observed frequency of interaction. They recommend a system of analysis based on a combination of the two approaches. They also express concern that while the immediate effects of social skills training may be impressive, there are limited data concerning generalisation and the maintenance of treatment effects.

Beck and Forehand identify four approaches to social skills training in children. The first, peer mediated training, uses one or more peers as a model or "assistant" to the trainer. The second, adult contingent attention, uses adults as assistants to the trainer; the adults provide reinforcement, through their attention, for appropriate social strategies. The third, modelling, makes extensive use of video material to provide information to clients about social behaviours which they might wish to emulate. The fourth, treatment packages "Denote the application of several direct intervention strategies, typically including instruction, coaching, role-playing, rehearsal and feedback" (p. 24). Whilst they consider that there is no firm research evidence as to the relative efficacy of the four methods, they find the peer mediated approach particularly attractive, in that it is inexpensive of resources and can be extremely effective. However they consider that on intuitive grounds the treatment package might be considered the most appropriate for children who are more seriously socially disabled. In their view the treatment package approach is only suitable for children who are of at least school age, as younger children might have too limited a concentration span to benefit from the programme. With older children, the effectiveness of the treatment package has been clearly demonstrated. 
Three studies will be briefly summarised, by. way of illustration. These summaries will give some indication the treatment methods used, assessment procedures adopted and outcomes obtained with preadolescent clients.

Oden and Asher (1977) conducted an intervention in school with socially isolated third and fourth grade children (9/10 year olds). Clients were selected by roster-and-rating sociometric questionnaires, in which children were asked two questions: "How much do you like to work with this person in school" and "How much do you like to play with this person in school". Children were assigned randomly to a coaching group, a peer pairing group, and a control group who received extra attention but did not interact with peers. The treatment group $(\mathrm{n}=10)$ were given coaching on five occasions. This coaching was done on an individual basis. The children received practical advice on concepts such as participation and communication and the coaching session was immediately followed by a play session with a socially competent peer, in which the child was specifically asked to try out some of the ideas introduced in the coaching session. The play session was followed by a review session with the "coach". Outcome was assessed by a repeated measures design based on the sociometric questionnaire and a behavioural assessment of play behaviour in which task orientation and social orientation were coded.

On the "Play sociometric questionnaire" (see above) the "coaching" (or treatment) group showed significantly more change than did the "peer pairing" (or placebo) group and the non-interactive controls. There was no significant change on the behavioural measures, or the "Work sociometric questionnaire". At one year follow-up there was some indication that sociometric gains were maintained in the treatment group, though the movement of children to different classes necessitated a rather elaborate 
procedure of measurement. The authors concluded that there was a clear difference between choice of work partner and choice of playmate for this group of subjects, and that the treatment group had indeed secured greater group acceptance. This they believed was due to their social skills which were enhanced by coaching, immediate exposure to a social learning task, and feedback. The authors took the view that no change was shown on the behavioural tasks because the analysis (i.e the behavioural coding system) was not sufficiently fine-grained.

This study is of interest owing to its well-designed, focused treatment approach, its employment of both peer acceptance measures and behavioural measures, and its positive outcome - including the valiant attempt at obtaining follow-up data.

Bornstein et al. (1977) had 12 unassertive children defined as "passive, shy and conforming" referred by teachers at the university school. of these, four were selected who appeared deficient in at least three "microskills" (eg poor eye contact, short speech duration, inaudible responses and inability to make requests) during baseline assessment. Jane (8) Tom (8) Mary (10) and Alice (11) all had trouble in standing up for their rights with their peers and in expressing anger when appropriate. The Behavioural Assertiveness Test for Children was used as the dependent measure. This consists of a sequence of nine role-play tasks with undergraduate research assistants used as stooge or "role model". The roleplay tasks took the following form: 
Female Model

1. Narrator (behind screen): You're part of a small group in science class. Your group is trying to come up with an idea for a project to present to the class. You start to give your idea when Amy begins to tell hers also. Prompt: "Hey, listen to my idea".

The role play behaviour was videotaped and rated for verbal and nonverbal components of assertive behaviour and for overall assertiveness. All subjects received nine sessions of SST over 3 weeks using a multiple baseline design. Throughout training six of the roleplay scenes served as training scenes and the remaining three were used to assess generalisation within training. Modelling (demonstrating appropriate behaviour in a roleplay context) and behaviour rehearsal (skills practice in a role-play context) were the principal training techniques.

The results were dealt with by multiple baseline analysis, in which the frequency of "target behaviours" is plotted in each session. The results demonstrate that as each new behaviour was trained, there was a corresponding increase in it's frequency in the role-play sessions. In this approach, there is no statistical analysis; each subject is treated as a single case design.

The results indicated that the approach produced considerable improvement in both component behaviours and overall assertiveness for all four subjects. Treatment effectively enhanced performance in verbal and nonverbal components of assertiveness that had been identified as deficient leye contact, loudness of speech, speech duration, and requests for new 
behariour). Changes from baseline levels occurred only when trainina was directed to a specific behaviour. In all cases qains obtained on treated scenes qeneralised to untreated scenes. All improyements were mantained on one-month follow-up.

The authors conclude that they have shown the possibility of identifyina specific deficzencias in verbal and non-verbal components of assertiveness in children described as lacking in social skills. They also claims that it provides support for the effectiveness of the treatment package instructions, feedback, behavioural rehearsal, and modelling. They recoonise however that there is no evidence in the study for qeneralisation to interactions with other children in the natural environment.

This study is interesting in three ways. Firstly, it applies the concept of assertiveness to children. The authors define assertiveness as the "ability to express both positive and neative feelings in the interpersonal context without sufferina consequent loss of social reinforcement." They add that "Children deficient in this reqard would require training to increase their ability to stand up for their rights and express both anger and positive feelings such as appreciation" (p. 184). Secondly this is a good example of a behavioural approach in action. Thirdly, it is an example of the multiple baseline design, frequently used in behavioural treatments.

Verduyn et al. (1990) ran a programme in an oxford Middle School with children aqed 10-13. The Rutter B2 Scale (Rutter, 1967), a measure of behavioural difficulty. and a sociometric questionnaire (Maclillan et al., 1980) were used to screen the children for social skills deficits. Cutoffs were established such that $9.3 \%$ of the children were selected. 
Subjects were allocated at random to treatment $(\mathrm{n}=17$ ) and control ( $\mathrm{n}=$ 17) groups. The intervention included 8 group sessions followed by 4 booster sessions. Clients were subdivided into 4 qroups. Sessions followed a formal structure comprising Homework Feedback. Harm Up. Introduction to Theme (themes include coping with bullying, responding to criticism, giving compliments), Didactic Period, Behavioural Rehearsal, and Homework Setting. Dependent measures included a social behaviour checklist completed by parents and teachers, a social situation checklist (Spence, 1980), a selfesteem inventory (Coopersmith, 1967) a diary of social activities, and a clinical chanqe ratina. On the Parents Behaviour Checklist, a significant reduction in problem behaviours was found post treatment and at six month follow-up Analysis of covariance indicated that the reduction in problem behaviours in the treatment aroup was due to the intervention. Posttreatment, the aroup receiving social skills training were more socially active than the controls and had themselves made significant improvements in their social activity: these results were maintained on follow-up.

This study is of interest firstly in that the treatment package is delivered to children in small groups; secondly because the treatment is dalivered in school - a natural setting, where generalisation may readily be monitored: thirdly, because it uses a wide range of assessment measures.

These three studies are reasonably representative of the wide range of work on social skills training with preadolescent children reported in the literature. It may be noted that each uses some form of treatment package, and each claims to provide some evidence of effectiveness for the method. In the third study, a group trainina approach is used while the first and second employ child and adult models respectively as "aides" in the training. 


\section{Younger children (0-7).}

Beck and Forehand (1984), as previously stated, identify four treatment approaches: adult contingent attention, peer-mediated strategies, modelling, and treatment packages. of these, it would appear that the first three have been used most successfully with younger children.

In adult contingent attention, the adult "shapes" a child by attending when a child produces appropriate interactive behaviours (Walker et al., 1979). In peer-mediated strategies, a peer may be taught social skills and then be asked to teach an isolated child "how to play"; or play sessions may simply be programmed so that isolated children have an opportunity to interact with a non-isolated peer (Furman et al., 1980). In modelling, children may be shown a video of a child gradually joining a group of peers in a play situation (0'Connor, 1969). In a clinical case study, a six-year-old was involved in participant modelling with an adult model/therapist, and induced to play with a group of unfamiliar children by a method of gradual exposure "in vivo" (Ross et al., 1971). Beck and Forehand report only one case of a treatment package used with preschool age clients. Combs and Lahey (1981) used a treatment package which consisted of self-instructional training, modeling, and reinforcement. They failed to find any changes in eye contact, peer contact, verbal responses, and verbal initiations of three pre-school children who were recommended by teachers as being unskilled and were observed to be socially withdrawn. In this study the assessment and intervention were carried out in different settings.

The above techniques may be enhanced by "coaching" (Oden and Asher, 1977) and in this context the teacher often becomes the trainer/therapist. It may 
prove difficult for teachers to acquire appropriate skills. Frosh (1983) suggests the methods of continuous commentary which involves maintaining a flow of comment about appropriate social interactions, and problem-solving whereby the teacher enacts and models, within the classroom, procedures for dealing with interpersonal difficulties as they arise.

\section{Conclusion.}

cleariy, in providing social skills training to children and adolescents, allowance must be made for developmental need. older children seem to respond well to the more complex treatment packages; younger children require a more immediate approach. older adolescents may need guidance on special areas such as dating. However, a common thread runs through the age groups; there is evidence that an active, directive approach to social skills training can help children and young people to overcome difficulties in peer interaction and achieve peer acceptance. 


\section{A Developmental Approach to Social Skills Training}

\section{Rationale for social skills training.}

There are sound empirical grounds for undertaking social skills training in particular with children and young people. Van Hasselt et al., (1979) review fourteen studies linking childhood social difficulties to later problems of adjustment. There is in their view "A considerable body of research demonstrating a relationship between children's level of social functioning and their long-term adjustment." (p. 413) Social isolation in childhood is associated with juvenile delinquency (Roff et al., 1972), dropping out of school (Ullmann, 1957) bad-conduct discharges from the army (Roff, 1961) and mental health problems (Cowan et al., 1973).

We cannot assume that lack of social skills is the sole cause of poor social functioning; nevertheless, there is evidence that remediation of social skills improves social functioning. It has been established in the preceding section that social skills training is not a panacea for delinquency; however, there is evidence from at least two studies that social skills training can help disadvantaged young people to avoid delinquency and related difficulties. Sarason (1978) studied institutionalised boys between the ages of 15 and 18 who had committed serious offences. In 16 1-hour group sessions, models demonstrated specified behaviours - resisting peer pressure, asking for help, and dealing with authority figures. The boys then rehearsed the behaviours. In addition to significant short-term effects (staff ratings, self-reports, and behavioral variables), the recidivism rate was significantly lower for the group that received social skills training than for a comparable control group. Five years after the programme, the group that received social skills training showed a 50\% reduction in recidivism. Their 
reduction in recidivism was greater (but not significantly greater) than that achieved by a discussion (placebo) group.

Sarason and Sarason (1981) carried out a study with 127 low-achieving students of mixed gender attending an urban multi-ethnic high school which had a high rate of dropout and delinquency. The mean age was 14.8 years. Again modelling (live and video) and role-play were used. The subjects saw demonstrations of both effective overt responses (e.g. how to ask a teacher a question) and the cognitive antecedents of effective behaviour (e.g. deciding between alternative courses of action). Areas emphasised included consequences of an action, effects of an individual's behaviour on others, increased understanding of another's point of view, and communication skills. Cognitive material was incorporated in the modelling scripts as shown in this example:

"Jim's cognitions: Gee, I really want to go to that party. Maybe I can get up the nerve to ask Lydia out. But I should stay for that algebra review. at least. The test will be hard enough without missing the review."

There was evidence of improvement in social-cognitive skills in that subjects who received social-cognitive training were able to think of more and often better alternatives to problematic situations than did controls. Trained subjects did significantly better than untrained controls in job interviews held a few months after completion of the training. In terms of lateness for class, absence, and referrals for behaviour difficulty, the 
trained students had a significantly better outcome than untrained students over the year following the training.

The authors felt the results were encouraging. They note that a structured package of this nature, with videotaped material and exercises, may be administered by committed teachers without psychological expertise; therefore the package is cost effective. They believe that the programme may have generalised results in preventing maladaptive behaviour.

The findings provide limited evidence of the potential of social skills training to enhance the social functioning of low-achieving young people in disadvantaged settings. In view of the research evidence previously cited, it is likely that young people helped by such programmes will benefit in terms of their later adjustment to a variety of life situations. Sarason and Sarason state: "It seems clear, not just from observation, but from a wide variety of research data, that interpersonal skills are associated with adjustment throughout life" (pp 916-7). It seems reasonable to infer that in addition to these adolescents, younger children might derive similar long-term benefits from appropriate training in social and interpersonal skills.

Antecedents of social skills difficulty.

In this section, consideration is given to possible determinants or risk factors for social skills difficulty. 
Following the social learning model, Curran (1979) attributes poor social skills performance to five factors, namely: i) failure to acquire the relevant skill; ii) the presence of less appropriate behaviours owing to faulty reinforcement; iii) interference by emotional states/prior excessive negative reinforcement; iv) poor social perception; v) faulty cognitive assumptions.

Referring to Curran's first factor - failure to acquire relevant skill Herbert (1986) emphasises that in order for friendships to develop there must be opportunities for children to come into contact with each other and interact. The absence of this possibility, then, would be a possible proximal antecedent. An example of faulty reinforcement of less desirable behaviours would be "coercion" (Patterson and Reid, 1970). Children may have learned to influence peers and others through punishment and negative reinforcement. Rinn and Markle (1979) term this "control by pain", and suggest that within peer interaction, this tendency in an individual may ultimately lead to rejection.

Some children find it difficult to initiate social interactions. This may be because of emotional interference (eg excessive anxiety conditioned by prior experience) or faulty cognitive assumptions (eg "They will be horrible to me and I will get upset"). If, as a result of early experience in the social environment, a child develops an undue degree of social anxiety or very negative expectatios of social interaction, a possible consequence is reduced self-esteem. This, in turn, may inhibit social interaction. Self-esteem has been investigated by Coopersmith (1967). He found that in order to achieve high self-esteem, a child needs maximum autonomy within firm limits at home together with stable social norms outside the home. Such children frequently initiate interactions and 
show little anxiety.

Herbert (1986) further comments that lack of consistent feedback in infancy (Seligman, 1975) and insufficient experience of mastery of the environment (White, 1959) are likely to "interfere with a child's sense of personal adequacy and hinder the acquisition of skills" (p 23). Seligman (1975) suggests: "At some primitive level, the infant calculates the correlation between response and outcome. If the correlation is zero, helplessness develops" (p. 139). White (1959) has developed the notion of "effectance motivation". He refers to the need to deal effectively with the environment. From his research he reports that even very young babies show evidence of this type of motivation.

Obviously, social competence is not the only determinant of status among peers in children. Other factors, such as physical attractiveness or academic achievement (Green et al., 1980) may be involved in social status.

\section{Characteristics of children requiring intervention.}

Curran (1979) emphasises the importance of grounding both behaviours to be trained and the situations used in training on an empirical basis for a particular subject population. The social skills trainer, in Curran's view, requires extremely clear and detailed information regarding the particular skill to be developed and the particular situations in which that skill is to be applied. As an example of an empirical approach to this task, he cites a study reported by cox et al. (1976). These investigators generated items of social interaction on videotape, after collecting verbal and nonverbal material by means of a literature search. A contrasted groups design was then used to determine which items of videotaped interaction 
discriminated between problematic and non-problematic children. This enabled the investigators to generate material that was suitable for a client group with which they were unfamiliar.

Beck and Forehand (1984) report that friendly behaviour towards peers (Hartup et al., 1967) is characteristic of children with high sociometric status while children with low status are likely to have limited knowledge of how to make friends and to be poor in communication skills (Gottman et al., 1975). Consequently children lacking in these respects may require help.

Coie and Kupersmidt (1983) compared the social status and behaviour of boys aged 9-11 in newly constituted groups with their previous status and behaviour in school. They found that rejected (aggressive and disruptive) boys tended to reproduce their behaviour in the unfamiliar groups more than did neglected (shy and withdrawn) children, providing support for the finding of Asher et al (1981) who suggested that rejected and not neglected children were at risk for future problems. Exploring behavioural differences between "rejected", "popular" and "neglected" children they noticed that in the new situation the "rejected" children were active and aversive, the "popular" children were norm-setting and prosocial, while the "neglected" children were least interactive and aversive but more visble and active than the other two groups. The authors comment on the importance of distinguishing behaviour associated with the emergence of social status from behaviour associated the maintenance of social status. 
Dodge (1983) using video recording analysed the behavioural correlates of social status in 7-8 year old boys who were previously unacquainted with each other. Predictably, boys previously classified as "rejected" on grounds of "antisocial" behaviour showed excessive amounts of physical aggression. More interestingly, there was no problem of initial interaction in either the "neglected" (withdrawn) boys or the "rejected" (antisocial) boys. Where they came "unstuck" was in their inappropriate social approach. It appeared that the popular children in the study had skills in maintaining interaction. It seemed likely that the rejected and neglected boys became isolated in the later sessions as a result of negative interactions in the earlier sessions. Popular (high status) boys engaged in high rates of cooperative play and social conversation. Dodge attributes the low status of the neglected and rejected boys to inept peer interaction (together with antinnaial behaviour in the case of the rejected boys).

Putallaz (1983) de:ised in ingenious study in which 22 young boys, individually, were introduced to two somewhit clder unfamiliar peers who were confederates in the experiment. Each boy played games with tha $t$. $C$ "stooges" for a while in accordance with the experimental procedure. This behaviour was videotaped and coded. Four months later, on entry to elementary school, sociometric procedures were carried out to determine the social status of the 22 boys (who were placed in various different classes). The tendency of subjects to fit into the group they entered by contributing relevant conversation was predictive of their social status even after controlling for intelligence. 
Asher (1983), in reviewing the above three studies, concludes that a causal relation between social behaviour and social status is established, though clearly there may also be an interactive effect. The studies need to be replicated with female groups or with groups of mixed gender. It is known that boys and girls differ somewhat in their patterns of social behaviour.

It would seem from the above studies that low sociometric status together with inept peer interaction may be indicators of the need for social skills training. There is a suggestion that rejected children, who combine inept peer interaction with antisocial behaviour, may be particularly in need.

\section{Assessment for Social Skills Training}

In planning assessment for social skills training, it is generally expected that consideration be given to the following questions: the type of difficulty; how these difficulties are to be measured; whether clients should be seen individually or in groups; if the latter, whether clients with different types of problem should be treated together or separately; what assesment measures are to be used; and whether these provide information about related or unrelated variables. For example, in child studies rather different sets of results may be obtained when both sociometric and behavioural measures are used.

In assessing children, investigators will seek to ensure that the measures are appropriate for the child's level of development (Curran, 1979). Account needs to be taken of clinical manifestations of need which may not easily be detectable by statistical procedures (Winter and Marzillier, 1983; Trower, 1984). 


\section{Assessment of client needs.}

Curran (1979) takes the view that whatever the aetiology, social skills training is robust enough to be helpful with a wide range of difficulties, since in his view interpersonal skills, once learned, may generalise to new situations.

However, to ensure provision of appropriate treatment, it may be important to distinguish between those children who are "neglected" by the peer group and those who are "rejected" in planning assessment procedures. This is normally done by roster-rating sociometric questionnaires, in which the child is presented with an alphabetical list of classmates and asked to carry out a sociometric rating in response to one or more questions; e.g the child might be asked to rank-order class members in terms of whom he would prefer as a partner in a work or play activity. Children who fail to receive positive ratings (e.g. of friendship choice) are termed "neglected"; children who receive high negative ratings (e.g. are disliked) are termed "rejected" (Ladd and Asher, 1985).

Conger and Keane (1981) comment that "Some investigations (0'Connor, 1972) have conceptualised social skills deficits in terms of social isolation as a function of withdrawal, whereas others (Gottman et al., 1975) have focused on isolation resulting from rejection." (p. 491). Conger and Keane emphasise the need for clear definition of the different populations.

Tiffen and spence (1986) gave the same social skills training programme to "rejected" and "neglected" (or isolated) children, in an effort to identify the group benefiting most from the procedure. In the 
event, however, neither group was significantly improved by treatment. Notably, an analysis of variance indicated that across all groups in the study including the control and "attention placebo" groups, the neglected group improved more in terms of friendship nominations received than did the rejected group. This would suggest that socially rejected children are indeed more at risk of deterioration than socially isolated children. The authors conclude: "The study failed to demonstate that social skills training can produce beneficial changes in social competence as measured by sociometric status, self-reported social problems, teacher rating of social skills, and direct behavioural observation of quantity of peer interaction with children selected on the basis of isolated and rejected peer sociometric status." pp 351-2.

In conclusion, the literature provides us with information about two types of child who may require social skills training: (i) the "neglected" or isolated and (ii) the "rejected" child, who as a result of aversive social behaviour, tends to become increasingly unpopular. An empirical method for assigning children to these categories is available. As yet, there is little evidence as to the specific implications for social skills training procedures with these two groups. Intuitively, it seems likely that while both groups would benefit from training in prosocial skills le.g turntaking, conversation, listening) the isolated children are also likely to require assertiveness training such as that described in the example from Bornstein et al. (1977) whilst rejected children and adolescent would benefit from procedures designed to develop self-contrcl through cognitive means, using techniques such as those reported above in the study by Sarason and Sarason (1981). In any event, there are arguments for including sociometric ratings in the assessment procedure, as this provides valuable information about client difficulties which may inform the planning of the 
training programme.

\section{Selection for treatment.}

The criteria for selection of childrem for inclusion in social skills training programmes are widely divergent. Different "yardsticks" are used and there is little agreement as to "cut-off" or "case-ness" on any given yardstick. This is partly because a variety of techniques have been applied across different age groups, with an emphasis on applying social skills training in contexts where it seems likely to be useful, rather than starting from a basis of identified need. In the preschool programmes, aimed at early intervention, as many as $50 \%$ of the age group in a school may be involved (e. g. O'Connor, 1972). Bornstein et al. (1977), wishing to demonstrate the effectiveness of an assertiveness programme, selected children who were extremely unassertive on their own criteria and those of others. In general prevention measures are employed with younger children, whereas more extended treatment packages are conducted with older children;in their case the identification of social skills difficulty is likely to be more clear-cut, as in the study carried out by Verduyn et al.(1990). Typically, multiple measures are employed in studies of this type.

Each of these approaches is entirely legitimate in terms of the aims of the investigators. However, the implication is that there is little agreement in the literature as to the prevalence of social skills difficulty in chidren. Van Hasselt et al. (1979) reports the finding of Gronlund (1959) that $6 \%$ of the children in grades 1-3 in one school had no friend, and $12 \%$ had one friend. This finding was replicated by Hymel and Asher (1977). Consequently, it would seem reasonable to assume that some $10 \%$ of younger 
school children need some systematic form of social skills training such as a treatment package. At a speculative level, it might be argued that a proportion of the "neglected" children might improve by the time of adolescence, whereas many of the "rejected" children might deteriorate; therefore the adolescent population requiring social skills training of a comprehensive nature might be rather larger, and might include a higher proportion of the "rejected". Adolescence is, in any case, a period of increased social stress, as Jackson and Marzillier (1983) recognise. The need for social skills training may be greater in disadvantaged areas, as Sarason and Sarason (1981) point out.

\section{Types of measure.}

Trower (1982) advises that assessment should be aimed at measuring "competence" which he defines as "Social knowledge and the ability to make social plans". He further suggests that individuals' outcome expectancies should be assessed, as this provides evidence of their application of social knowledge. Mize and Ladd (1990) have applied this cognitive approach to social learning with children. They trained children in four skills; "leading" peers, asking questions of peers, making comments to peers, and supporting peers. They define the aim of their "model" as follows: "to address potential deficits in children's knowledge, the ability to translate knowledge into skilled performance, and the ability to monitor social interaction". Their training group showed a significant increase in social skills and knowledge, but no change in sociometric status.

To assess social knowledge, children were presented with three vignettes by Mize and Ladd (1990). These included a"conflict" situation in which a peer knocked down the subject's block tower, a "support" situation in which the 
subject observed one peer being teased by another, and a "potential entry" situation in which the subject observed two peers playing with blocks while other toys lay nearby, unused. Naive coders rated children's responses to each hypothetical social dilemma in terms of its friendliness and assertiveness.

Forms of cognitive assessment used with children will be considered in more detail in the next section, as they are related to a specialised form of treatment.

In the mainstream of social skills training work with children, van Hasselt et al. (1979) describe the following types of measure: sociometric questionnaire, teacher rating, interview, self-report inventories, socialcognitive tasks (for example role-taking (Feffer and Gourevitch, 1960), naturalistic observation (typically observation of social interaction rate) and role-play techniques (eg Bornstein et al., 1977) . Other authorities offer similar lists (Foster and Ritchie, 1979; Rinn and Markle, 1979; Conger and Keane, 1981; French and Tyne, 1982). Conger and Keane (1981) report six studies involving younger children in which teacher nomination is used in conjuction with social interaction rate. It is harder to carry out naturalistic observation with older children as they tend to be more aware of the observer; consequently there is a greater reliance on other methods.

\section{Critique of measures.}

There is a general problem in relation to the validity and reliability of 
the self-report measures (Spence, 1983). In the adult literature, Tyler and Tapsfield (1984) complain that the amount of information available from the literature on using self-report instruments psychometrically is scarce. With children, self-report instruments are generally of two types: firstly the social situation checklist (Spence, 1980) and secondly the child's prediction of behaviour in hypothetical social situations (Van Hasselt, 1979).

Role-play assessment is useful in planning behavioural treatment (Bornstein et al., 1977) as the training may be based on the role-play performance; however, the validity of such techniques has been called into question (Bellack ,1983; Bellack et al., 1979; Bellack et al., 1978; Van Hasselt et a1. 1981) on the grounds that changes produced in a role-play "scene" may not generalise to real life. Williamson et al. (1983) derived a set of role-play variables from a new role-play test which they had devised (the Social skills Test for Children) and comparing these to a set of criterion variables (evaluations of social competence by peers, teacher, and self).

(In previous studies, one-to-one correlations between individual measures had been computed.) Williamson et al. used the technique of canonical correlation, which explores the relationship between clusters of variables. While the highest canonical correlations were only of moderate magnitude, the authors point out that for several of the role-play variables, the correlations with the cluster of criterion variables were of greater magnitude than the simple correlations among the criterion variables themselves. In other words, they claim that there was as much variability among the other criteria themselves (assessments of competence by peers, adults and the like) as there was between certain of the role-play variables and the cluster or set of criterion variables. They therefore suggest that the role-play test may be at least as useful as the other 
measures of social competence.

Herbert (1986) cautions that social skills training researchers should ensure that the scores on assessment measures do indeed represent the upper limit on clients' social functioning (Kazdin et al. 1982, 1983). Kazdin et al. are concerned that researchers make no attempt to maximise performance to check whether a skill is in fact present. Herbert is also concerned that researchers should be aware of the possible effect of type of assessment on the behaviour displayed. Beck et al.(1980) have demonstrated that frequently the behaviour elicited can be a function of the assessment measure. They found that role-play "scenes" elicited different behaviour in children form that shown in naturalistic situations.

\section{Relation between variables.}

As reported above by Williamson et al. (1983), the correlations between the various measures of social competence - self-report, or assessment by peer/teacher/parent - are typically of low or moderate magnitude. A possible explanation is that this is because the various observers see the social behaviour of the subjects in different situations and from different psychological perspectives. The findings of Gaffney and McFall (1981) on delinquent adolescent girls are of interest in this connection. They developed a problem inventory which discriminated significantly between delinquent and non-delinquent girls. The items which did not discriminate were developed in consultation with teenagers. A possible inference would be that delinquency entails skill deficits in relating to adult authority figures. The finding suggests that children and adolescents may be judged quite differently by peers and by adults. 
Gresham (1981) using a pre-adolescent sample who had low sociometric status found high correlations between sociometric friendship nominations and sociometric rating scales, and low to moderate correlations between sociometric measures and observed behaviour (initiating positive/negative interaction, receiving positive/negative interaction). Using principal components factor analysis, they identified three factors: friendship, equating to the peer nomination measures; likeability, equating to the peer sociometric rating; and social interaction, relating to the observed behaviour measures. The Gresham study therefore provides evidence that there may be high variability among measures, particularly between behavioural and psychometric measures. The findings of Gresham are congruent with those of Coie and Kupersmidt (1983), Putallaz (1983), and Dodge (1983). Each of these studies is summarised above in the section on "Children requiring intervention".It would appear that there may be some causal effect of social interaction on sociometric status but correlations of high magnitude should not be expected. Firstly, there may be other determinants of sociometric status, and secondly, the effect of social skills training (or other learning in the natural environment) may be expected to have a more immediate effect on social interaction, and a more delayed effect on sociometric status. Sociometric status, once established in the early stages of group formation, may be rather slow to change. Ladd and Asher (1985) support this general view. If other variables enter the equation - role-play techniques, social-cognitive measures, teacher or parent ratings - the correlations are likely to be even lower, as there is likely to be more interference from other factors.

There is then high probability of variation among measurements when different forms of assessment are used in social skills training programmes 
with children. This is not a great cause for concern, as the sources of information are diverse. In considering results, it is appropriate to examine the amount of change on each variable separately, and from this analysis to arrive at an integrated view of the outcome of the investigation.

\section{Conclusion.}

It may be useful, if possible, to distinguish between "rejected" and "neglected" children in carrying out assessment. At least, it is generally considered appropriate to use sociometric procedures, though these may be somewhat elaborate. It is likely that at least $10 \%$ of a school population may require systematic social skills training. Role-play has value in training, but its validity in assessment is somewhat problematic. Most authorities favour a multimodal approach to the assessment of social skills/social competence in children. Hops (1983) following McFall (1982) has argued that some measures should sample interactive behaviour, and others should look at the assessment made of an individual's behaviour by relevant observers. If naturalistic observation is difficult, analogue or self-report measures may be used suggesting the likely response of an individual to specific social situations. A third dimension of interest is that of the so-called process variables within the individual, e.g. cognitive- social learning (Mize and Ladd, 1990; Ladd and Asher, 1985), and role-taking tests (Van Hasselt et al., 1979).

\section{Intervention}

In planning an intervention it is important to consider the developmental level of the client group and the research information available on the 
efficacy of different forms of treament. It will also be necessary to balance the relative advantages and disadvantages of group versus individual treatment packages. Other factors to be determined include the setting for the intervention and the components of treatment.

\section{Setting.}

A number of settings have been reported for social skills training with children and adolescents; school is the most common. Studies have been carried out in school by Oden and Asher (1977), Sarason and Sarason (1981), Bowdler and Gleisner (1982), and Pellegrini and Urbain (1985). other settings include universities (Bornstein et al., 1977) clinics (Rinn and Markle, 1979) and a Youth Club (Jackson and Marzillier, 1983). There is an advantage in using natural settings; it avoids stigmatisation. School has the following advantages: it is convenient for carrying out measurement, it is a major setting for the child's social interaction, and it may be possible to involve teachers both in intervention and in promoting generalisation. Within school it may be possible to gear intervention to differing levels of need (Pellegrinl and Urbain, 1985). A disadvantage of the school setting may be a reduction in direct access to parents and families. Home settings do not figure in the literature and it may be argued that there should be more involvement of families in supporting Social Skills Training.

Developmental appropriateness.

Conger and Keane (1981) assert: "It is important to uncover knowledge of the typical development of behaviour related to social competence because this may provide a framework in which deficits or deviations from the norm may be compared and ultimately corrected. The delineation of a particular 
set of skills that are important to develop at a specific age should certainly enhance the efficacy of social skills programmes" (p. 493). This raises issues concerning the proper means of establishing such knowledge. A possible approach to this task is exemplified by the study of cox et al. (1976), reported in the preceding section. It will be recalled that these authors used a contrasted groups design to discover which skill items, garnered from the literature, discriminated appropriately between skilled and unskilled children.

Developmental level may be particularly important in relation to socialcognitive abilities, of which van Hasselt et al. (1979) mention the following as relevant for social skill: discrimination and labelling of emotion, accurate and effective communication to another person, ability to take the role perspective of another on perceptual tasks, and simultaneous consideration of one's own and another's point of view. It is clear that intellectual and maturational factors will be involved in the development of each of these abilities.

Findings on social interaction with preschool children may not generalise to older age groups. Equally, it may not be possible to develop programmes with children by extrapolating from models used with adults. For example intonation and voice quality may be more important than introduction and leavetaking procedures in initiating and maintaining social interaction.

\section{Components of treatment.}

For older children, young people and adults, trainers favour a fairly comprehensive treatment package, typically including instruction, coaching, behaviour rehearsal, behaviour shaping, and homework setting, 
features which are listed by Beck and Forehand (1984). Oden and Asher (1977), in their study on preadolescent children summarised above, followed up each coaching session with a peer pairing session in which the instructions were put into practice; feedback was then given.

Mize and Ladd (1990) adopted a similar format in their social cognitive learning approach. With adolescents, it is more common for pairs of adult trainers to work with groups of adolescent peers. It is then possible to generate more elaborate role-play using the group. The group session would be very structured, with processes of instruction, role-play practice, and feedback pre-planned in some detail. 
Some authorities (Spivack and Shure, 1976; Pellegrini and Urbain, 1985) advocate a "pure cognitive" approach. Here the emphasis is heavily on cognitive training rather than behavioural training. The approach is derived from D'Zurilla and Goldfried (1971). Clients are trained in problem-solving which may involve general orientation, problem definition, generation of alternatives, decision-making, and verification. Developing this approach, Crick and Ladd (1990) have investigated outcome expectations and strategy evaluations, asking children to describe the likely outcome of strategies such as physical aggression, commands and compromise. They found that outcome expectations and strategy evaluations vary as a function of the child's status among peers. This could be interpreted in a number of ways, but one suggestion would be that social-cognitive skill is correlated with ability in social interaction, and that each of these makes a contribution to a child's status among peers.

One way to respond to data of this nature is to incorporate cognitive features in the treatment package. An example of this approach is reported by Conning and Head (1990) who ran a group of 4 buys aged $6-10$. One of their sessions is described as follows: each member gives an example of bullying, a specific example is discussed, alternative responses are "brainstormed", the most appropriate response is chosen and role-played and the consequences are evaluated. The package was effective in that the boys showed improvement both in formal measures of change and in reports from teachers and parents.

However, examination of the literature suggests that this approach may not be necessary to produce cognitive changes. In the adult field, Kagan (1984) has demonstrated that social skills training can produce improvements in social problem-solving without a cognitive component being incorporated in 
the treatment. Mersch et al., (1989) found that social skills training compared with a cognitive approach (Rational Emotive Therapy) gave similar improvements in social phobics. Stravynski et al. (1982) compared two groups of socially dysfunctional patients, one treated with social skills alone and the other treated with both social skills training and cognitive modification. Both groups improved significantly and equally.

With children (socially maladjusted boys), Michelson et al. (1983) report a comparative outcome study. Behavioural social skills training and interpersonal problem-solving produced equal improvement on a range of "social competence" measures but not on "behavioural" measures. At 12-month follow-up, the behavioural group maintained its gains but the interpersonal problem-solving group did not. A control group was used in the study but there was no interaction of time $\mathrm{x}$ condition.

Mize and Ladd (1990) using a combined cognitive and social skills package trained low-sociometric-status preschool children in interactive skills. Increase in skills was correlated with an increase in "knowledge of friendly strategies".

Further investigations are required, but the findings reported are congruent with the view of Curran (1984) that behavioural learning may produce cognitive change. Additionally, many of the components of traditional social skills training are geared to produce cognitive change anyway. Instruction and coaching are aimed at developing concepts, and the process of behaviour shaping and target setting is likely to reinforce relevant concepts at a cognitive level. 
Whilst the inclusion of explicitly cognitive components is contraversial, the case for training in social perception has been persuasively argued (Forgas, 1983; Spence, 1980). Visual stimuli (photographs or videotape) are used, together with live modelling of facial expression, gesture and posture by trainers and group members. The work of 0 'Connor on symbolic modelling ( $0^{\prime}$ Connor, 1969) underpins the value of this approach. By guided observation, the client may be helped to acquire both encoding and decoding skills simultaneously. This point applies to both visual and auditory communication.

Group treatment versus individual treatment.

Many of the early social skills training studies with children used individual treatment or peer-mediated strategies. The value of these approaches lies in the direct rapport that may be obtained and in their responsiveness to the needs of the individual.

One example of a group training procedure is reported by Lagreca and Santogrossi (1980). They selected thirty children aged 8-11 who were low on peer acceptance ratings. They used coaching, modelling and behaviour rehearsal to teach the following: smiling, greeting, joining, inviting, conversing, sharing, cooperating, complimenting and grooming. Their training group improved relative to an attention placebo and waiting list control on measures of skill knowledge, role-play assessment and initiation of social interaction. There was no improvement in sociometric status. The findings were consistent with previous research on behavioural outcomes (Bornstein et al.,1977) but not with the research of Oden and Asher (1977) which showed improvement on sociometric indices alone. 
Group training designs are sometimes favoured with older children and adolescents (Tiffen and Spence, 1986; Jackson and Marzillier, 1983; Verduyn et al., 1990; Conning and Head, 1990; Lindsey et al., 1979). Group training designs have the advantage that they enable peer modelling strategies to be used. Developmental psychologists are aware that as children approach adolescence, the significance of the adult world tends to diminish somewhat and the peer group assumes greater psychological salience (Farrell, 1980).

A finding which has some relevance for peer modelling strategies is the example of peer learning reported by strain et al. (1976), who examined the effect of prompting and social reinforcement on behaviourally handicapped preschool children, and found that there was a "spillover effect" on the social interaction of peers who never received prompting and reinforcement of positive social behaviour.

It may be inferred from this finding that in group training incidental learning from peers is liable to occur in addition to the benefit expected from the formal content of sessions. Indirectly, then, this finding provides support both for peer-modelling strategies and for group training.

Sacks and Gaylord-Ross (1989) working with visually impaired students aged 7-12 years, trained six social behaviours on a multiple baseline format, similar to that in the case example reported by Bornstein et al. (1977). The authors found that a group trained with a peer-mediated strategy showed better generalisation and maintenance of gains than did a teacherdirected group. They attributed this to the presence of non-disabled peers in both the training and natural environments. 
This is an argument both for group training and for work in natural settings. The peers will be more likely to reinforce each other in the natural environment.

There are then valid arguments for employing a group training design with young adolescents. The challenge is to incorporate sufficient attention to the individual. This issue will be addressed in section 11 .

\section{The Assessment of Outcome}

Design.

Van Hasselt (1979) reports on 20 single subject experimental designs, and nine group comparison designs. Single subject designs enable detailed analysis of behaviour change in an individual to be undertaken. An example of single subject design is the multiple baseline design (eg Bornstein et al., 1977). This enables change on specific targeted behaviours to be sequentially graphed.

Group comparison designs may be used in the following ways:

i) to establish the effectiveness of treatment by comparing levels of change in treated and untreated groups;

ii) for comparing different treatments, eg standardised vs individualised coaching (as reported by Hymel and Asher, 1977), behavioural treatment vs cognitive problem-solving (as reported by Michelson et al., 1983); 
iii) for comparing the effect of the same treatment on different groups, eg neglected vs rejected children (Tiffen and spence, 1986).

Measurement.

Rachman (1980) asserts: "There is now broad agreement that multiple objective and subjective measures of specific effects are necessary to evaluate any form of treatment" (p. 239). Beck and Forehand (1984) in reviewing 32 studies of social skills training with children found only two that did not have adequate design. 31 used behavioural observation as an outcome measure, 6 used sociometric rating, 2 used teacher rating/questionnaire, and one used a social-cognitive measure (knowledge of social skills).

More recently there has been a development of social-cognitive measures. Mize and Ladd (1990) assessed children on their response to social vignettes, as described above. Van Hasselt (1979) describes the use of another social-cognitive measure, the role-taking test. The role-taking test is similar to the social vignette technique, but specifically requires that the child predict the behaviour of another, and show ability to consider his own behaviour and that of another simultaneously.

Asher (1983) commends the three papers of Coie and Kupersmidt (1983), Dodge (1983), and Putallaz (1983) for studying social interaction over time, using fine-grained coding systems, employing multiple measures and videotaping behaviour in carefully designed analogue settings. These measures are useful for developmental research, but require considerable input of resources and may not always be practical in clinical/educational 
settings.

Broadly, then, the literature would suggest that there are four types of measurement which are most appropriate for field studies - behavioural observation, sociometric nominations and ratings, teacher ratings and questionnaires, and social-cognitive measures. Self-report measures (e.g. Spence, 1980) are also found useful particularly with older children and adolescents who are likely to be more reliable in their reporting.

\section{Client differences.}

i) Developmental level.

As indicated above, it is important to control for developmental stage, both in planning social skills programmes and in making comparisons between studies. Barrett et al. (1978) state: "Child psychotherapy research has not controlled for the developmental stage of the child. Age, corrected for maturation/developmental level, is a significant variable and must be better defined and controlled" (p. 428). This factor may account for the failure of Hymel and Asher (1977) to replicate the significant findings of Oden and Asher (1977), in that Hymel and Asher used some older children for their study. For these children, the treatment package may have been less effective. 


\section{ii) Severity of problem.}

Hops (1983) makes the point that client groups in the literature vary widely in terms of the severity of their difficulties. In some studies $50 \%$ of a given "normal" child population may be considered problematic. Findings with such a client group should not be expected to generalise to more highly selected populations. In others, cases of extreme severity may be selected (e.g. Bornstein et al., 1977). Such differences need to be recognised in making comparison between studies. If researchers can find a way to assess the severity of difficulty in children whom they train in social skills, the quality of their reporting will undoubtedly be enhanced. As has been indicated, this area is extremely problematic. However, Matson et al. (1983) have developed a rating scale to measure social skills in children - the "Messy". (Matson Inventory for the Evaluation of Social Skills in Youngsters) . A self-report form was completed on 422 children while 322 children were rated on a teacher-report measure. The age range was 4-18. Items with a test reliability of the order of .50 or greater were included in the final 62-item self-report and 64-item teacher-report scales. Using factor analysis, two major factors were identified across the two forms: appropriate social skill (e.g. "I look at people when I talk to them", "I walk up to people and start a conversation" / and inappropriate assertiveness (e.g. "I pick on people to make them angry"). Examination of the items suggests that this form would be most useful in identifying overassertive, rather than underassertive children; however the latter (i.e. neglected) children might be identified by having a low score on the "appropriate social skill" set of items. Intuitively it would appear that the "neglected" children identified in other studies would score low on "appropriate" social skill, while the "rejected" children would score high on "inappropriate assertiveness". The strategy adopted by the authors 
was to evaluate a wide range of verbal and non-verbal behaviours, emphasising interpersonal effectiveness without harm to others. Items of behaviour were drawn both from the child social skills literature and from the literature on children with behavioural difficulties.

\section{iii) Extraneous variables.}

Individual differences may effect outcome. Pentz (1981) found that in adolescents receiving assertiveness training, verbal reasoning and state anxiety accounted for more variance than training variables. Putallaz (1983) controlled for intelligence in assessing the outcome of her study on the effect of social skills training on sociometric status, thereby increasing the validity of the research. Hops (1983) notes that at times it may be necessary to add nonsocial skills to a child's repertoire, such as language competence or motor abilities. Handicap or disfigurement may be a cause of social difficulty, in terms of the "first impression" made on others by the individual. This area requires more attention from researchers.

It is necessary, then, when assessing outcome, to allow for extraneous variables which may lie at the root of the problem. This issue requires attention both at the individual level and at the level of the group receiving social skills training. 


\section{Planning for Generalisation}

Van Hasselt et al. (1979) express concern about the paucity of data concerning generalisation and maintenance of treatment effects. of the twenty single case designs which they review, ten report no follow-up. of the nine group designs, two report no follow-up. of all 29 studies, only 3 report follow-up beyond one month. Spence and Marzillier (1981) reported that they were able to produce some changes in targeted behaviours of young male offenders using a multiple baseline design, but these short-term changes in social skill did not lead to long-term changes in social competence as assessed by staff and other observers.

One possible approach to the problem of generalisation is described in the adult literature as "over-learning" (Shepherd, 1983; Goldstein et al., 1979). Along these lines, Rinn and Markle (1979) advocate that verbal instructions are emphasised in training children in social skills, and that various measures are taken to support the programme in the natural environment, including the involvement of parents and siblings in reinforcement procedures and taking active steps to ensure that clients have adequate opportunities for peer interaction. Verduyn et al. (1990) include "booster sessions" in their treatment package with pre-adolescent children. Hops (1983) proposes training clients in extraneous skills, e.g. extending their linguistic capacity, if such deficits seem to be impeding progress in social skills training. 


\section{Assessment of Generalisation}

Generalisation over time (maintenance) is typically assessed by follow-up, i.e. repeating the measures used to assess outcome. However, this may not be appropriate with young clients, as their rapid social development may require the emergence of new skills and behaviours rather than the maintenance of old ones. Trower's model of generative skill (Trower, 1982, 1984) may be more appropriate than a strictly behavioural model in assessing long-term gains. Social-cognitive and sociometric measures (e.g. Oden and Asher, 1977) may give better assessments of generalisation than purely behavioural measures. Verduyn et al. (1990) found evidence of generalisation by using a parent questionnaire, and it may well be worth developing this type of instrument to obtain more detailed information. Both sociometric and parent questionnaires have the advantage that they assess generalisation across situations as well as over time.

Assessment of generalisation can sometimes have surprising results. Dubow et al. (1987) carried out a comparative outcome study with 104 aggressive boys aged 8-13 from "behaviour disorder" classrooms. Clients were assigned to one of four treatment conditions: cognitive (self-control) training, behavioural (prosocial skills) training, combined cognitive-behavioural training, and attention/play "training". Only the cognitive-behavioural and attention/play conditions produced reduction in aggression and increase in prosocial behaviour post-intervention; and only the attention/play condition led to to maintenance of these improvements at 6 -month follow-up. The "anti-didactic" perspective of Yardley (1979) may help to explain this unexpected finding; we may suppose that rapport was rather better for this particular group of clients in the attention/play condition, and consequently a role-modelling process may have occurred unintentionally. 
Alternatively, the vital difference between conditions may have been one of motivation, as during the attention/play condition boys were allowed to play board and card games with each other in pairs. The researchers state that no cognitive or behavioural components were included but they do admit that some of the components of social skills training - instructions, coaching, roleplay, modelling, feedback and discussion - were used in these sessions. It is likely that these highly competitive youngsters found these sessions provided a particularly welcome outlet to their aggression, and therefore the situation was in fact intensively reinforcing of the nonspecific prosocial skill input provided in the sessions. This study raises the issue of possible effects of attention/play conditions in social skills training and also alerts us to the importance of possible interactions between client variables and treatment variables.

\section{Therapist Effects}

The advantages of offering social skills training in a school setting have been discussed in section 6 (Intervention/Setting). To facilitate such school programmes it may be helpful to involve teachers in working alongside experienced practitioners in implementing social skills programmes. Frosh (1983) has suggested how this may be done. There is evidence that "paraprofessionals" may be as effective as trained therapists (Berman and Norton, 1985). It is important however for therapists acquiring new techniques to be adequately supported by experienced professionals. 
Given the wide potential range of therapists, -it is important to analyse the effect of therapists on outcome. Wilkins (1986) argues that therapist traits, states and behaviours should be studied in their own right. They do not accept that use of a placebo group is sufficient to control for therapist effects, since in their view it is not possible for a therapist to be blind as to treatment approach.

In the context of social skills training, the training of separate behaviours may be evidenced in a multiple baseline design, but as indicated above (Section 6, Intervention/Components) there is little evidence from comparative outcome studies that one component (e.g. cognitive) works better than another (e.g. behavioural). Consequently, the possibility remains that reported improvements may be attributed to therapist effects.

There has been a good deal of research on therapist variables in adult psychotherapy and this has been reviewed in detail by Parloff et al. (1978), who conclude that non-specific effects in therapy (Jung, 1934; Rogers, 1957; Truax and Mitchell, 1971; Truax anà Carkhuff, 1967; Frank, 1973) are not clearly established but require further investigation. Whatever these effects may be, there is no doubt that they are also applicable to work with children.

In the adult social skills literature, Yardley (1979) asserts that one secondary effect of social skills intervention may be the psychological support given by the therapist to the client. In children, low self-esteem has been identified as a possible antecedent of social difficulty (Herbert, 1986; Coopersmith, 1967). The ability of the therapist to provide 
psychological support, thus enhancing self-esteem, may then be an important variable.

In the present research, attempts are made both to control and to monitor therapist effects.

\section{The Individualised Approach}

The term "individualised approach" is here used to denote a procedure in which a social skills programme is tailored to the specific training needs of the individual. In a "standardised approach" one or more individuals would be identified as requiring help, and would be given a "standard" procedure, without any further attempt to take the individual's particular needs into account.

Single case designs (eg the multiple baseline design) have been found useful for documenting change during the process of training (e.g. Bornstein et al., 1977). However group training is commonly used with older children and adolescents in order to take advantage of peer modelling. The danger is that within the group context, the focus on individual training needs may be lost.

Marzillier and Winter (1978) have looked in detail at the individual differences between adult clients which appear to be crucial in determining outcome. They found that in some cases it was necessary to "desensitise" anxious patients prior to the commencement of social skills training. Pentz (1971) found that individual differences in adolescents made an important difference to the variance. All authorities acknowledge the importance of systematic assessment for social skills training, and this would seem to 
imply that allowing for individual differences (e.g. in personality, intelligence, and anxiety level) is an integral part of the intervention.

Hymel and Asher (1977) investigated the effects of training unpopular preadolescent children with one of three procedures - first, the previously described coaching procedure reported by Oden and Asher (1977); second, a more individualised coaching procedure, which emphasised specific concepts that particular children seemed to need; third, a procedure which paired children for play but provided no instruction. Children in all three groups made sociometric gains at post-test and six-month follow-up. In the absence of a control group it was not possible to determine whether or not the gains were to be accounted for by a regression towards the mean.

There was no significant difference in the gains made by any of the three groups. Hymel and Asher suggest that their failure to reproduce the findings of Oden and Asher (1977) may have been due to a difference in age, as some older children were included in their study. In the case of the study by Oden and Asher (1977), the coaching group made significantly greater sociometric gains than did the peer pairing group.

Hops (1983) advises using individual reinforcement contingencies within a group context. For example, peers may reciprocally provide social reinforcement to each other for attaining their agreed targets. This approach helps with generalisation in the natural environment. The present investigation explores a related process empirically in Experiment 2, where the independent variable in the design is the degree to which target setting in the natural enviromnment ("Homework setting") is individualised within the context of a group training design; the dependent variable is 
outcome. A more complete explanation will be. offered at the appropriate point.

\section{Conclusions and Implications}

i) Origins

Social skills training is derived from a number of sources, notably the social learning model (Bandura, 1977), the Argyle model (Argyle, 1967), the assertion training model (Wolpe, 1969), and analysis of "micro-skills" (Trower, 1979). The mix of theoretical ingredients is potent, heady, and perhaps uncertain in its effects. In the first ten years of its development, the technique generated a good deal of excitement, as new movements do; now it is perhaps time to settle down to some sober evaluation.

\section{ii) Definition}

Owing to the empirical and behavioural nature of the approach, it is difficult to define social skills training other than by the techniques which it claims to subsume. Typically, these include verbal instructions, modelling, roleplay, feedback, and homework assignments. The approach is based on the notion that individuals may suffer from deficits which may impair their social functioning in some way, and therefore training in the requisite skills will help them to overcome these deficits and lead a more satisfactory life. The "axiom" which underlies the approach is the belief that individuals can be trained to improve their skills in social interaction. There is research evidence that early problems in social interaction are associated with various difficulties in later life, but 
opinions vary as to how this should be interpreted.

Our knowledge of the specific skills required by specific individuals to cope with specific situations is still very limited. Practitioners must therefore undertake field studies to explore these areas; otherwise the skills they teach may be irrelevant.

\section{iii) Components}

We have little understanding of the various cognitive and motivational processes which mediate the acquisition of skill in the field of social interaction. Intuitively, it would seem appropriate to mobilise human cognitive potential in the service of successful social interaction. Research has taken an interest in this matter but has not yet provided a clear directive. There has been some useful exploration of the concept of modelling. Problem-solving ability, knowledge of social strategies, selfesteem and anxiety are clearly relevant and from a theoretical point of view Bandura's concept of self-efficacy and White's notion of a developing sense of competence/mastery may be useful in integrating some of these constructs.

There are sound arguments for following McFall (1982) in making a distinction between social competence and social skill. Social competence may be defined as observed performance on a given task or range of tasks carried out within a "real life" context; social skills are conceptualised as the abilities required by the individual in order to attain social competence at the criterion level. Judgements as to social competence are therefore to be regarded in the strict sense as "criterion referenced"; however, social norms might be taken into account in setting the criteria. For example the competence criterion "Able to socialise with peers" might 
subsume the ability to respond with developmentally appropriate behaviour when in the presence of a peer (i.e, a specific social skill).

In practical terms, trainers or therapists need to be clear about what help they are offering. This could vary from helping a client to carry out a discrete task more effectively, to facilitating a major development in personality. Presumably Kelly's fixed role therapy is a form of social skills training (Kelly, 1955); it certainly involves training a client in social interaction.

\section{iv) Applications with children, adolescents and young people}

Social skills training, like assertion training, may be helpful to college students who are shy and lonely. The same, one would think, must be true in the case of younger adolescents from different social backgrounds who suffer from this type of difficulty, though the research evidence for this is sparse. Similarly, there are grounds for expecting that social skills training might help young adolescents who are perceived as antisocial or unhelpfully aggressive to develop a more effective, "prosocial" type of response. Again, evidence is limited. Social skills training does not seem to "cure" delinquency, but may have a place in the rehabilitation of delinquents. 
From the three studies which have been reported in some detail, it would appear that preadolescent youngsters may benefit from social skills training, both in terms of immediate behaviour change and more long term gains in competence. The number of studies is limited and replication of this work is required. With this age group, both individual and group training methods have been used. With younger children, most studies have used individual training methods and the emphasis has been on shaping, modelling and peer-mediated strategies.

\section{v) Antecedents}

The antecedents of social skill difficulty may include faulty reinforcement contingencies, low self-esteem, inconsistent feedback in infancy, and an insufficient sense of mastery of the environment.

\section{vi) Type of problem}

It is useful to divide socially unskilled children and young people into two categories: those who are aggressive and disruptive or "overassertive" and those who are variously described as isolated, unassertive and withdrawn. Difficulties may be mild or severe in either category. The first type are commonly called "rejected" and the second "neglected". This is because the the first type receive negative nominations on sociometric assessment procedures, while the second typically fail to receive positive nominations. It is thought that the rejected group have a poorer prognosis, and this is likely to be true, since both groups are known to be inept in social interaction, but the rejected group have a habit of responding antisocially which must compound their problem. 
In assessment for social skills training a mix of measures should be used sampling the various levels of social functioning a) behaviour/skill/performance, b) social cognition, c) social competence. In children and young people social competence may be most reliably judged by observers who are familiar with the individual in the natural environment.

\section{viii) Intervention}

Interventions must take account of the developmental level of the individual. For older children and young people, the balance of the evidence suggests that a comprehensive treatment package delivered in school offers a reasonable chance of success. A group training procedure enables the peer group to be used for reinforcement, but it is important that the needs of the individual should not get lost. Therefore the intervention must be very carefully planned. Planning should aim for generalisation.

\section{ix) Assessment of outcome}

In assessing outcome, both single case and group comparison designs are useful. It is important to control and monitor therapist effects, and the conventional method of using an attention placebo group is open to the criticism that therapists cannot be "naive." Generalisation as well as short-term gain should be assessed. 


\section{Towards Implementation}

The present research addresses the issues raised by the literature in the following ways:

i) Adolescents - an underresearched group - are selected for special study. With the exception of delinquents, this client group has received little attention. There are very few studies of young adolescents, the population studied in the present research.

The literature suggests that socially diffident college and university students may require and benefit from social skills training. There is a considerable body of evidence for the effectiveness of social skills training with children. It is important to establish whether adolescents also may benefit.

Adolescence is a major developmental stage in the life cycle. It is often a time of stress, particularly in our society where young people continue in education and training after puberty, and are therefore frequently in a dependent role in spite of their physical maturity. There is evidence to suggest that improvement in social functioning early in life may prevent later psychological disorder. Therefore intervention at adolescence may be of considerable importance.

ii) The present research provides social skills training within a school setting. This approach offers a number of advantages, two of which may be briefly mentioned here. Firstly, offering training within a natural setting facilitates the process of generalisation. Secondly, many of the difficulties encountered at this age by socially unskilled individuals 
occur at school - e.g. teasing and bullying.

iii) The present research uses a comprehensive treatment package. Whilst there is no "hard" evidence, the consensus of the literature would be that this is the most appropriate approach for young adolescents. There is a need to develop suitable packages for this client group.

iv) The present research provides a group training approach. This is cost-effective in terms of professional time, and enables peer-modelling to occur under the skilled guidance of the trainer.

v) The present research tests the hypothesis that an individualised approach may enhance the effectiveness of the group training procedure. In the individualised approach, there is no additional contact between trainer and client; the didactic content is the same, but the "homework" assignments and subsequent feedback are more geared to individual needs.

vi) The present research seeks to provide social skills training to both underassertive or "neglected" and overassertive or "rejected" youngsters concurrently within the same group training procedure.

vii) The present research controls for the possibility that outcome may be related to therapist variables. 
SOCIAL SKILLS TRAINING WITH YOUNG ADOLESCENTS

Chapter Two

Study I

Table of Contents

Section

Title

1.

Introduction

2.

Method

3.

Results

4.

Discussion

5.

Conclusion 
SOCIAL SKILLS TRAINING WITH YOUNG ADOLESCENTS

Chapter Two

Study I

\section{Introduction}

\section{Aims of the research programme.}

The research programme had the following 5 broad aims, based on considerations which have been dealt with fully in the literature review.

i) To test the hypothesis that social skills training may be effective with young adolescents. This is an under-researched area (see "Areas of application" in the literature review).

ii). To assess the feasibility of providing such training within a school setting. Some advantages of this approach have already been mentioned (see "Intervention"); others are given below (see "Rationale").

iii). To evaluate the assumption that an appropriate means of delivering such training would be through a comprehensive group training package (see "Intervention" with special reference to "Group vs. individual training").

iv). To explore the possibility that within that package, an 
"individualised approach" might produce the optimum results (see "The individualised approach").

v), To evaluate the need for controlling "therapist effects" in designing and implementing such programmes.

Study I is concerned with aims (i), (ii), and (iii); study II seeks to check the conclusions of Study I and to address aims (iv) and (v). With respect to aim (iv), individualised training is included in the treatment package in Study I; study II makes an attempt to isolate the contribution made by individualised treatment, by assigning some subjects to an "Individualised Training" condition, and others to a "Standardised Training " condition.

\section{Description of Study I}

As indicated in the literature review, there is some evidence that a relatively comprehensive social skills training package may be appropriate in a school environment. In Study $I$, such a package was run with 9 socially unskilled youngsters aged 12-13, and their results were compared with those of a non-equivalent control group (i.e a comparable group of youngsters without identified social skills difficulty).

Partly by way of recapitulation, and partly to develop the point further, the grounds for selecting this mode of intervention are here considered in some detail. 


\section{Rationale.}

The choice of early adolescence for an intervention is based in part on developmental considerations. Children at this point in their lives are entering on a new maturational stage. They have (in many cases) recently entered a new, much larger school, which may present them with difficulties of social adjustment. They are beginning the process of adolescence, in which the salience of the peer group tends to increase at the expense of adult role models. Rutter (1985), looking at more general problems of childhood adjustment, emphasised the importance of preventative intervention at certain key stages of children's development. Such developmental considerations support the view that early adolescence may an appropriate time for the application of social skills training techniques.

A number of studies have been mentioned in the literature review as examples of the use of group training methods with this age group. In clinical work the approach has been found to be effective. Young people are often ready to respond to the influence of the peer group, while they may view the adult world with suspicion or resentment. The potential of the peer group for supporting change has been demonstrated extensively in the social skills literature on children. Further, group training provides an ideal opportunity for role-play - one of the central techniques in social skills training - and for "ice-breaking" games, which allow young people to. experience the breakdown of social barriers and the release of tension within a controlled situation. To treat social difficulty, it makes sense to employ controlled exposure to a reasonably supportive social situation in remedial training. 
Exploratory studies have suggested that school may be a suitable setting for social skills training (Bowdler and Gleisner, 1982; Pellegrini and Urbain, 1985; Verduyn et al., 1990). The potential for change in terms of immediate reinforcement in the natural environment is an advantage (Hops, 1983). The removal of the child to a clinic is to be avoided if possible as it may cause stigmatisation, thereby compounding the problem.

It may be argued that training in school would also cause stigmatisation. However this may be presented as a form of educational specialisation, while attendance at a "clinic" frequently carries for young prople the undesirable connotation of being identified as a "mental case" and therefore entails a risk (real or perceived) of increasing social difficulty.

Finally the administrative resources of a school are helpful in setting up selection procedures and ensuring the proper implementation of assessment measures. For example, the school organisation may be used to carry out sociometric investigation of a whole year group, i.e more than 100 students. This would normally be a time-consuming and therefore expensive research procedure.

To summarise, there are sound reasons in clinical, educational and developmental terms for carrying out systematic social skills interventions within school, using the method of group training, and the approach is supported by published research. 


\section{Measures.}

The outcome measures used in Study one were sociometric nomination, staff assessments of social competence, and self-report questionnaires.

It has been explained that there is a difficulty in making detailed behavioural observations with this age group. (See "Assessment" in the literature review). Further, adolescents may not be reliable in keeping social diaries. Jackson and Marzillier (1983) in a study of 16 adolescents reported that "Only seven subjects and seven parents returned the completed diary at post-test, rendering statistical analysis of doubtful validity" (p. 312). Role-play techniques may demonstrate change across sessions (Bornstein et al., 1977) but these authors acknowledge that generalisation to "real life" may not be assumed from such data. The literature review has indicated that the status of role-play measures in the assessment of social skills is controversial (see "Assessment"). Hops (1983) propounds the view that multiple subjective measures of "social competence" le.g. parent and teacher questionnaires, peer sociometric ratings) may be an extremely valid approach to assessment of outcome in social skills training. Children referred to mental health centres can be discriminated from non-referred children on the evidence of their parents' ratings, and peer group social judgements have been validated by adult social judgement later in life (Hops, 1983; also see "Developmental approach/ Rationale" in the literature review).

The current study therefore (Study I) depends principally on subjective peer and staff observation coded into judgement on questionnaires rather than on direct measurement of behaviour to complement the data available from self-report questionnaires. Undoubtedly behavioural information would 
have been of some value; however both the theoretical considerations outlined above and the practical constraints of limited resources led to the exclusion of behavioural measures on this occasion. In clarification it should perhaps be stated that behavioural observation (e.g therapist observation of role-play or "behavioural rehearsal") was used in this study as a training technique within the intervention but not as a measure of outcome.

\section{The control group.}

From the viewpoint of pragmatism, it was felt appropriate that a "nonequivalent control group" (Kenny, 1975) should be used to check that changes noted in the treatment group would not have occurred naturally in a "normal" group of this age. Kenny advised that a non-equivalent control group should be used in natural settings where randomisation was not possible. In the present study, it would not have been possible for some clients requiring help to be identified, and then randomly allocated to two groups, one of which would receive help, and the cther would not. Arguably this would have been better research; but it would not have been acceptable at this time to educational colleagues, on whose help this experiment depended. Partially on pragmatic grounds, therefore, it was decided that a "non-equivalent control group" would be better than no control group at all; there is certainly a case for such a group in spite of it $s$ limitations. 


\section{Method}

The project was conducted in a Community School providing for the education of some 600 pupils of both sexes. The school was in a New Town area serving a socially mixed population. The subjects fell within the 1213 age range. There were two groups. The treatment group ( 6 boys, 3 girls) was selected on the basis that each member had an identified social skills difficulty (eg "isolated, unable to make friends") as established by staff nomination and by obtaining a criterion score on the social competence questionnaire (see below); the control group, whilst being of like age to the treatment group and similarly balanced for gender ( 5 boys, 5 girls) had no identified social skills difficulty. All assesssment measures were administered to both groups.

\section{Range of client difficulty.}

Clients' social difficulties ranged from moderate to severe. Clients had difficulties both with adults and with peers. The sample included both "rejected" and "neglected" clients, though the use of negative peer nominations for selection was excluded on ethical grounds. Details of clients' social difficulties are briefly given below.

client 1 (12 years 8 months). Male; severe difficulty with adults, moderate difficulty with peers (Teacher questionnaire). No evidence of difficulty from self-report or sociometric questionnaire.

client 2 (13 years, 0 months). Male; high academic ability; severe difficulty with adults, moderate difficulty with peers (Teacher questionnaire). Moderate difficulty shown on self-report questionnaire. No 
lack of sociometric nominations, but self-report data suggest he is "rejected".

client 3. (13 years, 1 month). Male; severe difficulty with both adults and peers (Teacher questionnaire). Low self-esteem. Moderate difficulty on self-report questionnaire; appears "rejected", though no lack of sociometric nominations.

Client 4. (12 years, 7 months). Male; severe difficulty with both adults and peers (Teacher questionnaire). Low sociometric status, so "neglected". No difficulty shown on self-report questionnaire.

client 5. (12 years, 3 months). Male; high academic ability; moderate difficulty with peers, severe dificulty with adults (Teacher questionnaire). Described as "Disorganised". Substantial difficulties on self-report questionnaire; evidence of both "rejection" and "neglect" present. Low sociometric status. Referred for psychological difficulties.

client 6. (Age comparable, but not recorded). Male; low academic ability. Severe difficulty with both peers and adults. Substantial difficulty on self-report questionnaire; evidence of both "rejection" and "neglect". Low sociometric status.

Client 7. (12 years, 8 months). Female; mild difficulty with peers and adults (Teacher questionnaire). Moderate difficulty on self-report questionnaire. Low sociometric status. 
Client 8. (12 years, 6 months). Female; moderate social difficulty with peers and adults. Described as an "isolate". Substantial difficulty indicated on self-report questionnaire; evidence of both "rejection" and "neglect".

Client 9. (12 years, 6 months). Female; moderate social difficulty with peers and adults (Teacher questionnaire). Moderate difficulty on selfreport questionnaire. Low sociometric status.

\section{The therapists}

Group sessions were run jointly by a psychologist and two teachers - one teacher was a member of the school staff, and the other normally worked in a clinical setting. Two of the therapists were experienced at running social skills programmes with this age group and the third was an experienced teacher. Two of the therapists were male and one was female. Planning meetings were held prior to each session and review meetings immediately afterwards.

The two experienced therapists had run a number of groups together with this age group both at a clinic and in school. They had experience of training youngsters with a wide range of social skills difficulty including both the "rejected" and the "neglected". They had established roles which were to some degree complementary, one being "task oriented" and the other "person oriented" but were able to interchange their roles. The third therapist was not experienced in the technique but was enthusiastic to 
learn about it and prepared to commit time and energy to the project. As a regular member of school staff, the third therapist had the role of liaising between the project and the school.

\section{The assessment measures.}

The assessment measures included (a) a social competence questionnaire, completed by the teachers; (b) a self-report questionnaire, in the form of a list of social situation problems; and (c) a sociometric procedure, administered to the whole age group of which the treatment and control groups each formed a part.

\section{(a) The social competence questionnaire.}

The social competence questionnaire was designed specifically for this project (See Appendix 5). It was completed by a member of staff who was well acquainted with the student to whom the questionnaire referred.

The first part of the form reqested a rating from $A$ to $E$ on Intellectual Potential, Academic Performance, and 13 items of social skill, e.g "Ability to mix with peers", "Asking a question", "Keeping a conversation going", "Following instructions", "Asking for help".

The second part sought to elicit details of the occurrence of social skills difficulty, e.g. "Where does the problem behaviour happen - in school or outside?" "If in school, in what situation?" "How often does it occur (eg how many times per week)". 
Other items sampled the frequency of peer group difficulty and asked for information about self-control.

The form therefore provided a quantitative assessment of social skill difficulty together with useful clinical information of relevance to the presenting problem.

(b) The self-report questionnaire.

The self-report questionnaire used was the " List of social situation problems" designed by spence (1980) for use in institutions and in the community. This questionnaire is of the "Yes/No" type and contains a list of sixty situations which may or may not be experienced as difficult. It includes items involving relationships with both peers and adults and covers areas such as social anxiety and difficulty with authority, eg " Do you feel shy with strangers", "Do you find it hard to say you are sorry to members of staff". The validity of the questionnaire rests on the assumption that students in this age group are co some degree able to identify the problems that they experience in social interaction.

\section{(c) The sociometric procedure.}

In the sociometric procedure students were asked to nominate a member of their own class group whom they would choose as a partner or a companion in each of four activities (Macmillan et al., 1980). These included "work" activities and informal "leisure" activicres. Only positive nominations were requested. 


\section{Intervention.}

Within the training programme, behavioural methods were employed intensively. Individual needs were analysed in some detail on the basis of the self-report questionnaire (Spence, 1980) and on role-play performance. Individuals were divided into sub-groups for some role-play sessions (e.g. "Coping with being teased", "Negotiating", "Being left out", "Asking permission"; see Session Formats, p.17, for full details) in order to rehearse socially appropriate, assertive behaviours. Part of the group training was allocated to individual "goal setting" sessions in which individuals were advised of the behaviours which they most needed to practise (see Session Formats, p.17). Thus, within the group training programme, care was taken to cater for the needs of individuals.

The group training was given in a comfortable room with ample space for role-play and video equipment on hand. Sessions lasted one hour and a quarter and were held weekly (see Course Plan, p.16, for full details). The total treatment package consisted of ten sessions. Sessions generally began and ended with games and "ice-breakers". In the initial sessions, work was done on verbal and non-verbal "micro-skills"; (for example, the sequence "Greet a friend across the street" was role-played and the non-verbal and verbal elements of the interaction were analysed with the group.) In later sessions there was a shift to working on the type of situation which might cause stress to clients (e.g. dealing with bullying or malicious teasing, standing up for oneself, joining a new group, dealing with authority). These situations and the skills necessary for dealing with them were roleplayed and then analysed in some detail. In this way, clients were given an opportunity to learn new skills through practice, participation, observation and discussion. (The use of simulated situations has been 
found useful in other areas of learning, both practical and social - for example in the use of a simulated sailboard in learning to windsurf, and in management games.)

Clearly, an advantage of this type of learning situation is that immediate feedback on performance in the simulated situation can be given both by the trainer and by peers, thus enabling the client to modify and improve performance within the context of a relatively "safe" situation. Positive reinforcement was used whenever possible by trainers to strengthen appropriate verbal and non-verbal responses on the part of clients. Reinforcement by peers (a particularly powerful motivator) was also strongly encouraged. "Homework" assignments were set for clients, in order to stimulate them to practise new styles of behaviour in real life. Appropriate real-life tasks were discussed with each client, and clients were subsequently asked for feedback on the tasks that had been set. It was hoped that experience of success in such tasks would provide much-needed reinforcement for new, more appropriate styles of behaviour. In addition, it was hoped that the experience of reporting and receiving positive feedback would begin to train clients in the process of self-reinforcement for the desired behaviours. Full attention was given to individual needs in each of the above activities. Programming for generalisation was included in the final sessions. 
The details of the intervention are summarised in the Course Plan, p. 16 , and Session Formats, p. 17. In the Course Plan, it can be seen that roleplay assessment forms an important part of the early sessions. By this stage, the therapists have some idea of where the clients are failing in terms of their social competence; the role-play may be used to obtain detailed information about the social skills deficit underlying the failures.

By the mid-point of the course, individual assessments are complete. It is then possible to plan training, based on the individual assessments. Task rehearsal is used to prepare clients for carrying out the "real life" homework assignments. The aim of the therapy is to produce change in a specified direction in real life such that skill in an identified deficit area is increased. It is important both to set homework and obtain feedback on the homework; in this way, change is produced and evidence of the change is acquired. At the same time reinforment can be provided and processes of self-reinforcement may be initiated. By prior task rehearsal the therapists ensure that the response is present within the client's repertoire. The therapeutic activity creates a mental set for clients to rehearse skills cognitively in the future and thereby enhance their ability in social problem-solving.

At the end of the course progress is reviewed and feedback is given on the final homework assignments. 


\section{Course Plan}

Overview

Session No.

Function

Method

1.

Induction

Assessment

Assessment

Observation

Assessment

Training

(based on

assessment of

individuals)

7/8/9

10.

Individual

homework

Conclusion

Re-evaluation
Contract

Questionnaire

Discussion

Roleplay

Roleplay

Role-play

Coaching

Feedback

Reinforcement

Task rehearsal

Feedback on task

Reinforcement

Review progress

Role-play

Questionnaires 


\title{
Structure of Sessions
}

Each session is planned in detail, having regard to the progress made by individuals, specific needs for assessment and training, and the stage reached by the group as a whole. The aim in each session is to provide a pleasurable group experience and to enable the group members to assist each other by (a) participating in role play (b) offering feedback and reinforcement.

The list of Session Formats provides an indication of the activities which make up the course.

\section{Session Formats}

1.

\author{
Introduction \\ Name Game \\ Blind Partners \\ True Story \\ Contract
}

2.

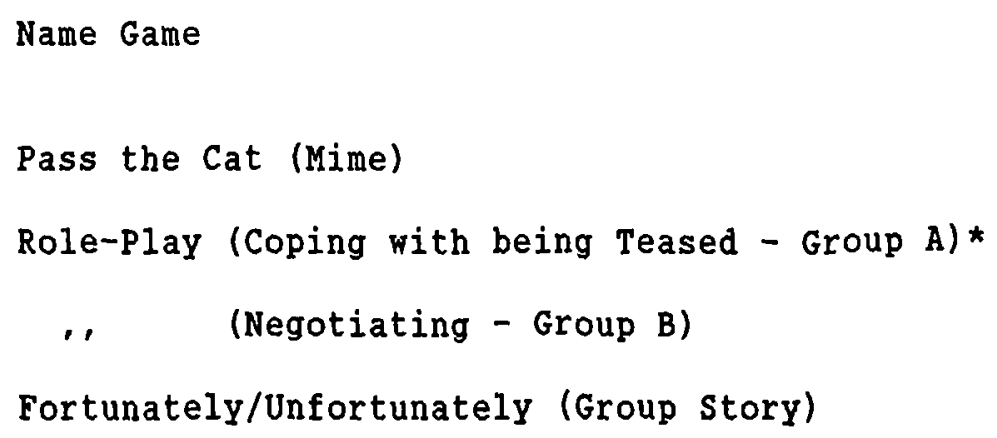


3.

4.

5.

6.

Introduction

Goal setting

Looking Meditation

Saying No

Role- Play (Dealing with a Difficult Situation)

Feedback on Behaviour during Session 
7.

Expectations (for Individuals in Session)

Meditation

Individual Task setting

Modelling

Role-Play Practice

Feedback

Assignments Given on Card

Feedback on Behaviour in Session

Fortunately/ Unfortunately

8.

Expectations (Reminder of Outing)

Meditation

Feedback on Assignments

Reinforcement (Stars on chart and verbal praise)

Task Setting

Role Play (Task Rehearsal)

Feedback on Behaviour in Session

9.

Feedback on Final Assignments

Reinforcement

10.

Review of Individual Work

Reinforcement

Goal Setting for Future

* Groups were split here and later according to individual need. 


\section{Results}

\section{Sociometric nomination.}

As expected the treatment group $(\mathrm{n}=9)$ initially received significantly fewer positive nominations $(U=4, p) .05$, Mann-Whitney) than the control group $(\mathrm{n}=10)$. From the pre-treatment measure to the post treatment measure, the treatment group made no significant gain, but its gain was significant from pretreatment to six-month follow-up $(T=8, p>.05$, Wilcoxon). The control group made no significant comparable gain from pretreatment to follow-up. There was no significant difference between the gain scores of the treatment group from pretreatment to follow-up and the gain scores of the control group over the same period. However the treatment group had more gains and fewer losses than the control group over this period. One member of the control group in particular was identified as an isolate and made no gains. Consequently there was limited evidence of the usefulness of the intervention from this measure.

Breakdown of means and standard deviations.

The means and standard deviations of both groups on each of the other two measures are given in Table 1. 


\section{Table 1}

Means and Standard Deviations for Behavioural Measures for the Treatment Group and Control Group at Pretest, Posttest and Follow-up.

\begin{tabular}{lcc} 
Time of Assessment & Groups \\
and & Treatment & Control \\
\hline
\end{tabular}

Pretest

\begin{tabular}{|c|c|c|}
\hline Social competence & $13.78(3.53)$ & $8.00 \quad(8.49)$ \\
\hline Self-report & $23.33 \quad(9.29)$ & $21.87(7.85)$ \\
\hline \multicolumn{3}{|l|}{ Post-test } \\
\hline Social competence & $11.22(4.49)$ & $8.25 \quad(7.69)$ \\
\hline Self-report & $17.56(11.89)$ & $20.0 \quad(6.5)$ \\
\hline
\end{tabular}

Follow-up

\begin{tabular}{|c|c|c|}
\hline Social competence & $10.56(5.52)$ & $8.38 \quad(7.8)$ \\
\hline Self-report & $15.11(11.75)$ & $20.62(4.24)$ \\
\hline
\end{tabular}

Notes: (1) Standard deviations are in parenthesis.

(2) On both measures a reduction in score means an improvement. 
Using t-tests, the treatment group was compared with the control group on each measure for each occasion. A significant difference at pre-test was found for social competence $(t=1.79, d f=7, p<.05,1-t a i l e d)$. This finding indicated that the treatment group did in fact have more social skills difficulty than the control group, as judged by teachers. There was no significant difference found between the two groups at post-treatment and follow-up on this measure.

\section{Comparison of groups for degree of change}

Using multivariate analysis of variance (repeated measures) the pooled outcome measures (excluding sociometry) were analysed to see whether time had a significant effect and to see whether this effect was the same or different for the two groups. The effect for time was significant $(F=3.36$, $d f=4.0,12.0, p<.05)$. However, the effect for group $x$ time was not significant.

\section{Control group: Evidence for "no change".}

There was no significant change over time in the scores of the control group on any measure. 


\section{Treatment group results: Evidence for change.}

The evidence for improvement in social competence and self-report from pretest to post-test and from pretest to follow-up is given in Table 2 . Improvements in social competence were found to be significant at the .05 level from pretest to post-test and from post-test to follow-up. Improvements in self-report were significant at the .05 level from pre-test to post-test, and at the .01 level from pre-test to follow-up.

The evidence is presented visually in Graphs 1 and 2. 


\section{Experiment 1. \\ Graph 1 : Teacher Assessed Social Difficulty}

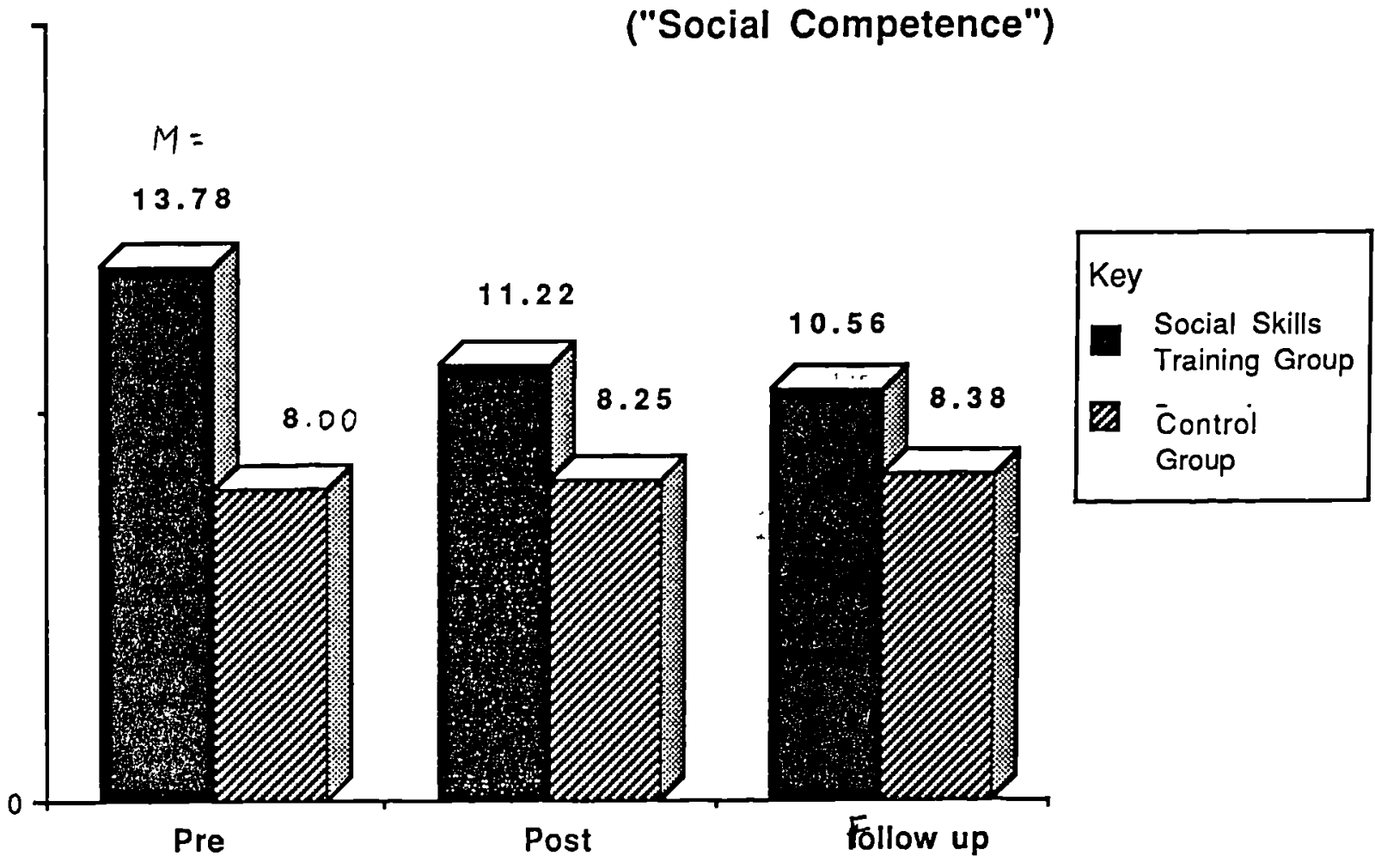

\section{Graph 2 : Self-Reported Social Difficulty}

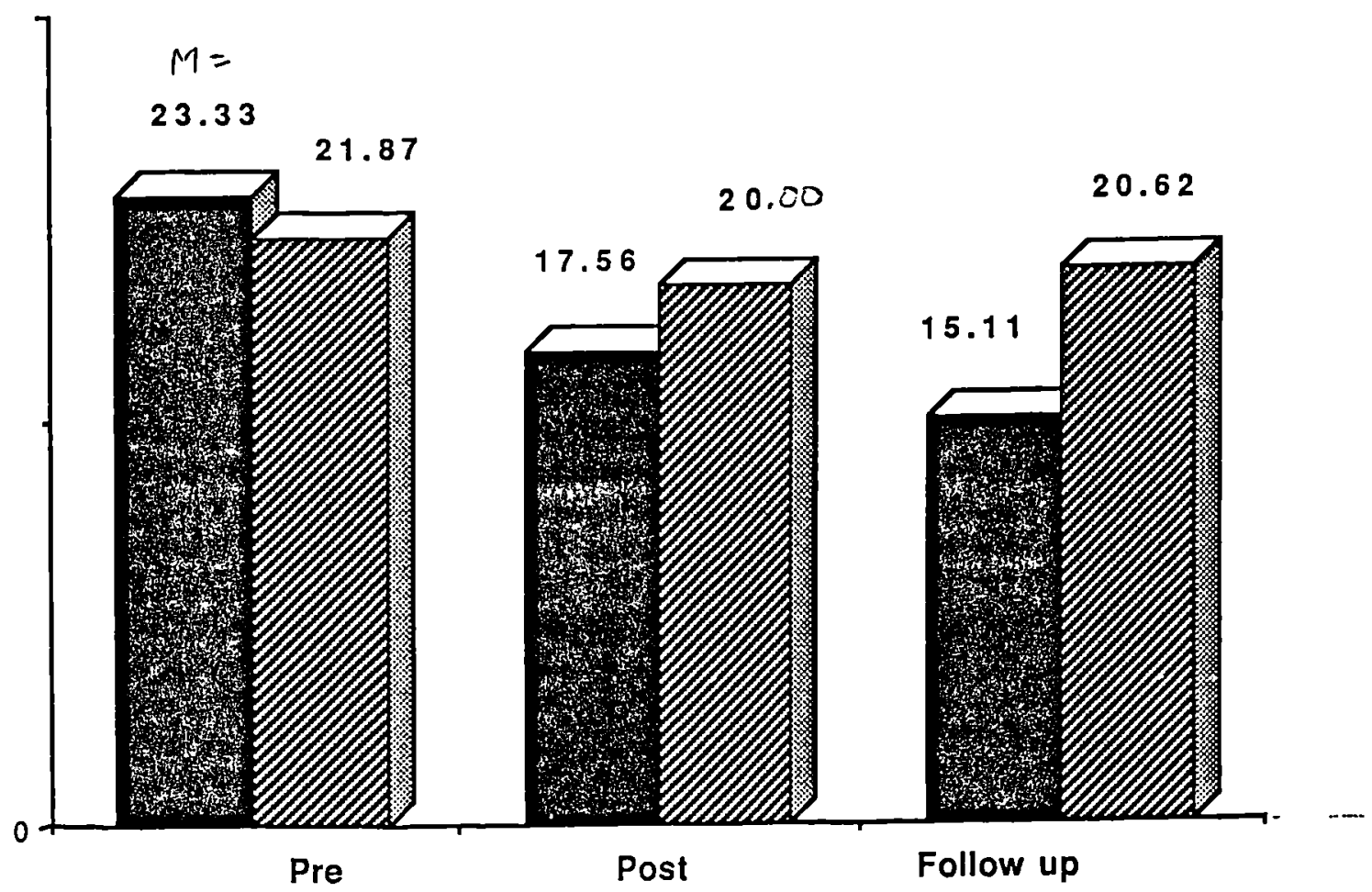


Table 2

Evidence for Improvement of the Treatment Group in Social Competence And Self-report

$t$

p (1-tailed)

Social competence

$\begin{array}{lll}\text { Pretest to post-test } & 2.55 & <.05 \\ \text { Pretest to follow-up } & 1.80 & <.05\end{array}$

Self-report

$\begin{array}{lll}\text { Pretest to post-test } & 2.56 & <.05 \\ \text { Pretest to follow-up } & 2.79 & <.01\end{array}$

Note: (1) $\mathrm{df}=8$ in each case.

(2) 1-tailed $p$ is appropriate, as the direction of change was predicted. 
Parent involvement.

The training procedure was reviewed with parents after completion. The general impression gained was that the attitude of parents to the project was positive. By conducting the project in school it was possible to have this contact with parents as an additional procedure to promote the generalisation of the social skills training.

\section{Discussion}

\section{Difference between groups: Composition.}

There were significant differences between the treatment group and the control group initially in terms of positive social nomination and teacher assessments of social competence. However there was no significant difference in the case of the self-report questionnaire, though the mean of the treatment group was of a greater magnitude than the mean of the control group indicating a higher awareness of social difficulties within the treatment group. It seems likely that there were in fact behavioural differences between the groups, giving rise to the differential judgements made by staff and peers. The non-significance of the self-report finding may then reflect a lack of awareness or defensive denial on the part of the treatment group in respect of their social ineptitude. Alternatively, the teacher and peer judgements might be a response to non-behavioural elements such as intellectual ability, physical attractiveness, or being new to the school. At all events, the control group was significantly better than the treatment group on two of the measures before treatment and not afterwards, supporting the hypothesis that treatment was beneficial. 


\section{Changes in self-report.}

The results suggest that the treatment group perceived themselves to be more socially skilled at the end of treatment than they did at the start of treatment, and that they retained that new perception at follow-up. Now self-report data are often criticised on the ground that the results could be influenced in some way by the subjects' motivation and perception. For example, they might try to please the investigators. However, there is no evidence that the treatment group perceived that they had changed. The measure used was not a rating scale but a sixty-item Yes-No questionnaire, used for research purposes. Consequently, it is not likely that this finding is to be accounted for by a desire to please the investigators, or by any other factor involving the motivation of the subjects.

It has already been stated that the validity of this questionnaire rests on the assumption that to some degree, subjects at this age are reliable informants on their own behaviour and emotional reactions involving social skills. At the start of treatment subjects would be likely to under-rate their difficulties, and at the end, as a result of raised awareness, they might make a more realistic self-assessment. 
Change in teacher rating.

Significant changes were shown on the Social Competence Questionnaire, which required the teachers to rate the subjects on a number of variables involving social skills. The ratings were made by a number of different teachers who were not directly involved in the experiment and had only a limited awareness of it's aims. It is likely that the teachers completed the rating scales objectively and if they happened to recall the previous rating, made a professional judgement as to whether change had occurred. Teachers are after all skilled observers of their own students. No doubt having previously completed the rating scale they would have a slightly different attitude to the task on subsequent occasions; but this criticism could be applied to any repeated measures design. It is likely, then, that these results also are evidence of real behavioural change.

\section{Change in sociometric status.}

The treatment group did not show immediate improvement after treatment, but by the time of six-month follow-up they showed a significant improvement which the control group did not show, and in fact at this time the two groups were quite comparable in terms of their sociometric status. Since the control group was non-equivalent, the apparent gain could be accounted for by a regression effect. The social skills training group did not have a direct effect on the children's peer relationships, as the social skills training group was composed of students who came from different primary class groups. Consequently, the peers encountered in the training group were different from the peers comprising the "natural social environment". 
There is evidence from the literature (Van Hasselt, 1979: Gresham, 1981), that roster-rating questionnaires (involving ranking peers on an alphabetical list) may be a more sensitive measure than straight nomination. In fact the two measures may sample different aspects of social perception; roster-rating may detect acceptability, while positive nomination may relate rather to friendship choice. Now an increase in acceptablility may register moro quickly than a change in friendship pattern on the assumption that friendship patter 1 s may tke some time to negotiate relations. Further, there was no negative nomination in this study to avoid encouraging stigmatisation. Both these considerations would suggest that the measure may have been under-sensitive.

The gradual pace of change in the sociometric pattern, such that significant changes are only detected at six-month follow-up, supports the above argument. It would appear that sociometric change lags behind behavioural change, as might be expected.

\section{Difference between groups: Outcome.}

Whilst the treatment group did show significant improvement on all three measures, the result of the Manova investigation does not provide definitive evidence for differencs in change between the treatment group and the control group. However, this may be due to dilution of the interaction effect by the stable control group. The possiblity exists that most of the variance was already explained by the main effect. The sum of the evidence is fairly strong: on two of the three measures, there were significant differences between the groups before treatment, but by the end of follow-up, there were none. On all three measures, there were significant improvements in the treatment group by the end of follow-up; 
the control group showed no such improvement. In the case of sociometry, possible lack of sensitivity in the measure has already been discussed. With regard to the other two measures, inspection of the means and standard deviations suggests that while there was little improvement in the means of the control group, (in fact the scores dropped rather than improved on the Social Competence Questionnaire) the standard deviations on both measures were larger for the control group than for the treatment group. This is of course to be expected as the treatment group was selected in terms of a particular variable, namely social skills difficulty. Their scores could be expected to cluster more closely than those of a group that was not selected in terms of that variable.

\section{Generalisation.}

There was good evidence of (a) maintenance of training gains, and (b) generalisation of behaviour from the training situation to the natural environment, in that by the time of follow-up a significant improvement on each of the baseline measures had been achieved and maintained. There was generalisation across situations as well as over time, in that change was in effect reported by teachers, peers and the clients themselves, suggesting that behaviour had been observed in a number of different contexts. There was progressive improvement from post treatment to followup in the case of self-report and sociometric status. This supports the notion that social competence is a generative skill, and that processes initiated during intervention may be continued and developed by the individual if appropriate reinforcement occurs in the natural environment. 
The control group did not improve, suggesting that the evidence of generalisation was associated with the intervention.

\section{Components.}

In many ways the components of the intervention were the traditional ingredients of a social skills treatment package. One aspect which was perhaps new was the combination of a group training programme with an individually based orientation throughout the course. The individualised approach has been advocated in the literature (Marzillier and Winter, 1978; Hops, 1983) and has been a useful component of successful interventions with this age group (Bornstein et al, 1977: Oden and Asher, 1977) but has rarely if ever been used in conjunction with a group training design with this age group. It has been argued in the introduction that a group approach is useful with this age group for developmental reasons. The specific function of the individualised approach within the context of group training is explored in more detail in Experiment II.

\section{Logistical issues}

Running the programme in school premises and school time had the following advantages. Firstly the process of obtaining measurements was greatly facilitated by the assistance of school staff who completed questionnaires and administered sociometric procedures throughout the year group. School staff also communicated with parents about the programme. Secondly there was no cost involved as the school took the view that the programme was of benefit to pupils. Thirdly, an outing to a skating rink was organised for students in school time as a "reward" for cooperating with the programme. 


\section{Conclusion}

There was evidence of significant change in the treatment group on all three measures, with no comparable change in the control group. There was good evidence of generalisation across time and across situations. The results strongly suggest that a systematic social skills intervention in school, using a standard group training package, may be helpful to young adolescents with social difficulties. In the training there was considerable emphasis on catering for individual needs as detected in the course of the initial assessment. It may be that this component of treatment contributed to the success of the intervention. 
SOCIAL SKILLS TRAINING WITH YOUNG ADOLESCENTS

Chapter Three

Study II

Table of Contents

Section

Title

1.

Introduction

2 .

Method

3.

Results (Section One)

4.

Results (Section Two)

5.

Discussion (Results, Section One)

6.

Discussion (Results, Section Two) 


\section{Chapter Three}

Study II

\section{Introduction}

Aim of experiment.

Study I demonstrated that there was evidence that a social skills training package using a group approach might be effective with young adolescents within a school context. study II aimed firstly to substantiate this finding, and secondly, to obtain more detailed evidence regarding a hypothesis suggested by observations arising from study $I$, namely that when this package is used with this client group, a key ingredient of the intervention may be the process of providing maximum individual attention, based on assessed need for remediation, within the constraints of the group programme. It was envisaged that that this hypothesis might be tested by running two parallel treatment groups, again using a group training approach, but providing a standardised form of training in one group, and an individualised form of training in the other group. It was felt that a group comparison design would be appropriate, but that in view of the nature of the hypothesis, single case designs should also be conducted, since these might yield useful qualitative and psychometric data to supplement the statistical information. 
other areas of enquiry included the effect of gender on outcome and the effect of type of problem on outcome (i.e "rejected " or antisocial vs. "neglected" or unassertive clients).

\section{Method}

Therapist effects.

It appeared to be important to run the groups on a parallel basis to avoid order effects. Clearly, if the same therapist pair ran different groups, it would be difficult for the therapist to be naive as to the design (Wilkins, 1986). If different therapist pairs were used, an attention placebo group would be of no use, since difference between outcomes could be produced by difference between therapists. In response to these considerations, it was decided that there would be two pairs of therapists and each pair would run one group in each condition. To control for time effects, the conditions were crossed over, as shown below.

Pair A

Time 1

Standardised

Condition

Time 2
Individualised

Condition
Pair B

Individualised

Condition

Standardised

Condition

The therapists.

Two therapist pairs took part in the project. Therapist Pair A worked with the Individualised Training Group in the first year and the Standardised Training Group in the second year; Therapist Pair B worked with the Standardised Training Group in the first year and the Individualised 
Training Group in the second year. In other words, the therapist pairs were "crossed over" in the design. The therapists met the following criteria. Each pair had good rapport and previous experience of working together. All were enthusiastic about Social skills Training and one of each pair had limited previous experience of Social skills Training. All were experienced in working with this age group. Therapist Pair A were experienced family clinicians. They were of mixed gender. Therapist Pair $B$ were both male. They were practising teachers. Both therapist pairs received regular supervision throuqhout the project from a psychologist experienced in running social skills training groups. Session content was monitored by audiotape recording. On a self-rating scale administered mid-programme (See Appendix 6, "Therapist Self Rating") , therapists rated themselves on items such as "Involvement in the group", "Proficiency in modelling social skills," "Grasp of concepts", and "Management of programme". Non-parametric statistics indicated that there was no significant difference between therapist pairs in terms of their rating of their own competence.

\section{Measures. (An overview)}

The roster-rating questionnaire (i.e rating peers from a class list) was introduced in place of the nomination technique to assess sociometric status. It was hoped that this would increase the sensitivity of this measure. New social-cognitive tests were introduced, including a roletaking test, to provide more information about process variables. It will be recalled that the role-taking test is a cognitive test which samples the ability of the subject to take in imagination the social role of another.

Subjects.

The subjects were young people at the same Secondary School that was used 
for the pilot study, aqed 12-13 years. Young people from two successive school years took part in the study.

Selection of sample.

Each of the two year groups was screened to identify those young people who appeared to experience siqnificant difficulty in social interaction.

Screening measures.

i) Teacher nomination.

Teachers were asked to nominate children in each of two categories; firstly "overassertive/creates tension/alienates others" and secondly "socially 
isolated/unresponsive". Examples were given of behaviour which would qualify youngsters for inclusion in each cateqory.

ii) Self report questionnaire (short form).

A shortened form of the List of Social Situation Problems (Spence, 1980) was administered.

iii) Sociometric questionnaire.

A variant of the "Roster and ratinq" method (van Hasselt, 1979) was devised. Youngsters were presented with an alphabetised list of all their classmates and asked to place in rank order the five with whom they would most like to share an activity. It is possible to identify rejected children by asking for negative nominations but this procedure was excluded on ethical grounds. It was anticipated that inclusion of a rank ordering component would increase the discriminative power of the technique.

\section{Criteria for selection.}

i) Nomination by one or more teachers.

ii) Cut-offs were established to identify extreme measures on the Self Report Questionnaire and the Sociometric Questionnaire. (Approximately 15\% of the population of each year group were selected by these means for the project.) 
Allocation to groups.

The subjects ( 41 over the two years) were allocated randomly to the Individualised Training (IT) Groups ( $\mathrm{n}=7$ in each year) the standardised Training (ST) Groups ( $n=6$ in first year, 7 in second year) and the No Treatment Control Groups ( $n=6$ in first year, 8 in second year). Within each group subjects were of mixed gender in roughly equal proportion.

Experimental dẹsign.

i) Intervention.

The treatment groups and the control group were compared on measures administered before and after the intervention. The Standardised Training group received a varied social skills package (See Course Manual, Appendix 1) containing essentially the same ingredients as Study 1 (See Appendix 4). The format was the same (Introduction to concept/ warm-up/ main work (roleplay)/ closure (game), and the number of sessions was the same (10). The Individualised Training group (see Appendix 1) received a similar type of group training; they were not trained separately, but in the latter part of the course, they had an increased emphasis within the group on homework tasks geared to their individual needs. Therefore, they had a greater involvement in roleplay aimed at preparation for those particular homework or "real life" assignments. In other words, within the context of group training, the programme was tailored to clients' individual needs in a way which did not occur in the group receiving standardised Training. The therapists for the Individualised Training group received the following instructions: 
"Special assiqnments" will be set to enable practice in "real life" situations to occur. These special assignments will be geared to the special needs of individuals. The therapists will be provided with social skills assessment profiles on each of the youngsters and will make conscious efforts to provide appropriate training to individuals in accordance with their special needs, so long as the constraints of working in a group context are taken into account.

ii) Assessment measures.

The plan for administration of assessment measures is set out in Table 1. Initially, it was planned that there would be a follow-up component in the design. However, this was to some extent frustrated by industrial action in the school. It was possible to obtain only minimum follow-up data and these are detailed in the results section. Additionally there was some limitation on measures which could be used with the no treatment control group. Self-report measures together with social-cognitive data were obtained from the two social skills training groups, whilst sociometric data were obtained on all subjects. As a major aim of the study was to check for difference in outcome between the two treatment groups, the loss of control group data was regrettable but not disastrous. The staff questionnaire was used with the control group in the second year. 
Experimental Design

(Assessment Measures)

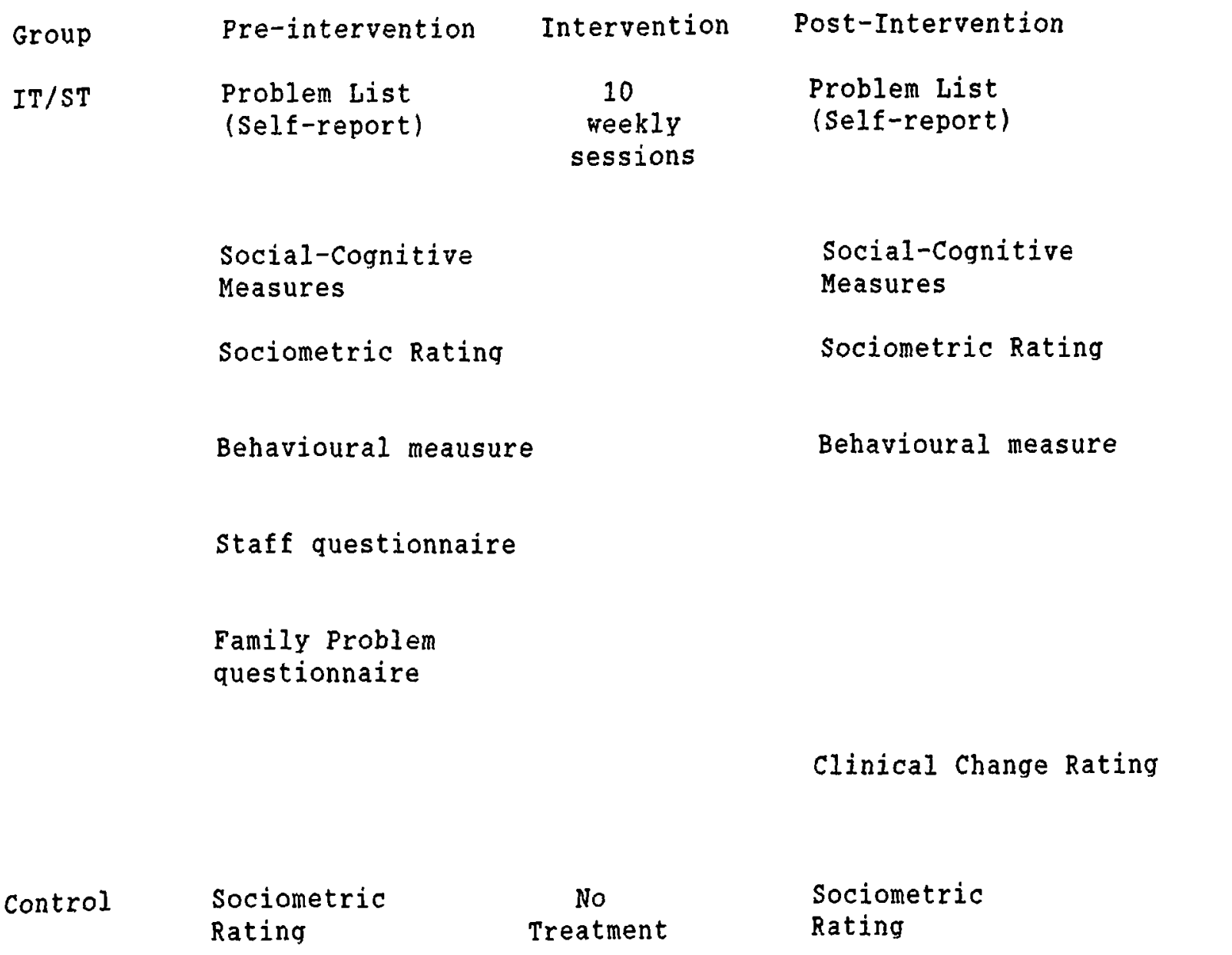


Assessment measures (Detail)

Assessments were completed by staff, families, peers, therapists and the clients themselves in order to obtain information on a wide range of situations and behaviours.

i) Pre intervention only.

a) The "Staff Questionnaire on Social Behaviour" (Spence, 1980) was used to yield diagnostic information on peer relationships, staff relationships and general social behaviour. Clients are rated on items such as "talks freely with peers" "initiates conversation with staff at appropriate times" and "facial expression is appropriate to situation".

b) The Family Problem Questionnaire (designed for this study, see Appendix 3) provided information on the family's view of the clients. Families were asked to assess the frequency and severity of any difficulties at home, (e.g. "Does $X$ have problems within the family? Are they frequent? Are they prolonged?") and also to rate clients on general social behaviour le.g ratings on eye contact, posture, response to questions).

ii) Pre and post intervention.

a) Self report questionnaire.

The full form of the Social Problem Checklist (Spence, 1980) was used. This comprises 37 questions about social situations such as "Do you take friends home often", "Do you feel awkward with new people", "Do you tease others". All questions required a "yes" or "no" answer. This questionnaire was used 
information as to whether a child was "neglected" (isolated or withdrawn) or "rejected" (antisocial) (Ladd and Asher, 1985). This differentiation was achieved by breaking down the questionnaire items into two categories.

b) Sociometric questionnaire.

A roster rating questionnaire, in which each student in the year group selected and rated five classmates from the class register, was used for screening and for assessment (pre and post).

c) The behavioural interview schedule.

In the Behavioural Interview Schedule the client is examined orally and the responses are recorded. The client is asked to say how he would deal with certain stressful social situations, e.q. needing to apologise to a teacher about something but feeling very nervous about it. There are five items scored on a scale of 0-1-2 using defined criteria as on a psychometric test. The instrument was piloted for reliability. Examiners were given training on the instrument prior to its use. The behavioural interview schedule is a behavioural measure.

\section{Sample content}

What do you usually do if you are talking to someone you don't know well and you run out of things to say? 
Sample response

"Tell them to do some talking."

Source of instrument

This instrument was designed for the present study (See Appendix 4). Van Hasselt et al. (1979) discuss the problems of quantifying social difficulty in behavioural terms. They suggest that using an interview technique may be effectve when used in conjunction with other strategies, though surprisingly few researchers have conducted interviews in social skill investigations with children.

d) The roletaking test.

The Role-taking Test is an assessment of social-cognitive functioning. Role-taking is described as the "ability of an individual to consider simultaneously his own point of view and that of others" (Van Hasselt et al., 1979). A form of the test for use with children was developed by Reardon et al., (1979). The test was modified for use with young adolescents in the present study. Eight pictures from the Rosenzweig Picture-Frustration Study ( Rosenzweig et al., 1948) were selected. In each of these pictures a child was shown in interaction with another child or an adult. The person on the left said something frustrating to the child in each picture (for example, "You didn't invite me to your birthday party"). This statement was printed in a "balloon" in the top part of the picture. Above the child on the right (with whom the subject was presumed to identify) was a second empty balloon. The subject did not however write a response in the empty balloon; the test was conducted interactively and an examiner recorded the subject's response, ie the subject's interpretation of how the child in the picture would deal with the frustrating situation. 
The subject was then asked how the person on the left would respond to that response, and this again was recorded. Thus having identified with a figure in the picture, the subject had to mentally switch roles. The response attributed to the person on the left was scored $0-1-2$ according to previously defined criteria of social appropriateness. The examiner was instructed to press for a reasonably elaborated response. The test was piloted for reliability and examiners were trained in its use. 


\section{Sample response}

"Would you like to push my marble out of the way?"

e) The social assertiveness test.

The Social Assertiveness Test was based on the responses attributed to the identification figure in the Roletaking Test. Responses were scored in a similar manner for social appropriateness using previously defined criteria. Like the Roletaking Test, the Social Assertiveness Test is a social-cognitive measure.

on introducing the pictures, the examiner says "I want you to tell me sensible ways that people in these pictures can stand up for their rights."

\section{Sample responses}

"I will just wait until you get off the swing".

"Sorry I'm late".

Assessment of scorer reliability on the clinical measures.

The Behavioural Interview Schedule, the Roletaking Test and the Social Assertiveness Test were together designated the Clinical Test Battery and record forms were desiqned for the use of administrators, who received training in the interactive use of this test battery (Appendix 4). When the results of five subjects on the whole of this battery were scored blind by two judges, the spearman Rho correlation was .59 for score totals on the pooled measures. Item by item on the pooled measures. the correspondence was .67 when the number of agreements was expressed as a function of the 
total number of items.

iii) Post intervention only.

Clinical change rating.

Therapists were asked to rate subjects at the end of the intervention for change on each of the following dimensions: self-expressive skills, otherenhancing skills, assertive skills and communication skills (Rinn and Markle, 1979). A number of items on each dimension were sampled and a five point rating scale was used (Appendix 7$)$.

Intervention: The training package.

Before the programme began subjects in the treatment groups were given a full explanation of what would be involved and had an opportunity to express their feelings about it. They also had the opportunity to meet one of the therapists for their group on an individual oasis. All subjects with one exception appeared to have good motivation for attending the group. Parental consent was obtained in each case. Parents were given full details of the project. In the first year of the project parents were visited personally by staff, but this was not possible in the second year owing to the constraints of industrial action. Training sessions for the two groups were held on a weekly basis. They lasted for an hour and a quarter. Soft drinks and biscuits were provided at the beginning of each group to create an informal atmosphere. There were ten sessions. The subjects were withdrawn from timetabled activities by prior arrangement with the staff concerned. A comfortable room with a pleasant atmosphere was used for the sessions. 
The general approach in the training sessions was similar to that described in Study I. At each session a specific aspect of social interaction was considered. In the early sessions there was a focus on the verbal and nonverbal microskills used in social interaction. This enabled clients to develop a conceptual structure and a "dictionary" of social skills. In the later sessions there was an emphasis on situations common to clients' experience to give them a chance to develop and apply their skills in "real life". Teaching, group discussion, modelling, role play and real life assignment setting were used. Assignment setting was given increased emphasis during the last four sessions. Subjects in the individualised treatment group were set "special assignments".

The formal structure of each session was similar to that used in Study I. After a "warm-up" activity, there was a brief cognitive input followed by extended role-play. In the last four sessions, there was an increasing emphasis on homework setting in the last part of the session. Therapists were provided with a manual explaining the aims and objectives of the course and setting out session plans (Appendix 1).

In order to provide a more detailed illustration of the material, the introductory section of the course manual is included here, together with (a) the content of the first session (b) session formats for the remaining sessions. There are specific instructions which are given only to therapists conducting the individual training sessions. This variation in instructions occurs in the introductory section and in the last four sessions of the intervention. These instructions relate to the setting of homework assignments and the behavioural preparation for those assignments. Sample instructions from session 7 are included, to illustrate the 
contrasting features of the standardised condition and the individualised condition.

\section{Course Manual}

(Instructions underlined are issued to therapists for Individualised Training Groups only. For Sessions Seven to Ten, there are separate instructions for the two training conditions.)

Aims of Course

1. By creating an atmosphere of trust the therapists will provide an experience of positive social interaction within a structured setting.

2. Procedures for dealing with everyday social situations will be studied, developed and practised using modelling, role-play and feedback. The therapists will control the feedback process but will bear in mind that direct feedback is a powerful reinforcer.

3. The training procedures will encourage clients to shift the focus of concern from self to others, attending more to others' feelings than their own, though appropriate assertion of their own rights and feelings will form part of the training. 
4. Clients will learn to structure situations to enable them to practise newly acquired social skills and consolidate those that are already part of their repertoire.

5. Clients will learn to be more aware of their anxieties in social situations, eq when authority fiqures or a deqree of conflict is involved.

6. Clients will learn to generalise the skills learned and perfected in the group to other situations. "Special assignments".will be set to enable practice in "real life" situations to occur. These special assignments will be geared to the specific needs of individuals.

7. The therapists will be provided with social skills assessment profiles on each of the youngsters and will make conscious efforts to provide appropriate training to individuals in accordance with their special needs, so lona as the constraints of working. in a group context are taken into account.

Format of sessions

1. Getting the group together

2. Warm-up (loosening up activity)

3. Main work (discussion / roleplay / homework setting)

4. Closure (eg favourite game) 
Main skills to be studied

Coping with teasing

Negotiating

Being left out

Asking permission from an adult

Joining a group

Coping with bullying

Talking to a strancer

Being wronaly accused

Arranqing to meet friends

Helping others to solve problems

Maintaining conversation

standing up for your rights

Introducing yourself

Paying a compliment

Giving directions

Apologising

Understanding the feelings of others

Dealing with someone else's anger

Dealing with fear

Avoiding trouble with others

Dealing with contradictory messages

Getting ready for a difficult conversation
Answering a complaint

Setting a goal

Gathering information

Dealing with group pressure

standing up for a friend

Dealing with embarassment

Concentrating on a task

Makinq a complaint

Keepinq out of fights

Using self-control 
Games and Warm-ups

$\begin{array}{ll}\text { Name Game } & \text { Echo circle } \\ \text { Pass the cat } & \text { Blah-gab } \\ \text { Luckily/unluckily } & \text { Reflecting } \\ \text { Eye-wink murder } & \text { Knots } \\ \text { Passing the message } & \text { Blind partners } \\ \text { Sculpture of emotion } & \text { In the manner of the word } \\ \text { Expression of emotion } & \text { Physical proximity } \\ \text { Looking meditation } & \text { Leaning out circle } \\ \text { Meditation } & \text { Zip boing } \\ \text { Saying no } & \text { Yes/no }\end{array}$

Session one

1. The Name Game

Group members sit in a circle. One member says his name. The next member repeats that person's name and then says his own. This continues around the circle so that the seventh person has to say the names of the six previous people. When the circle is completed, start in a new place and go the other way round, but this time each person puts a descriptive adjective in front of his name and repeats the other names and adjectives, eg "Lazy Joe, Tough Tom, Quiet Joanne". 
The group sit on the floor in a circle. Someone mimes holding a cat and then passes the imaginary cat to the person on one side of him, and so on round the circle. Continue with other objects, eg chewing gum, snake, length of elastic, bucket of slime.

\section{True story}

Split group into pairs. A describes a true incident from his experience. $B$ repeats this with $A$ 's inflection and emphasis. A then tells the story again but in a different role - eq an absent minded old woman. Encourage partners to discuss the task afterwards and to give each other positive feedback.

\section{Set the scene}

Briefly outline the aims of the aroup and what you expect of group members. Deal with any questions that come up. 
Aims of session

1. Introduce group members to each other

2. Create cohesiveness

3. Beginnings of social interaction within the group

4. Introduce mime to draw attention to hand movements

5. Provide enjoyable experience

6. Enqender motivation

7. Promote self-disclosure

8. Promote lively verbal exchange

9. Establish relationship with group

10. Set boundaries 


\section{Session Seven \\ Individualised Training Groups}

1. Our Day

2. Eye Contact Greeting

3. Special Assignments - Introduction of Concept

Introduce notion of individual "Special Assignments" over the next three weeks. Allocate individuals to sessions. Explain purpose of assignments, ie practice in real life situations.

4. Roleplays

Select themes geared to needs of individuals undertaking assigments this week, eg negotiating, paying a compliment, understanding the feelings of others. Highlight points that will be useful to those doing assignments.

\section{Special Assignments - Setting Tasks}

Discuss tasks, which should be tailored to remediation of the deficits of the individual concerned. Tasks should be practicable and neither too easy nor too difficult. Opportunities for practice (ie carrying out the tasks in real life) should be plentiful. Put details of agreed task on white card as reminder. 
6. Game

Aims of session

1. Introduce concept of Special Assignments

2. Assign real-life tasks appropriate to individual need

3. Development of relevant skills through roleplay

4. Promote generalisation 
1. Our Day

2. Warm Up

Therapists select suitable activity.

3. Roleplays

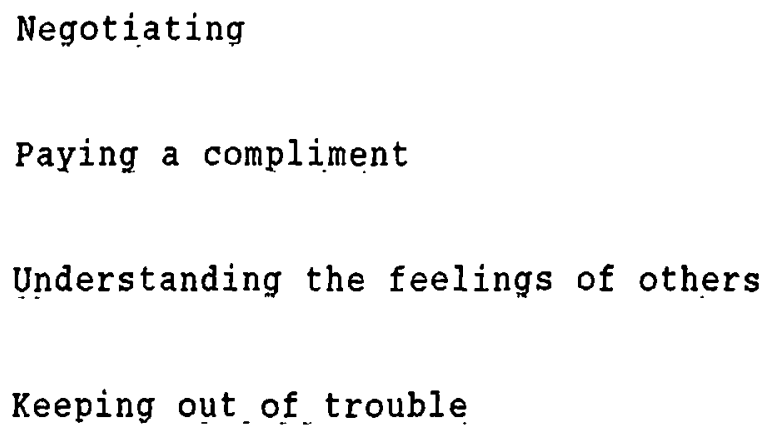

4. Homework Setting

Select a simple task associated with the roleplays, e.g. paying someone a compliment. Ask each member of the group to carry this out in real life and report back next time. The task should be neither too easy nor too difficult and each client should have plentiful opportunities for carrying it out. Give each client, by way of a reminder, a white card on which details of the task are noted.

5. Eye Wink Murder 
Aims of session

1. Continue to develop more complex skills through roleplay

2. Offer real life opportunities to practise the skills acquired

3. Promote generalisation

Session Formats for sessions $2-10$

\section{Session Two}

1. The Name Game

2. Looking Meditation

3. Expression and Recognition of Emotion

4. Friend across the street

5. Luckily/unluckily

\section{Session Three}

1. Introductory Exercise

2. Sound Circle Echo

3. Sound Circle

4. Questioning Numbers

5. Eye Contact Greeting

6. Physical Proximity - Roleplay

7. Eye Contact Murder 
Session Four

1. Leaning out Circle

2. Blah Gab

3. Verbal Mirroring

4. Verbal Reflecting

5. Game

\section{Session Five}

1. Introductory Activity

2. Saying No

3. Roleplays
i) Joining a Group
ii) standing up for your rights

4. Game

Session Six

1. Our Day

2. Roleplays

3. i) Dealing with bullyina

ii) Being wrongly accused

4. Game 


\section{Session Seven}
i) Individualised Traininq
ii) Standardised Training
1 O:r Day
1. Our Day
2. Eye Contact Greeting
2. Warm Up
3. Special Assiqnments
3. Roisolays
4. Roleplays
4. Homework Setting
5. Special Assianments-
5. Eye Wink Murder Setting Tasks

\section{Session Eight}

Individualised Trainina

Standardised Training

1. Our Day

1. Warm Up

2. Warm Up

2. Homework Assignment Feedback

3. Special Assiqnment Feedback

3. Roleplays

4. Roleplay

4. Homework Setting

5. Special Assianment Settina

5. Game

6. Game 
Session Nine

Individualised Traininq

Format as for

Session Eight

\section{Session Ten}

Individualised Trainina

1. Our Day

2. Future Plans

3. Special Assignment Feedback

4. Training for Generalisation

5. Role Play

6. Video Playback

7. Game
Standardised Trainina
1. Harm Up
2. Homework Assignment Feedback
3. Roleplays
4. Homework Setting
5. Game

Standardised Training

1. Warm Up

2. Homework Feedback

3. Review Achievements

4. Roleplay

5. Video Playback

6. Game 


\section{Results}

The results were analysed in two stages. In the second staqe, new hypotheses were explored. Consequently, for the sake of clarity, the results are set out in two sections.

\section{Section One}

i) Main outcome measures.

Means and standard deviations of the outcome measures "Sociometry" and "Self-report" are presented in Table 1, broken down by Training Condition. The same information is presented in Graphs 1 and 2. 
Table 1.

Means and Standard Deviations on Measures of Sociometry and Self-Report

Time of Assessment

and

Behavioural Measure

Pretest

Sociometry

Sociometry

Self-report

self-report

Posttest

Sociometry

Sociometry

Self-report

Self-report
Individualised

Standardised

Individualised

Standardised
N

Mean

SD

Condition 


\section{Experiment 2.}

Graphs 3 and 4 : Significant Changes for Therapist Pair A
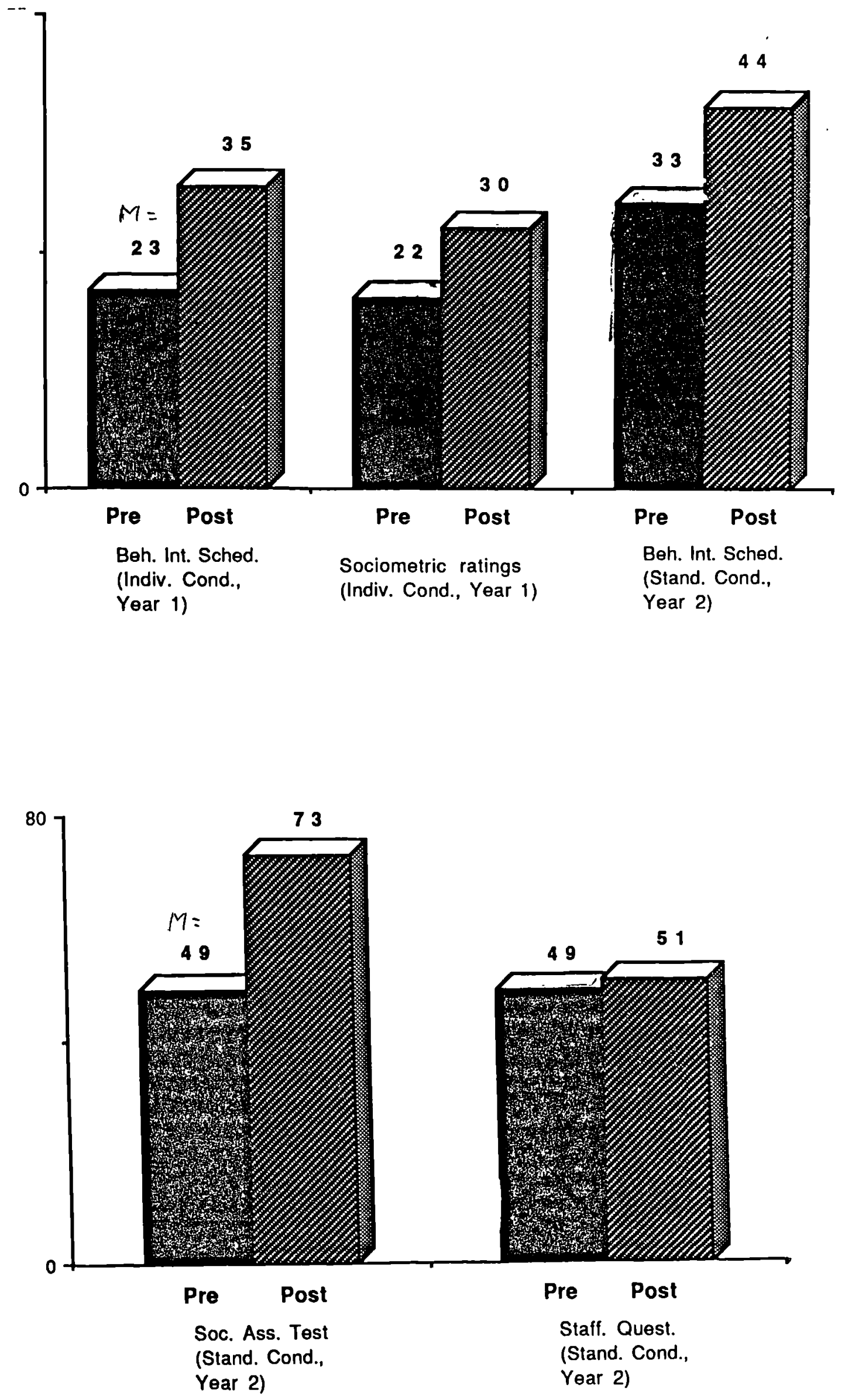
Experiment 2.

Graph 5 : Significant Change for Therapist Pair B

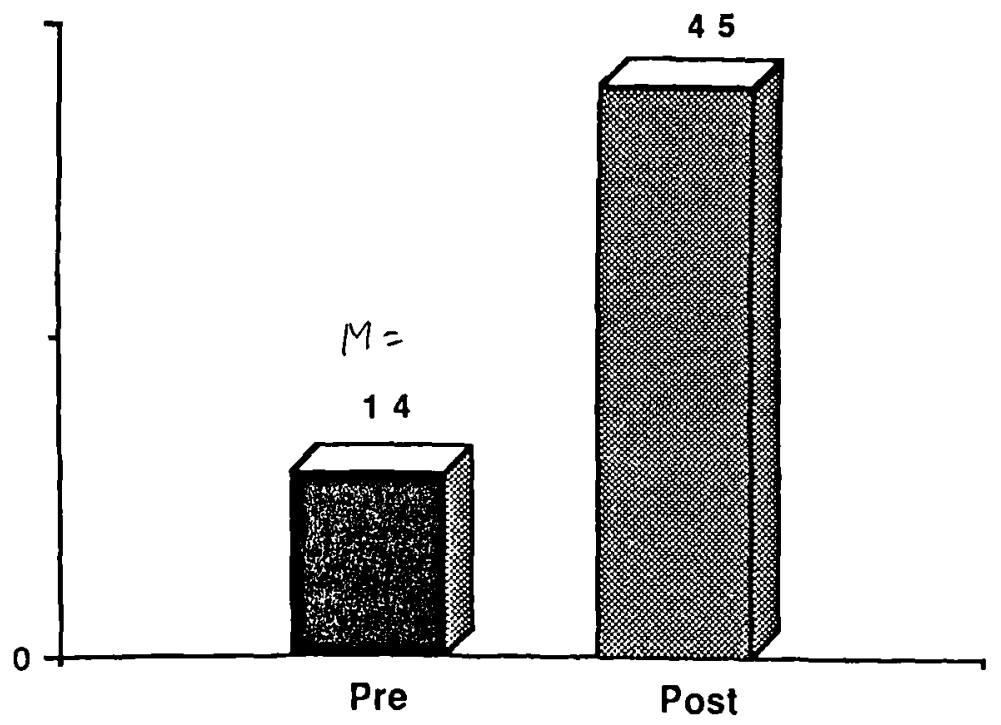

Sociometric Ratings (Individualised Condition, Year 2) 
ii) Group comparison pre intervention.

The Individualised Training subjects were compared with the Standardised Training subjects collapsing both years. Prior to intervention, the difference between scores obtained by Individualised Training subjects and those obtained by the standardised Training subjects on the self-report questionnaire approached siqnificance $(t=1.05$, df $=26, p=.095,2-$ tailed). The Individualised Training subjects appeared to have slightly greater social difficulty than the Standardised Training subjects. On the sociometry measure, the difference between the two sets of subjects in the pre condition was not significant.

It appeared that the Family Problem Score was slightly greater for the Individualised Trainina subjects than for standardised Training subjects. However as in the case of the self-report questionnaire, the finding was not statistically significant. 
iii) Group comparison: Treatments.

SPSSX Manova (Repeated Measures) was used to assess the effect of the independent variables Group (i,e Training Condition). Time (Pre vs. Post) and Group/Time Interaction on the pooled dependent measures, self-report and sociometric ratina. These two measures were pooled in order to obtain an overall estimate of the effectiveness of the training. There is a provision in Manova for the pooling of measures. The effect of the variable Time was significant $(F=4.8, \mathrm{df}=2.22, \mathrm{p}=.019)$ and this would suggest that overall there was a reduction in social difficulty as predicted for the two aroups by the end of training. The effect of Group/Time Interaction was not significant. It would appear that the majority of the variance was explained by the main effect.

SPSSX Ancova was used to check the effect of the independent variables Group (i.e Trainina Condition), Problem (Rejected/Overassertive vs. Neglected/Underassertive), and Gender on the pooled dependent measures Sociometric Rating (post) and Self-report (post). Sociometric Rating (pre) and Self-report (pre) were co-varied out. Hence it appeared that the outcome on this pair of measures was not influenced by subjects' type of problem, gender, or type of training received.

The Role-taking Test was analysed for possible differentiation between outcomes in the two training conditions. Again, usina Manova, no Group/Time Interaction was found. However, change scores were computed for this measure. All subjects receiving social skills training were included in the analysis. Subiects receiving Individualised Training made siqnificantly greater gains than subiects receiving standardised Training $(t=1.97$, df $=$ $13, p=.035,1$-tailed). 
The Individualised Trainina subjects also showed significantly qreater signs of clinical gain on the clinical Change Rating in Year $1(U=1, d f=$ 13, $p=.035,1$-tailed; Mann-Whitney).

iv) Group Comparison: (Traininq vs. Control Conditions)

Comparison of the means of the control group with the social skills training groups is aiven in Table 2. (Also see Graph 1.) owing to a teachers' strike it was not possible to obtain control data on the selfreport questionnaire. There was no evidence that the social skills training aroups made more aains than the control aroup on the sociometric rating. There was no significant change from pre to post in the control group.

In the second year, there was no significant change in the control group on the teacher questionnaire. However Therapist Pair A produced significant change on this measure in the standardised Training condition $(\mathrm{T}=0, \mathrm{df}=$ $6, p<.05,1$-tailed; wilcoxon) . 
Table 2

Means and Standard Deviations of Individual, Standard and Control Groups on Sociometric Measures qiven Pretest and Posttest

Time of Assessment

Group

$\mathrm{N}$

Mean

SD

Pretest

Indiv. Train. 14

4.29

6.66

Posttest

Stand. Train.

11

6.09

5.2

Contral

14

7.14

4.96

Indiv. Train.

14

6.36

5.97

Stand. Train.

11

6.91

5.63

Control

14

10.36

8.03

v) Change from pre to post.

On the self-report questionnaire there was a significant improvement from the "pre" condition to the "post" condition $1 t=2.31, d f=14, p=.019,1-$ tailed). Both qroups from both years were collapsed for this analysis. There was no corresponding significant improvement in sociometric status for the combined aroups, but the Individualised Training qroup taken alone approached siqnificant chanqe $(z=-1.6, d f=13, p=.05,1$ tailed, Wilcoxon). The means are presented in Graph 2 or comparison. 
Therapist Pair A produced significant change for the Individualised Training group in Year 1 on the Behavioural Interview Schedule $(T=0, d f$ $=6, p<.05,1$ tailed, Wilcoxon $)$ and on sociometric status $(T=3, d f=6$, $p(.05,1$ tailed, Wilcoxon) and for the Standardised Training group in Year 2 on the Behavioural Interview Schedule $(T=0, d f=6, p<.051$ tailed, Wilcoxon), the Social Assertiveness Test $(\mathrm{T}=0, \mathrm{df}=6, \mathrm{p}<.05,1$ tailed, Wilcoxon), and the staff Questionnaire ( $\mathrm{T}=0, \mathrm{df}=6, \mathrm{p}<.05,1$ tailed, Wilcoxon). Therapist Pair B produced significant change for the Individualised Training group in Year 2 on the sociometric questionnaire ( $\mathrm{d}>0, \mathrm{df}=4, \mathrm{p}<.05,1$ tailed, Wilcoxon). Details of client changes from Pre to Post in all years and both conditions are given in Appendix 10 . Details of therapist effects are given in Graphs 3, 4, and 5 .

vi) Therapist effects (Role-taking).

Using change scores on all subjects, the change produced on the Role-taking Test by Therapist Group A was of significantly greater magnitude than that produced by Therapist Pair $B(t=1.97$, df $=13, \mathrm{p}<.05,2$ tailed).

vii) Type of problem.

There was no significant difference in chanqe score outcome on the Roletaking test attributable to "Type of problem", i.e "rejected"/overassertive vs. "neglected"/underassertive. 
viii) Single case designs.

Four sets of findings on Individualised Training subjects $(2$ male, 2 female) are reported in Appendix 2, giving full psychometric information. Each report provides "case" information, an assessment profile, a qualitative interpretation of psychometric instruments, and an evaluative comment. The assessment profile provides quantitative information pre and post on the following indices; sociometry, teacher nomination, self-report, social assertiveness, role-taking, behavioural interview, staff questionnaire, Bristol Social Adjustment Guide, and family questionnaire.

Client 1, "John", showed improvement on three indices (sociometry, teacher nomination, and behavioural interview schedule), deterioration on one (self report) and no chande on two (social assertiveness and role-taking). On the Clinical Change Rating, he showed positive change on five items and was unchanged on four.

For illustration full details of the case study on John are given below.

Case report. (based on interview post-training)

John had very mixed feelings about the group. His comment was "It's better than doing maths." He feels that he has made more friends since being in the group and thinks that the group helped him with this.

He said that he was referred to the group because "I am weird". He said that he needs to be weird because the friends he has expect it of him. When John as asked when he would be able to drop this weird bit of himself, his reply was "when I leave school". He talked about the possibility of 
his family moving to a different area, and said that if he moved to another school he might be able to drop the "weird bit".

John is the youngest of a family of five children but does not feel that he is treated like the baby of the family. Unlike June, John had to compete very hard in this large family to get his needs met.

John talked sensibly and appeared to have a good understanding of his difficuities and how they might be detrimental to his future.

He told the therapist that he had a fight that morning. They talked about why this happened in some detail. He said that his cat died and he was quite sure that it had been poisoned by someone. He felt both angry and upset about this and really did not know how to handle it. He did know that the boy whom he attacked had not in fact harmed his cat; he had just teased him a bit that morning. He was able to see that he had unleashed his angry feelings about the harm done to his cat upon this boy.

John seems to understand that he loses control and expresses his anger towards the wrong people for the wrong reasons.

John was asked he would like a further group experience of a similar kind. He said that he would definitely come back to the same group with the same group leaders, but that he would not want to start again with another group.

John clearly did need ongoing help and it was felt that it might be appropriate to offer him the booster group to see if he will accept it.

Prior to seeing John I had a brief discussion with the Headmaster, who 
informed me that he had referred John to the Educational Psychologists because of his outbursts. He felt that the school had exhausted all the various approaches with him.

As John had not been told about this the therapist did not discuss it with him. There was clearly a need for further work and it might be appropriate for the Educational Psycholoqists to look at what kind of programme might be set up within the school to help him.

John: Assessment profile.

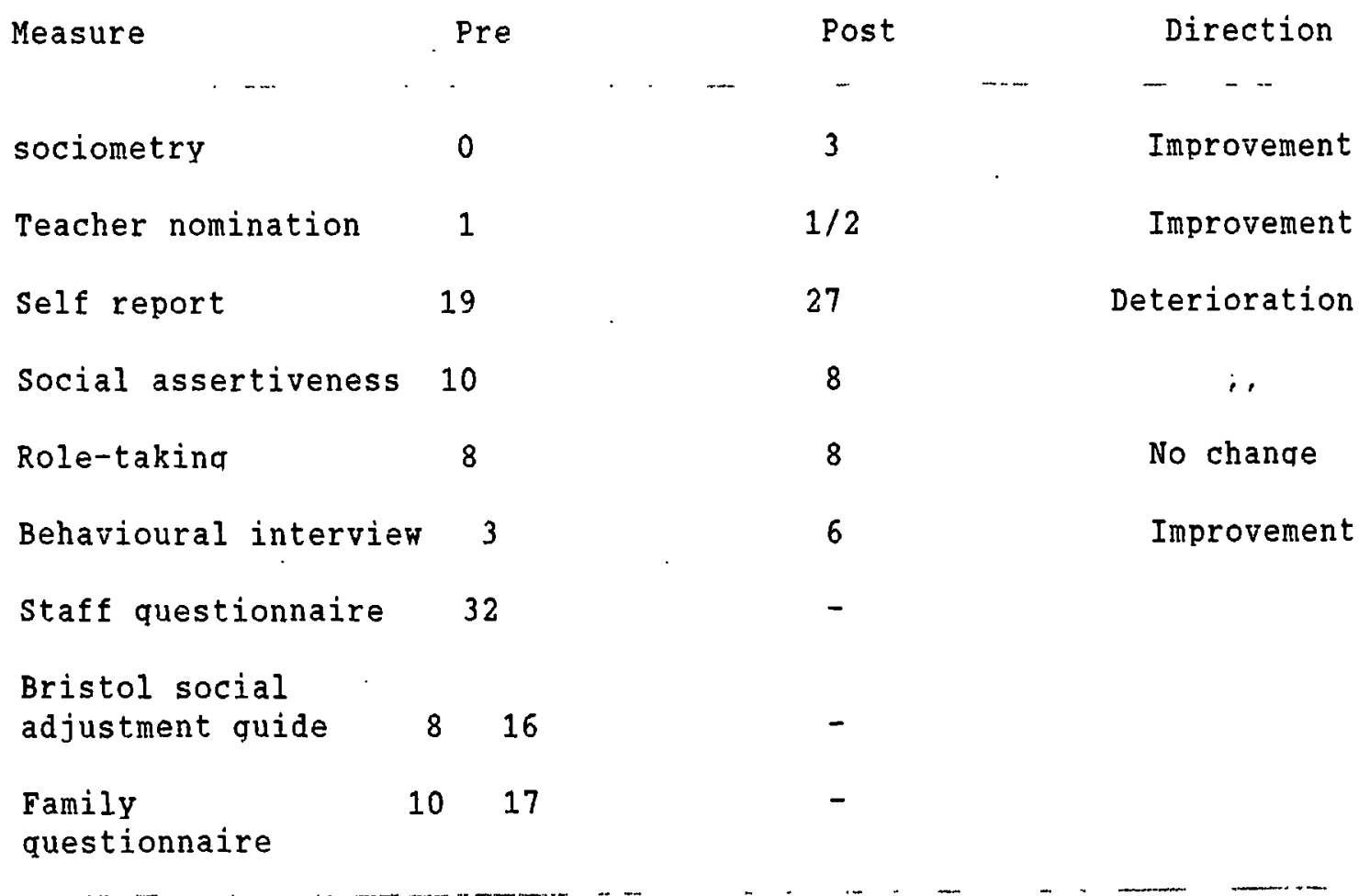


Self report questionnaire.

John underlined the item: (Do you) "Find it hard to keep your temper when an adult won't let you do something". He ringed 6 "antisocial" items and 13 "unassertive" items.

Family questionnaire.

John came from a "traditional" two parent family. The family were quite aware of his problems in making and maintaining friendships and in controlling his behaviour. There were frequent arguments at home with all family members. It was felt that he had difficulty relating appropriately to staff and was teased or bullied by peers at times. He was judged to have severe difficulty with eye contact and moderate difficulty with facial expression, fidqeting and fluency of speech.

Clininal change rating.

On the Clinical Change Rating, John was felt to have improved in expression of feeling, expression of opinion, positive statements about others, praising others, disagreeing with others, and conversation skills. He was felt to be unchanged in respect of avoiding disputes, refusing unreasonable requests, positive statements about self, and accepting compliments.

Evaluative comment.

Prior to treatment, John was perceived by staff as a rejected, antisocial child (this is the sianificance of the teacher rating). From the staff questionnaire, it is clear that the staff believed that he had a good deal 
of difficulty in social interaction. The Bristol Social Adjustment Guide indicated that he had a problem of overreaction. The Family Questionnaire indicated that he had a qood deal of difficulty both in terms of problem severity and "micro-skills". He was clearly quite isolated from the peer group.

Post treatment he showed a very slight improvement in sociometric status. The teacher rating changed from 1 (antisocial) to $1 / 2$ (antisocial/unassertive). This would suggest he might have been overreacting less. The apparent deterioration in self report may have been due to a more accurate appraisal of his problems. There is evidence from the Behavioural Interview Schedule that he was perhaps able to control his behaviour rather better, though the aloomy prognosis of the Head would appear to cast some doubt on this point. John's comments at interview and the Clinical Chanqe Rating do indicate that at least moderate improvement occurred, even though this was clearly not sufficient to keep him out of trouble.

Consequently the psychometric indicators in conjunction with the clinical interview strongly suquest that a clinical improvement occurred which statistical analysis miaht not have detected. This was an extremely "problematic" client, and a "miracle" cure is not claimed, but it is valuable to know that the treatment package had some relevance to his needs. 
Client 2, "June", was assessed by teachers as unassertive prior to training. Her sociometric status suggested that she was isolated. In interview she admitted being "left out". The family assessment suggested the presence of moderate social difficulty.

She said that she had enjoyed the qroup. She was aware of her presenting problem of isolation. She said that there had been an improvement in that she now had more friends. She was the younqest of five children, the only girl. Her mother suffered from asthma and miaraine. It was hypothesised that as a result of her position in the family June had not been required to assert herself very viqorously in her first decade in order to obtain attention. With some individual attention to her social skills, June had no difficulty in altering her pattern of functioning with her peers. June showed improvement on four indices (sociometry, self-report, roletaking and behavioural interview schedule) and no change on one (social assertiveness). On the Clinical Chanqe Rating, she made substantial gains across each of the three main areas - self-expressive skills, otherenhancing skills, and communication skills. 
Client 3, "Ian", was clearly "problematic" prior to treatment. His sociometric status was low and he was assessed by staff to be unassertive. Questionnaire outcomes from both staff and the family suqgested substantial social skill deficits. Ian was seen as socially isolated and unresponsive, and he appeared to have difficulty in initiating and maintaining contact with other children. The appeared to be difficulty in his family relationships, particularly with his two younger brothers. Ian's mother had changed partners twice during his life time.

After the completion of training, Ian felt that the aroup had helped him in that he was less shy than he was. He felt that he still required further help with his problem.

Ian showed improvement on three indices (sociometry, self-report, and behavioural interview schedule), deterioration on one (social assertiveness), and no chanqe on one (role-taking). On the clinical change rating he reaistered improvement across the three main areas.

client 4, "Sharon", was somewhat resentful as the result of having a very attractive older sister. She felt that she could not bring a boy friend home as he would inevitably prefer her sister. She had many clashes with her father who was something of an authoritarian. Her mother was physically unwell so she found it difficult to arque with her.

Sharon was identified by teachers as "antisocial". Her initial score on the behavioural interview schedule was low, suggesting that her behaviour was uncontrolled. Questionnaire data and behavioural observation identified a tendency to overreact and severe behavioural difficulty within the family. 
Sharon felt that she derived benefit from the group, and made more friends as a result of it. There was an improvement on the behavioural interview scale and the clinical change rating was extremely positive. It was clear from the follow-up session that coanitive gains had been made, as sharon was able to describe ways in which she had been able to apply the suggestions made within the aroup. It seems likely that sharon gained considerably in confidence and in social competence as a result of the aroup.

ix) Summary of findinas.

Significant findings from Results, section one are summarised in Table 3. 
Table 3 .

Summary of Significant Findings ( Section one).

Therapist Year Group Measure

Pair

$\mathrm{A}+\mathrm{B} \quad \mathrm{IT}>\mathrm{ST} \quad$ Roletaking

$\begin{array}{llll}A+B & 1 & \text { IT }>\text { ST } & \text { Change rating }\end{array}$

$A+B \quad 1+2 \quad I T+S T$ Self-report

+ sociometry *

$A+B \quad 1+2 \quad I T+S T$ Self-report *

A+B $1+2$ IT Sociometry *

A 2

ST Staff rating *

A 1

IT Behavioural

interview *
A
1
IT
Sociometry *
A
2
ST
Behavioural
interview *
A
2
ST
Social
assertiveness *
B
2
IT
Sociometry *
A > B
$1+2$
$I T+S T$
Roletaking
$I T$ = Individualised Training
$S T=$ Standardised Training 


\section{Results}

\section{Section Two}

In this section the results on three measures are of principal interest: the role-takina test (a social-cocnitive test, not a test of role-play as its name might suggest), the sociometry assessment procedure, and the selfreport questionnaire. This time the roletaking test was included in the main analysis as its discriminatory power had been established by the results of the initial exploratory analysis. Subjects with missing scores were omitted. One further subject, whose sociometry initial score was 25 while the rest were in the 0-5 ranqe, was excluded as an "outlier". The remaining 22 subjects were included in the analysis.

The correlation between measures and the relative effectiveness of therapist pairs are also examined in some detail. The significant difference between therapists obtained on the chanqe score in the first analysis of results led to an increased interest in therapist differences, as these would have a bearing on the final interpretation of the findings.

First, the significance of difference between pre and post scores was calculated using $t$ tests for each of the three measures when all 22 subjects were "pooled" (Table 1). On the role-taking test the difference between pre and post scores was siqnificant at the .01 level ( $t=-2.59$, df $=21, p=.008,1$ tailed). On the self-report questionnaire the difference between pre and post scores was also significant at the .01 level $1 t=$ 2.84, $\mathrm{df}=21, \mathrm{p}=.005)$. On each of these measures the sianificant change shown was indicative of improvement in social skills. On the sociometric measure, improvement was attained but just failed to reach sianificance. 
Table 1

Difference between Pre and Post Scores for Behavioural Measures with All Subjects Pooled

Behavioural Measure

and

Time of assessment

Mean

SD

t

p

(1 tailed)

Roletaking

$\begin{array}{lllll}\text { Pre } & 8.0 & 1.63 & & \\ & & & & \\ & & & -2.59 & .008 \\ \text { Post } & 9.22 & 1.79 & & \end{array}$

Sociometry

$\begin{array}{lllll}\text { Pre } & 4.45 & 4.55 & & \\ & & & & \\ & & & & \\ & & & & \\ \text { Post } & 6.18 & 5.20 & & \end{array}$

Self-Report

Pre

31.0

8.89

$>$

2.84

.005

Post

$26.6818 \quad 8.88$

Note: $N=22$ 
Next, one-tail t-tests were used to assess the difference between pre and post scores on each of the three measures in the Individualised Training Condition (Table 2). All subjects were pooled for this analysis. On the sociometric measure, the difference between pre and post was significant at the .05 level $(t=-2.23, d f=11, p=.02)$. This was an interesting finding, as there was no sianificant difference on this variable when subjects were pooled in Table 1 . Siqnificant improvement was also shown on the roletaking test $(t=-1.97, d f=11, p=.04)$ and self-report $(t=$ 1.79, $\mathrm{df}=11, \mathrm{p}=.05)$. The improvement on all three measures was in the predicted direction. 
Table 2

Difference between Pre and Post Scores on Three Measures for Subjects Treated under the Individualised Training Condition

Behavioural Measure

and

Time of Assessment

Mean

$S D$

$t$

$d f$

p

(1 tailed)

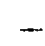

Roletaking

Pre

8.5

1.24

> $-1.97 \quad 11$

.04

Post

9.5

1.50

Sociometry

Pre $\quad 2.91 \quad 3.11$

$\begin{array}{llll}> & -2.23 & 11 & .02\end{array}$

$\begin{array}{lll}\text { Post } & 5.83 & 4.85\end{array}$

Self-report

Pre

32.83

8.22

$>$

1.79

11

.05

Post

29.50

6.30

Note: $\mathrm{N}=12$ 
Thirdly, t-tests were used to assess the difference between pre and post scores on each of the three measures in the ST condition (Table 3). Significant improvement in the predicted direction was shown on the selfreport measure $(t=2.16$. df $=9, p=.03$ ). On the roletakina test there was improvement which approached sianificance; there was no evidence of significant change on the sociometric measure, though again the change was in the direction of improvement. 
Table 3

Differences between Pre and Post on Three Measures for Subjects Treated Using the Standard Condition

Behavioural Measure

and

Time of Assessment

Mean

$S D$

$\mathrm{t}$

df

$p$

(1 tailed)

Roletaking

Pre

7.4

1.89

$>\quad-1.72$

9

.06

Post

8.9

2.13

Sociometry

pre

6.3

5.43

$>\quad-.16$

9

NS

Post

6.6

5.83

Self-report

Pre

28.8

9.60

$>2.16$

9

.03

Post

23.3

10.60

Note: $\mathrm{N}=10$ 
Fourthly (Table 4), t-tests were used to assess the difference in change score (ie difference in deqree of change from pre to post) between the IT condition $(\mathrm{n}=12)$ and the ST condition $(\mathrm{n}=10)$. On the role-taking measure significant improvement was shown in the predicted direction $(t=-2.06, d f=20, p=.03)$. No sianificant difference in degree of change was shown on sociometry or self-report.

Table 4

Difference between Individualised and Standardised Traininq as Indicated by Chanqe Scores from Pre to Post

Behavioural Measure Mean

and Change SD $t$ df $p(1$ tailed $)$

Condition Score

Roletaking

Individual $\quad 1.5 \quad 1.31$

$-2.06 \quad 20 \quad .03$

$\begin{array}{lll}\text { Standard } & 2.7 & 1.41\end{array}$

Sociometry

$\begin{array}{lll}\text { Individual } & 3.41 & 4.12\end{array}$

$>\quad-.9420 \quad$ NS

$\begin{array}{lll}\text { Standard } & 4.9 & 3.07\end{array}$

Self-report

\begin{tabular}{|c|c|c|c|c|}
\hline \multirow[t]{2}{*}{ Individual } & 5.16 & 4.95 & & \\
\hline & & $>$ & -.69 & 20 \\
\hline Standard & 6.9 & 6.75 & & \\
\hline
\end{tabular}


Fifthly, t-tests were used to calculate differences in change scores (differences in the deqree of change from ore to post) between the two pairs of therapists for each of the three measures (Table 5). The two pairs of therapists did not differ significantly in their change scores.

Table 5

Difference between Therapist Pair $A$ and Therapist Pair $B$ as Indicated by Change'Scores from Pre to Post

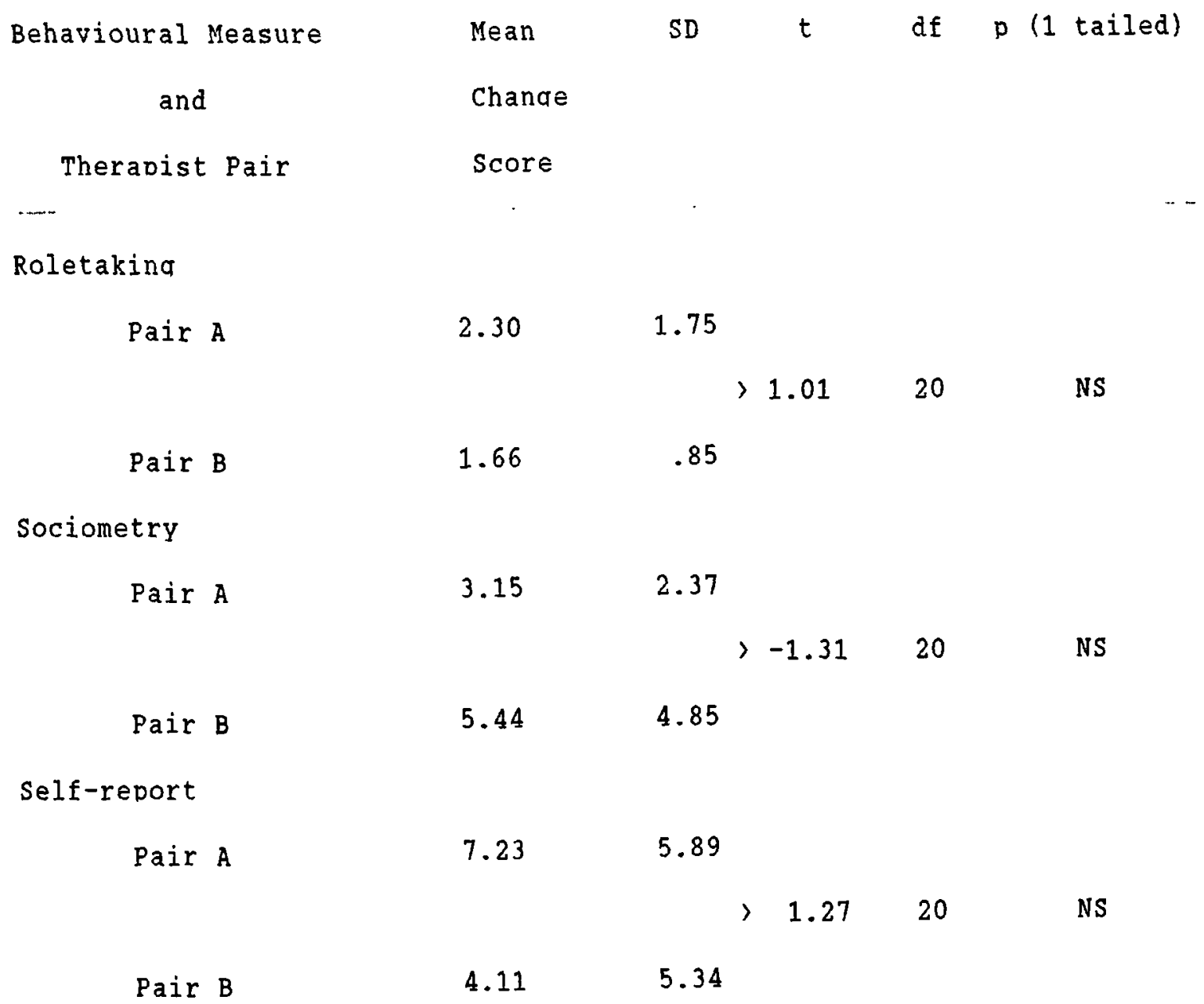


In the sixth analysis, t-tests were used to assess the difference from pre to post on the three measures for theradist pair A (Table $6, n=13$ ). Significant improvement in the predicted direction was shown on the roletaking measure $(t=-3.98, d f=12, D=.001)$ and on self-report $(t=2.11$, $d f=12, p=.031$. There was chanqe in a positive direction on sociometry but technical sianificance was not quite achieved. All subjects were used for this analysis.

Table 6

Difference between Pre and Post Scores for Therapist Pair A on Three Measures

Behavioural Measure

and

Time of Assessment

Mean

SD

$t$

df $p$ (1 tailed)

Roletakina

Pre

7.38

1.60

$>\quad-3.98 \quad 12$

.001

Post $\quad 9.53 \quad 1.33$

Sociometry

Pre $\quad 4.07 \quad 4.05$

$-1.58 \quad 12 \quad 07$

$\begin{array}{lll}\text { Post } & 5.69 & 5.39\end{array}$

Self-report
Pre
30.38
8.09

> $2.11 \quad 12$

.03

Post

25.61

5.83 
Finallv, the differences between pre and post were assessed for Therapist Pair $B$ on each of the three measures (Table 7). Siqnificant improvement in the predicted direction occurred only on self-report $(t=1.93$, df $=8, p$ $=.04)$. Improvements in the predicted direction did occur on roletaking and sociometry but siqnificance was not attained. There was therefore more limited evidence for the effectiveness of Therapist Pair B. Aqain both IT and ST subjects were used ( $n=9)$, excluding cases with missing scores.

Tạble 7

Differences between Pre and Post Scores on Three Measures for Therapist Pair B

Behavioural Measure

and

Time of assessment

Mean

SD

$t$

$d f$

p (1-tailed)

Roletaking

Pre

$8.88 \quad 1.26$

> $\quad .17$

8

NS

Post

$8.77 \quad 2.33$

Sociometry

Pre

$5.00 \quad 5.40$

$\begin{array}{lll}> & -.78 \quad 8\end{array}$

NS

Post

$6.88 \quad 5.15$

Self-report

Pre

$31.88 \quad 10.38$

$\begin{array}{llll}> & 1.93 \quad 8 & .04\end{array}$

$\begin{array}{lll}\text { Post } & 28.22 \quad 12.31\end{array}$ 
For interest, correlations were run to obtain some idea of test-retest reliability and intercorrelation between measures. There is some evidence of test-retest reliability for sociometry $(r=.42)$ and self-report $(r=.68)$. There was no sianificant intercorrelation between measures.

Table 8

Test Retest Reliability and Intercorrelation between Measures

Posttest

$\begin{array}{cccc} & \text { Roletaking } & \text { Sociometry } & \text { Self-report } \\ \text { Roletaking } & .16 & .01 & -.10\end{array}$

Pretest Sociometry $\quad .01$

$.42-.28$

Self-report $\quad-.13$

$-.07$

.68 
Results, Section 2

Summary of findings : outcome broken down by Training Condition, Therapist, and Measure

Type of analysis

Significant results

Implications

Pretest vs posttest

(AII subjects pooled)

Pretest vs posttest

(IT only)

Pretest vs posttest

(ST only)

Change score

(IT VS. ST)

Pretest vs posttest

(Therapist pair A)

Pretest vs posttest

(Therapist pair B)

\author{
Roletakina (.01 level) Sociometry \\ Self-report $(.01, \ldots)$ less sensitive \\ to immediate \\ change
}

\begin{tabular}{|c|c|c|c|}
\hline $\begin{array}{l}\text { Roletaking } \\
\text { Self-report } \\
\text { Sociometry }\end{array}$ & $\begin{array}{l}1.05 \\
1.05 \\
1.05\end{array}$ & $\begin{array}{l}\ldots 1 \\
\ldots 1 \\
\ldots 1\end{array}$ & $\begin{array}{l}\text { Sociometry } \\
\text { responds to more } \\
\text { powerful } \\
\text { intervention }\end{array}$ \\
\hline
\end{tabular}

Self-report $(.05 \quad$, ) More limited effect of ST

Roletaking $(.05,$,$) IT > ST$

$\begin{array}{lll}\text { Roletaking } & (.001 \quad,,) \\ \text { Self-report } & (.05 & \ldots, \\ \text { Sociometry } & (.05 & ,,\end{array}$

Self-report $(.05 \quad,$, 


\section{Discussion}

\section{Results, section 1}

In Table 1, the means suqgest that change took place in the predicted direction on the measures of sociometry and self-report. In other words, treatment produced some improvement as shown by each of these measures of social skill. This is shown by increase in score in the case of sociometry, reflectina raised social status, and by decrease in score in the case of self-report, indicating problem reduction. This finding holds good for both Individualised Traininq and Standardised Trainina. Clearly, further analysis is required to determine the level of significance of the changes and the relative dearee of improvement produced by the two types of training.

There is a suggestion that initially the subjects receiving Individualised Training had rather more social skills difficulty than the subjects receiving standardised Training. However this finding did not reach statistical significance. This tendency is in the right direction from the point of view of the research in that "the odds are stacked against the hypothesis"; however, the principle of "reqression towards the mean" is more likely to operate in the case of extreme scores.

When both groups are collapsed, the improvement in terms of the self-report measure is significant. It will be argued below that the self-report measure is more sensitive than sociometry. 
The Manova findings shows that when both groups are collapsed and the two measures are pooled, the overall improvement is significant. The Ancova results suggested that there was no difference in outcome between overassertive (possibly "rejected") clients and under-assertive (possibly "neglected") clients. This neqative finding is of interest as it suggests that both types of client may be helved by this form of intervention.

The Family Problem Test showed that subjects receiving Individualised Training initially had more family difficulties than the subjects receiving Standardised Trainina, although the finding did not reach statistical significance. As family difficulties may be correlated with social skills difficulties, the possibility of a correlation between family problem score and self-report was explored. No significant correlation was found.

The Roletaking Test provided support for a major prediction of the investigation, namely that subjects receiving Individualised Training would improve significantly more than subjects receiving the standardised Training. There was a siqnificant difference between the two forms of training in terms of their effect on the clients' change scores. This finding was further supported by the significant differences found on the clinical change score in Year 1 . Here again Individualised Training appeared to be superior to standardised Trainina. Other findings were consistent with the superiority of Individualised Training. Significant improvement in sociometric status occurred only in the pooled Individualised Training subjects. Therapist pair $B$ had siqnificant results in terms of sociometric change for Individualised Training subjects only. 
Overall, Individualised Training subjects showed more chanqe on more measures than Standardised Training subjects.

Improvement in sociometric status did not reach significance when all subjects were pooled. A possible explanation might be that sociometric chanqe is likely to occur over a longer time span than cognitive change or change in perceived social difficulty.

In addition to chanqe in sociometry and self-report, there was evidence of significant social-coanitive improvement as measured by the social Assertiveness Test and sianificant behavioural improvement as indicated by the Behavioural Interview Schedule. Change on the Role-taking Test (also a social-coanitive measure) is analysed in more detail in Results, section Two. Thus there is evidence that improvement may be produced on a socialcoqnitive measure without explicitly including a cognitive component in the treatment package.

It was unfortunate that owing to industrial action in the school it was not possible to obtain more detailed measurements of control group behaviour change nor was it possible to implement more than a few follow-up measures. Consequently it was not possible to obtain definitive evidence of the effectiveness of the intervention in statistical terms. Nevertheless the findings are consistent with the hypothesis that the intervention was effective. Using sociometry, no significant change was found in a "no treatment" control group from pretest to posttest. This makes it less likely that treatment chanqes may be accounted for by regression towards the mean. There was also no change on the staff questionnaire in the second 
year, whereas the treatment group run by Therapist pair A improved significantly on this measure.

The single case designs provide further details which aqain suggest that at least moderate improvement occurred in each of four randomly selected subjects receivina Individualised Training.

The Role-taking Test produced evidence of significant difference between the two pairs of therapist in terms of their effect on change scores. This dovetails with the therapists own iudqement of change as shown by the Clinical Chanae Ratina. Possible sources of difference between therapists are discussed in the final chapter. Differences between therapists are analysed in more detail in Results Section Two (Discussion).

Conclusion

There was evidence of significant improvement across a wide range of measures - behavioural, social-cognitive, self-report, and sociometric. Thus, the findings of study 1 were supported, in that there was further evidence of the effectiveness of a group training package with young adolescents in a school setting.

It appeared that the sociometric form of measurement was again somewhat less sensitive, even though a roster-rating system had been introduced. No doubt this was due partly to the time lag which is characteristic of this form of effect. The exclusion of neqative ratings of peers, on grounds of possible stigmatisation, may have further reduced the effect. Again, the measure may be prone to effects by extraneous (i.e. non-behavioural) 
variables, e.q. physical attractiveness, intellectual ablility, and being new to the neiqhbourhood.

There was reasonably strona though not definitive support for the prediction that the subjects receiving Individualised Training would do better than those receiving standardised Training. Overall, the Individualised Trainina subjects did perform better on a range of measures. Their Clinical Change Ratina was significantly better and their change score for role-takina (a social-cognitive measure) was also significantly superior.

A sianificant difference between therapist pairs in terms of effectivennes was demonstrated on the roletaking measure. Consequently, a further exploration of this variable was planned in Results, Section 2 . 


\section{Discussion}

Results, Section 2

A major difference in Results section 2 was the introduction of the Roletaking Test as a main outcome measure. The Roletaking Test was initially planned as a subsidiary outcome measure and was thought to be rather subjective as it was administered by the therapists. However the test was desiqned in a similar manner to an intelligence test and the therapists were appropriately trained in its use. From the conceptual point of view it was now seen as rather important to look at the Roletaking Test as a "social-coanitive" complement to the other two main outcome measures. The sociometric measure is clearly related to social status, a fairly "long-term" outcome of social skill; the self-report measure is related to variables such as "confidence", perhaps a medium-term measure in that it must depend to some degree on the experience of success; the Roletaking Test taps coonitive skill, a "process variable" which should respond immediately to social skill training.

Table 1 indicates that when all subjects are pooled there is highly significant improvement in two out of three measures of social skill. In Table 2 there is evidence of significant chanqe on all three measures; the Individualised Training condition is here assessed on its own merits, and a siqnificant improvement is found on sociometry as well as on the other two measures. Table 3 confirms that the Standard Condition has some effect, but a less powerful one, on sociometric status; significant improvement is now shown on self-report alone. On Table 4 the role-taking test, now emerging as the most sensitive measure, again provides clear evidence in support of the superiority of Individualised Training over 
Standardised Training. Surprisingly there was no sianificant difference between therapists in chanqe score on this occasion. However, Tables 6 and 7 demonstrate a difference of performance, confirming the previous finding in favour of Pair A. Pair A produced sianificant chance on all three measures, while Pair B produced significant chanqe on only one measure.

Summary.

There was further substantial evidence for the effectiveness of the intervention in that hiqhly siqnificant change was shown on two out of three measures when the results of all subjects were analysed together. There were further indications of the superiority of Individualised training over standardised training; subiects receiving the former had significantly better change scores on the roletaking test, and Individualised Trainina produced sianificant chanqe on all three measures, while standardised Trainina produced chance on only one measure. The roletaking test emerged as a very sensitive measure in a number of analyses, strongly suggesting the salience of comitive mediatina factors in the process of chanqe. There were some further indications of the superior effectiveness of Therapist Pair A. 
SOCIAL SKILLS TRAINING WITH YOUNG ADOLESCENTS

\author{
Chapter Four \\ General Discussion and Conclusions
}

Section

1.

2 .

3.

4.

5.

6.
Title

Overview of findings.

Review of research hypotheses.

The relationship between measures and outcome: A process view.

Areas for development.

Implications of the research.

Summary of implications. 
Overview of Findings.

Study I demonstrated the effectiveness of a group social skills training programme run for 9 young adolescents within a school setting and within the school timetable. By the time of six-month follow-up there was significant improvement in the subjects who received training on three relevant measures - social competence, sociometric rating, and self report Ten control subjects who did not receive the training did not improve in sociometric status. The findings stronqly suqgested that the intervention was effective and that qeneralisation occurred across time and across situations.

It appeared from study I that attending carefully to individual requirements for remediation might be an important component contributing to the effectiveness of the intervention. Therefore, Study II was designed to test this hypothesis and also to replicate the findings of study $I$.

Study II provided qualified support both for the effectiveness of the intervention and for the hypothesised superiority of individualised training. This supportive evidence came both from a group comparison design and from sinqle case designs. It was found important to monitor and control for therapist effects. There was evidence for differences in therapist performance. The crossover design of the experiment meant that 
the validity of the experiment was not impaired.

Taken together these studies do provide a substantial body of evidence for the effectiveness of interventions of this sort and the appropriateness of individualised training. The failure to provide what is technically considered definjtive evidence may be due to a number of factors. These include high variances, small numbers, and chance factors of a historical nature such as industrial action. Successful elimination of these obstacles must remain a challenqe for future research, though some of the difficulty mav be endemic to research in natural or "field settings".

\section{Verification of research hypotheses}

In the preliminary discussion and review of the literature, research hypotheses were implicitly or explicitly qenerated in six main areas, specifically:

i) The aqe oroup salected for intervention

ii) The setting selected for intervention

iii) The nature and significance of the "treatment packace"

iv) The siqnificance of the type of social difficulty experienced

v) The significance of "therapist effects" 
A seventh area of broad interest is the deqree to which the findings provide verification or otherwise of the basic postulates of the Argyle model (1967) later modified by McFall (1982).

Each of these areas of research interest will be considered in turn.

The age aroup

The clients offered intervention were aged $12-13$ and developmentally were in the early stage of adolescence. Adolescence is commonly supposed to occur in the second decade of life and is associated with a number of developmental tasks, e.q biological growth, sexual maturation, resolution of identity crises, completion of education, preparation for employment, attainment of social independence - to name but a $f \in w$ (Montemayor et al.,1990). By the end of the second decade many individuals are employed, fully independent, married and have children of their own. A large number of life tasks are to be tackled during this period which is characterised by rapid arowth and chanqe.

It is established that deficits in social competence in childhood and adolescence may be correlated with a variety of social and psychological difficulties in later life (Van Hasselt et al., 1979).

It may therefore be supposed that an intervention in early adolescence might enhance an individual's capacity to respond adaptively to the challenge of the adolescent period. If the individual suffers from some 
social impairment, intervention at the staige of early adolescence may prevent further deterioration.

The literature review arqued that early adolescence was a suitable development stage for the provision of a comprehensive social skills treatment packaqe.

The literature review further concluded that the research findings on social skills training with adolescents were (with the exception of certain groups) relatively sparse.

From this standpoint it is important to note that there was evidence of positive change in each of these two studies involiing altogether some forty youna adolescents of mixed aender. Study one provided objective evidence of generalisation across time and across situations. This finding would imply change in social competence as well as chanqe in social skill. Study Two provided:

i) objective evidence of change in social skill

ii) subjective evidence of change in social competence, in that clinical enquiry demonstrated enhanced confidence and enhanced ability to apply social-coanitive techniques.

Therefore, the two studies taken toqether support the hypothesis that intervention usinq a comprehensive treatment packaqe may be relevant and helpful to young adolescents identified as lacking in social competence. 
The setting

In the initial discussion it was proposed that an educational setting i.e, a school - miaht be an appropriate environment for the assessment and remediation of deficits in social competence. Examples of social skills training in schools were given from the literature le.g, Verduyn et al.. 1990).

Both studies showed that youna people's social competence deficits might usefully be identified by staff, and subsequently remedied as a result of appropriate intervention in school. Study Two showed in addition that deficits in social competence could be identified by peers within a school context, replicating similar findings from the literature (Oden and Asher, 1977).

Both studies showed that teachers and peers could usefully be involved in the provision of a social skills training programme within a school context.

Both studies showed that social skills training for students with identified need could be effectively integrated within the school curriculum.

Both studies showed that generalisation could occur within a school context.

Both studies showed that it was possible to do relevant work within school on an area frequently identified as a potential source of psychological trauma - namely the victimisation of young people by teasing and bullying. 
The treatment packaqe

It was hypothesised that a comprehensive treatment packaqe would be helpful to yound adolescents appearing to require social skills training. It was further suggested that owing to the psychological salience of the peer group in adolescence, the involvement of the peer qroup in training would be beneficial. Thirdly, the need for attention to individual need was emphasised. This need was documented in the literature review (Section 11). Study Two set out to test this third hypothesis systematically by a contrasted aroups desian.

Both studies provided evidence for positive change associated with a comprehensive group treatment packace, in which social skills training was offered to groups of six to nine younqsters in the form of a ten-session course. These findinas were consistent with hypotheses 1 and 2 .

In addition, the clinical case reports indicated that different clients experienced different types of difficulty and thererore it was important to offer a comprehensive package offering different social skills. The therapy supervision notes point towards a similar conclusion. Much of the discussion in supervision centred around the need to devise suitable exercises for individuals. On the other hand, the stimulation provided by peer reinforcement and feedback was also well documented in the clinical notes. There is therefore broad general support for hypotheses 1 and 2 from the various sources of information available. 
Consequently, there was some empirical support for the notion that a comprehensive aroup treatment backaqe is helpful for this age. In view of the paucity of studies with this age aroup, this information has considerable utility. There is clearly a need for further research to test these hypotheses in more detail.

The third hypothesis was supported by a wide range of findings using a variety of analyses in Study Two . Subjects receiving Individualised Training made significantly greater gains than subjects receiving Standardised Training on the Role-takina Test (a social-cognitive measure). Across all measures the gains made by subjects receiving Individualised Training were substantially arearer than those made by subjects receiving Standardised Training. In addition, the clinical case reports demonstrated the importance of working on individual deficits.

Consequently it would appear to be established that attention to individual deficits is of some importance in social skills training. This finding may well prove to be relevant for other client groups (Marzillier and Winter, 1978). It would be extremely interesting to test this hypothesis with a variety of client groups and to explore the possibility of replication of the finding with children and adolescents.

The type of social difficulty experienced

It was reported in the literature review (e.g. Asher, 1983) that two broad categories of social skills difficulty appear to be commonly experienced by children and adolescents - namely social rejection (associated with overassertion) and social neglect (associated with underassertion). Little is known about tic differential response of these conditions to intervention, and there is no guidance from the literature as to whether 
clients suffering from these two contrastina conditions may protitably be treated together.

In the two studies socially reiected children were initially identified by teacher assessment, and social neqlect was determined both by teacher assessment and peer nomination. By a breakdown of the self-report questionnaire items into those assessina underassertiveness and those assessing antisocial behaviour. it was possible to make a further differentiation.

The analysis in Study Two showed that there was no measurable difference in outcome for the two groups either on the repeated measures analysis (pooling self-report and sociometric data) or on the Roletaking Test change scores. Both aroups appeared to benefit from the intervention in study one, and the clinical analyses in study Two suqqested that clients in each group derived benefit, but in different ways. The underassertive clients acquired confidence from positive reinforcerent and enhanced their behavioural repertoire, while the overassertive clients built up their social-coanitive strategies and their self-control by applying sucaestions made by the social skills trainers.

Consequently the two studies taken together demonstrate that it is possible to provide helpful intervention through group social skills training programmes in which socially rejected and socially neglected clients are mingled. It may be that such procedures enable the contrasted groups to model on each other, thereby enhancing their behavioural repertoire. 
Therapist effects

The literature review emphasised the need to analyse the effect of therapist variables on outcome. Some outcomes may be due to the nonspecific effects of therapy. A "placebo" desion may not adequately control for such effects (Wilkins, 1986).

In an educational setting therapists of varying levels of experience may become involved in social skills trainina. Luborsky et al. (1975) suggest that therapists' experience may be a factor determining success in therapy. Teachers may have less therapeutic expertise than clinicians resulting in less successful therapy.

There appears to be some difference in the outcomes for Therapist Pair A (clinicians) and Therapist Parr B (teachers). When group outcomes were analysed separately Therapist Pair A achieved significant change on two measures in Year one and three measures in Year Two, whilst Therapist Pair B achieved significant change on one measure in Year Two only. Pair A produced significantly areater change than Pair B on the Role-taking Test. In the second analysis (Results Section 2) Therapist Pair A produced significant results on Roletakina and Sociometry over the two years while Therapist Pair B did not.

\section{Difference between Therapists}

It is clear from the study that there were systematic differences between the therapists affecting the performance of subjects and the degree of change shown on outcome measures. Therapist Pair A can be considered more experienced and perhaps more skilled in that their professional background 
was in a clinical setting and one of them was a trained clinical psycholoqist. Therapist Pair B were practisina teachers. Clearly they were experienced in handling aroups of students and in transmitting information to them but the "teacher" role may have made some sort of barrier between them and the clients, who may have had some difficulty in confiding in them. As teachers they may have had more intense and immediate competina pressures on their time; they may also have been more affected by "historical" factors such as the teachers' strike. The teachers also may have had some kind of problem with self-efficacy, believing therapy to be an alien skill in which they were not sufficiently "versed". Alternatively, gender factors may have been involved, in that Therapist Pair A were a mixed pair, while Pair B were both male; consequently female subjects trained by Pair A would have had a better role model than female subjects trained by Pair B. However, subject's gender did not appear to affect outcome on the Anova analy'sis (Study II, Results, Section 1). This finding casts doubt on gender as a key variable in therapist effects.

The outcome of this study is not affected by therapist difference since this is controlled for in the design. The study therefore establishes that there are effects due to the training package unconnected with therapist effects. It is clear that in other studies, for example those which seek to replicate an effect with a particular form of treatment, the therapist variable may need to be taken into account. You cannot just "plug in" a social skills packaqe and expect that the outcome will always be the same. The outcome depends partly on the therapist. 
Gender effects

The possible effect of therapist qender has been discussed. The analysis of covariance indicated that qender did not effect outcome on the pooled dependent measures of sociometry and self-report. The sinqle case desiqns suagested that clients of either aender were able to benefit from the intervention. Consequently it appears appropriate to employ a qroup social skills training package with young adolescents of mixed qender. The finding is useful since a number of comparable studies in the literature involved one gender only. The finding confirms intuitive expectations since gender issues are important for adolescents and therefore a positive experience in a small cohesive mixed group is likely to be beneficial.

The social skills model

The findings are in conformity with predictions that miaht be derived from the Argyle/McFall model for social skills training. There is evidence that cognitive and behavioural chanqe produced by social skills training leads to chanqe in social competence as observed by peers and adults.

To elaborate somewhat, the findings are consistent with the prediction that a "trouble-shootinq" approach to individual difficulties in social cognition and performance, providina detailed trainina on tasks as close to real life as possible, will lead to change in social competence as assessed by appropriate peers and adults usina appropriate criteria.

Therefore, the results tend to confirm the usefulness of the Argyle/McFall model. 
The Relationship Between Measures and Outcome: A Process View

The results indicate that in terms of the present study the measures are uncorrelated or "orthoconal". This may seem surprising in that the research assumes the existence of a measurable entity, "social skill" which the interventions seek to change and develop in certain ways. However, Hops (1983) draws a distinction between social competence and social skills. On this construction. social competence is assessed by the pooled judqement of social observers, while social skills are the specific behaviours of individuals which determine their social status. It may be that social skills are "situation specific" and therefore considerable analysis of natural situations is required before these behavioural attributes can be measured with any real accuracy. It is then not too surprising if the various measures of social competence and skill are found to be uncorrelated or loosely correlated.

Ladd and Asher (1985) identify three areas of functioning, each of which should be taken into account in an assessment of an individual's social competence and social skills. Firstly there is sociometric status; children low on this variable may be either "neglected" (having few friends) or "rejected" (unpopular owina to their social habits). Neglected children receive fewer positive attributions, rejected children get more negatives. Second, there are the behavioural antecedents of sociometric status. These may be assessed by observation of children in existing groups (Goldman, Corsini and DeUrioste, 1980) or by studyina children's behaviour in newly formed qroups (Coie and Kupersmidt, 1983). Thirdly there are underlying processes which may be coqnitive or motivational in nature. Gottman et al. (1975) found that low status children were less knowledqeable about how to make a friend than more popular peers. 
Children may act aquressively as a result of a faulty attribution of aggressive motivation to others; hence the source of the problem is coqnitive (Dodqe, 1980). Low sociometric status may be correlated with low self-efficacy (Wheeler and Ladd, 1982) and a sense of personal incompetence (Goetz and Dweck. 1980) : in such cases again the suqgestion is that the social difficulty arises from internal processes, though intuitively it may be supposed that there would be an interaction between the low status and (i) the sense of incompetence or (ii) low self-efficacy. To recapitulate, it is postulated that there may be three main relevant areas of social functioning: sociometric status, behavioural antecedents, and underlying processes.

It is consistent with the views of Ladd and Asher (1985) to suggest a model of change in which social skills training initially has an effect on cognitive/motivational brocess variables. This leads to the enacting of increasinaly successful performance of social behaviours reinforced by feedback processes along the lines of the Argyle model. As new habits are established the perception of the individual by the peer group and other social observers alters and there is a change in sociometric status. This model would be consistent with the findings of the present study in which the roletakina test shows the highest degree of change while the lowest change is on sociometric status. Clearly longitudinal studies are required to provide verification of this model. 
Areas for Development

In future studies it would be advisable to focus on process variables, both coqnitive and motivational. The term "process variables" (Ladd and Asher, 1985 ) is used to denote processes within the individual which lead to social skills difficulty. Process variables are to be distinquished from behaviours and sociometric status (Ladd and Asher, 1985). Process variables appear to be involved in some way both in the aenesis of social skills difficulty (Goetz and Dweck, 1980), (Wheeler and Ladd. 1982) and in the remediation of social skills (Curran, 1984), although the exact nature of the involvement is by no means clear. A process measure used in the current research - the role-taking test - provided evidence of significant gains from training. Further development of process variables would be helpful. It may be useful to develop new forms of assessment of process variables.

Conning and Head (1990) report that Callias and Likierman (Unpublished Manuscript) have developed the Social Cognition and Concepts of Friendship Test for use with younqer children. This is based on notions of social problem-solving (Spivack and Shure, 1980) and friendship making (Youniss, 1980). Measures of self-esteem and self-efficacy are also important, e.q The Children's Self-efficacy for Peer Interaction Scale (Wheeler and Ladd, 1982). These "social-coanitive" measures are mainly desianed for younger children, and would require sliqht adaptation for use with young adolescents. 
Implications of the Research

A main hypothesis of study II was that "tailoring" the input to individual needs would have a noticeable effect on treatment outcome. The results are consistent with such a hyoothesis but not conclusive. One implication is that it is worthwhile to include individual tailoring in social skills packaqes for youna adolescents, particularly in the stage of "training for qeneralisation", ie when assiqning tasks for real life practice. This findina is particularly relevant in cases where unassertive and antisocial subjects are mixed in aroup training as there will then be a greater ranqe of individual differences within the group. With more "one-dimensional" aroubs of subjects (ea Bornstein et al. 1977) individualised tailorina may not be so essential. Nevertheless, Hops (1983) arques for "a combination of current procedures that would provide specific interventions dependent on the specific skill deficits of each child".

The use of "qroup work" with young adolescents has been advocated in a number of studies (Bowdler and Gleisner, 1982; Verduyn $\in t$ al, 1990). The present investigation provided further evidence for the value of this type of intervention. The group was a powerful vehicle of learning for these clients. Therapist effects are clearly of importance in running social skills training groups with young adolescents; it is likely that this is true with other aqe groups and this area merits exploration in further research. 
Role-play (Sarason and Sarason, 1981) was used as a major component of treatment in both experiments. In providina specific interventions dependent on specific skill deficits role-play together with assignment settina and verbal rehearsal of the concepts involved must form a powerful ingredient of treatment.

other studies (Pellegrini and Urbain, 1985: Verduyn et al., 1990: Bowdler and Gleisner, 1982 ) have affirmed the value of school-based social skills treatment. The present research provides further validation for that tradition. The facilitation of sociometric techniques was found to be of particular benefit.

Summary of implications

The results reinforce the prodosition that a social skills packaqe containing a mix of current procedures, including verbal instructions, role-play, feedback and assignment of tasks qeared to individual deficits will be helpful to youna adolescents. Such a package may be delivered in the form of a group training proqramme within a school context. The training and monitoring of therapists is important. The findings are consistent with the view that outcome depends partly on selecting an appropriate intervention, partly on adapting intervention to the needs of the individual, and partly on therabist effects. Further research is required to explore these matters in more detail. 
SOCIAL SKILLS TRAINING WITH YOUNG ADOLESCENTS

Chapter Five

Appendices 
SOCIAL SKILLS TRAINING WITH YOUNG ADOLESCENTS

Chapter Five

Appendices

Table of Contents

1.

Course Manual

2 .

Single Case Designs

3.

The Family Questionnaire

4.

The Clinical Test Battery

5.

The Social Competence Questionnaire

6.

Therapist Self Rating

7.

Clinical Change Rating Scale

8.

Self Report Questionnaire (Short Form)

9.

10.

11.

Course Plan

Results for Sociometry and Self-Report

Measures: Breakdown by Group and Year

Social Skills with Young Adolescents:

published paper. 
Appendix 1

Course Manual

(Instructions underlined are issued to therapists for Individualised Training Groups only. For Sessions Seven to Ten, there are separate instructions for the two training conditions.)

Aims of Course

1. By creating an atmosphere of trust the therapists will provide an experience of positive social interaction within a structured setting.

2. Procedures for dealing with everyday social situations will be studied, developed and practised using modelling, role-play and. feedback. The therapists will control the feedback process but will bear in mind that direct feedback is a powerful reinforcer.

3. The training procedures will encourage clients to shift the focus of concern from self to others, attending more to others' feelings than their own, though appropriate assertion of their own rights and feelings will form part of the training.

4. Clients will learn to structure situations to enable them to practise newly acquired social skills and consolidate those that are already part of their repertoire. 
5. Clients will learn to be more aware of their anxieties in social situations, $\in q$ when authority figures or a degree of conflict is involved.

6. Clients will learn to generalise the skills learned and perfected in the group to other situations. "Special assignments".will be set to enable .. practice in "rẹl life". situations to occur. These special assignments. will be geared to the specific needs of individuals.

7. The therapists will be provided with social skills assessment profiles on each of the youngsters and will make conscious efforts to provide appropriate training to individuals in accordance. with their special needs, so long as the constraints of working in a group context are taken into account.

Format of sessions

1. Getting the group together

2. Warm-up (loosening up activity)

3. Main work (discussion / roleplay / homework setting)

4. Closure (eg favourite game) 


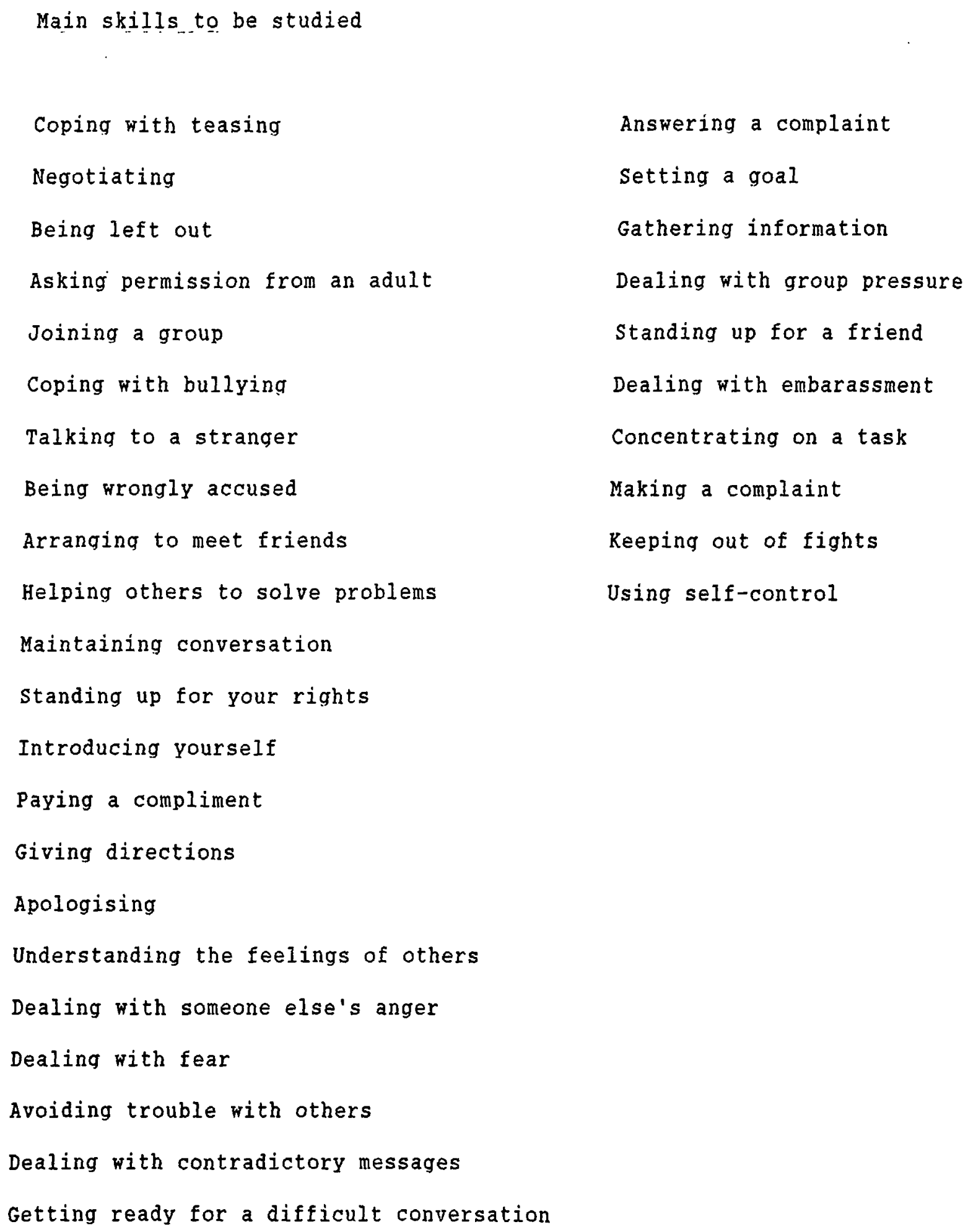

Answering a complaint

setting a goal

Gathering information

Dealing with group pressure

standing up for a friend

Dealing with embarassment

Concentrating on a task

Making a complaint

Keeping out of fights

Using self-control 
Games and Warm-ups

$\begin{array}{ll}\text { Name Game } & \text { Echo circle } \\ \text { Pass the cat } & \text { Blah-gab } \\ \text { Luckily/unluckily } & \text { Reflecting } \\ \text { Eye-wink murder } & \text { Knots } \\ \text { Passing the message } & \text { Blind partners } \\ \text { Sculpture of emotion } & \text { In the manner of the word } \\ \text { Expression of emotion } & \text { Physical proximity } \\ \text { Looking meditation } & \text { Leaning out circle } \\ \text { Meditation } & \text { Zip boing } \\ \text { Saying no } & \text { Yes/no } \\ & \end{array}$

1. The Name Game

Group members sit in a circle. One member says his name. The next member repeats that person's name and then says his own. This continues around the circle so that the seventh person has to say the names of the six previous people. When the circle is completed, start in a new place and go the other way round, but this time each person puts a descriptive adjective in front of his name and repeats the other names and adjectives, eg "Lazy Joe, Tough Tom, Quiet Joanne". 
2. Pass the cat

The group sit on the floor in a circle. Someone mimes holding a cat and then passes the imaginary cat to the person on one side of him, and so on round the circle. Continue with other objects, eg chewing gum, snake, length of elastic, bucket of slime.

\section{True Story}

Split group into pairs. A describes a true incident from his experience. $B$ repeats this with A's inflection and emphasis. A then tells the story again but in a different role - eg an absent minded old woman. Encourage partners to discuss the task afterwards and to give each other positive feedback.

4. Set the scene

Briefly outline the aims of the group and what you expect of group members. Deal with any questions that come up. 
Aims of session

1. Introduce group members to each other

2. Create cohesiveness

3. Beginnings of social interaction within the group

4. Introduce mime to draw attention to hand movements

5. Provide enjoyable experience

6. Engender motivation

7. Promote self-disclosure

8. Promote lively verbal exchange

9. Establish relationship with group

10. Set boundaries

Session. Two

1. The Name Game

See session one or try a different version; a member of the group throws a soft ball to another member, calling that person's name; that person throws it to someone else; and so on.

2. Looking Meditation

Split group into pairs. Partners should feel comfortable with each other. Partners should sit facing each other and after a while engage eye contact, and hold it. Say "Look calmly at your partner allowing yourself to become quiet and peaceful. If thoughts enter your head be aware of them but do not pursue them. If it feels uncomfortable, turn away and try again when you are relaxed." 


\section{Expression and Recognition of Emotion}

Get group to give you a list of emotion words that are meaningful for them, eg pain, pleasure, fear, amusement, surprise. Therapists model some of the words in a sculpt or "frozen" posture, using both face and body cues. All group members "mirror" the posture and facial expression - and then try to guess the word. Group members initiate new sculpts and the same procedure is followed for each. Draw attention to the various cues that are used to convey the emotion - facial, gesture, and posture. Give positive feedback for clear, direct cues and suggest improvements where appropriate.

\section{Friend across the street}

By this time leaders will have identified at least one lively pair which will be prepared to volunteer for a simple roleplay. Therapists should model it first. "You see a schoolfriend / aunty / teacher on the other side of a quiet street. Although you are busy you know you must go and greet them, otherwise they will take offence". Go through in advance the main stages of a common greeting:
a) Eye contact at a distance
b) Acknowledge eye contact with some gesture (eg raise eyebrows)
c) Brief verbal greeting ("Hi")
d) Approach without eye contact
e) Re-establish eye contact with friendly facial expression
f) Conventional question and answer reciprocated ("How are you" etc)
g) Take up conversational posture, start conversation linking greater with the other's world 
Assign each stage to a qroup member for observation - get them to report afterwards on whether it happened. Encourage direct and positive feedback.

5. Luckily / unluckily

Form circle. One begins, for example, "Luckily I was in time for school today. The next continues "But unluckily I got told off for not doing my homework"; and the next "But luckily she soon forgot about it because the headmaster came in". Go on round the circle; each statement must follow logically on from the last.

Aims of session

1. Build up group cohesiveness and sense of familiarity among members

2. Introduce vocabulary of emotions

3. Begin to emphasise importance of non-verbal communication

4. Introduce roleplay

5. Introduce notion of analysing a social interaction into its components 


\section{Introductory Exercise}

Use a variant of the Name Game, or "Our Day" in which each member has one minute to describe events of the day. In our day give positive feedback for genuine, appropriate expression of feeling.

\section{Sound Circle Echo}

Group sits in a circle. A word is chosen such as "Hello". "Please". "Mum". one member starts using a particular volume, tone, or pitch. The next member tries to repeat the word using the same intonation. The group then try to guess the mood that he was trying to convey.

\section{Sound Circle}

This time the intonation is varied by the next member, and the intended mood of both utterances is guessed.

\section{Questioning Numbers}

Now numbers are used as "pretend" words in a similar way. "A" puts a question with one number, using an interrogative tone; "B" replies with another number in a different tone (angry, fearful) and "B"s mood is guessed. Thus, further training is given round the group in the expression and recognition of emotion, using a new modality. 


\section{Eye contact_ Greeting}

The group sits in a fairly spread out circle. Individuals make eye contact with someone across the group and both acknowledge this by raising an eyebrow, or nodding if they can't do that. Continue for some minutes. Discuss how pleasant it is to have one's greeting acknowledged.

6. Physical Proximity - Roleplay

Therapist briefly model positions for conversation, confrontation, cooperation. Group observes, then practises in pairs. Positive feedback given.

\section{Eye Contact Murder.}

The murderer, selected by drawing a secret card, must select a victim and wink; the victim then "dies". The group must then detect the murderer.

Aims of Session:

1. Increase sensitivity to tone of voice

2. Increase ability to encode emotion by tone of voice

3. Develop vocabulary for this form of expression

4. Increase awareness of eye contact

5. Increase awareness of physical proximity

6. Enhance cohesion

7. Build confidence in role play and similar exercises, maintaining rapid tempo 
Session Four

1. Leaning out Circle

The group hold hands, and lean backwards. Thus, each individual is supportea by the group (therapists participate).

\section{BIah Gab}

A nonsense word exchalso game. Split into pairs. A starts off with 4 Blahs; $B$ repeats one Blah and adds a work of his own eg Gab, repeated 3 times, eq "Blah Gab Gab Gab" whereupon A responds "Gab Rhubarb Phubarb" and so on. Or continue with just Blah and Gab.

\section{Verbal Mirrorịng}

A relates experience, pausinq after each sentence; B repeats last part of each sentence, then asking question. A continues. Therapists model, group then practise in pairs.

\section{Verbal Reflecting}

As with mirroring; this time, B shows appreciation of how A must have felt after each sentence. 
5. Game

To be selected by aroup or theradist, from repertoire.

Aims of session

1. Training in active listenıng, facilitating, empathising

2. Promote skill and fluency in verbai self-expression

3. Further roleplay experience

4. Encourage mutual reinforcement 


\section{Session Five}

\section{Introductory Activity}

Therapists' choose appropriate activity.

2. Saying No

Go round circle. A asks B a favour: B must refuse. B must not apologise, but show recognition of A's need.

3. Roleplays

Joining a group

An outsider has to join an established group. Group brainstorms possible real life school/leisure situations; therapists have backup idea in case of need.

The roleplays should be planned by the therapist to develop both the "molar" and "molecular" skills of the individual based on prior analysis of his need.

Standing up for your rights

An aggrieved individual takes a defective article back to a shop.

4. Game 
Aims of session

1. Training in assertion

2. Training in more complex skills of social interaction

3. Observe clients' micro-skills "in action" and provide feedback

4. Begin to focus on "real life"

5. Encouraqe cooperation within group in working on "real life" skills

Note: by this stage, when roleplay skills are established, therapists should seize on any opportunity for useful role play presented by the reported experience of individual group members, and avoid rigid adherence to the session plan. 
1. Our Day.

2. Yes/No - in pairs. A says Yes, B says No, and so it goes on; then change over. The exchange acquires momentum as the participants try different intonation strategies and respond reciprocally.

3. Roleplays

Dealing with bullying

Brainstorm to elicit a "real life" peer situation. Aqree on roles and establish a verbal "prompt" to set the roleplay in motion.

Being wrongly accused

A confrontation, probably with an adult. Roleplayer must steer middde course between resentful reaction and overcompliance. Discuss alternative strategies. Group to give positive feedback to roleplayer.

4. Game 
Aims of session
1. Continue work on assertion
2. More fine-grained analysis of interaction
3. Maintain high level of group rapport
4. Encourage feedback from group to individual

Session Seven

Individualised Treatment Groups

1. Our Day

2. Eye Contact Greeting

3. Special Assignments - Introduction of Concept

Introduce notion of individual "Special Assignments" over the next three weeks. Allocate individuals to sessions. Explain purpose of assignments, ie practice in real life situations.

\section{Roleplays}

Select themes geared to needs of individuals undertaking assigments this week, eg negotiating, paying a compliment, understanding the feelings of others. Highlight points that will be useful to those doing assignments. 


\section{Special Assignments - Setting Tasks}

Discuss tasks, which should be tailored to remediation of the deficits of the individual concerned. Tasks should be practicable and neither too easy nor too difficult. Opportunities for practice (ie carrying out the tasks in real life) should be plentiful. Put details of agreed task on white card as reminder.

6. Game

Aims of session

1. Introduce concept of Special Assignments

2. Assign real-life tasks appropriate to individual need

3. Development of relevant skills through roleplay

4. Promote qeneralisation 


\title{
Standardised Treatment Groups
}

\section{Our Day}

2. Warm Up

Therapists select suitable activity.

3. Roleplays

\author{
Negotiating \\ Paying a compliment \\ Understanding the feelings of others \\ Keeping out of trouble
}

4. Homework Setting

Select a simple task associated with the roleplays, eg paying someone a compliment. Ask each member of the group to carry this out in real life and report back next time. The task should be neither too easy nor too difficult and each client should have plentiful opportunities for carrying it out. Give each client, by way of a reminder, a white card on which details of the task are noted. 
5. Eye Wink Murder

Aims of session

1. Continue to develop more complex skills through roleplay

2. Offer real life opportunities to practise the skills acquired

3. Promote generalisation 
1. Our Day

2. Warm Up

3. Special Assignment Feedback

Check on tasks and provide appropriate positive reinforcement. offer opportunities to try again in cases where tasks are not completed. Maintain expectation that the tasks will be carried out, as long as there are no insuperable obstacles. Discuss any problems encountered, employ problem solving approach. Involve group in discussion of problems presented by each task.

4. Roleplay

Therapists plan appropriate roleplay with 5 in mind.

5. Special Assignment Setting

Discuss and agree assignments for new individuals.

6. Game 
Session Nine

Individualised Treatment Groups

In Session Nine the same format is repeated, assigning appropriate tasks to the remaining members of the group.

Session Eight

Standardised Treatment Groups

1. Warm Up

2. Homework Assignment Feedback

Check on assignments and ask clients who did not succeed to have another go. Provide appropriate positive reinforcement.

3. Roleplays

Dealing with embarassment

Talking to a stranger

Offering help 


\section{Homework Setting}

As before assign a real life task, based on one of the role plays, for the whole group to carry out. Specify as much detail as possible.

\section{Game}

Aims of session

1. Promote conversational skills

2. Promote assertion

3. Help generalisation 
1. Questioning Numbers

2. Homework Feedback

Provide appropriate positive reinforcement.

3. Roleplay

Therapists to select appropriate roleplay.

4. Homework Setting

Involve group in active planning of tasks.

5. Game

Aims of session

1. Train in sensitivity to tone of voice

2. Maintain process of generalisation to real life

3. Continue to rehearse complex skills 


\section{Session Ten. \\ Individualised Treatment Groups}

1. Our Day

2. Future Plans

Discuss ending of group, and specify plans for further individual assigniments .

3. Special Asșignment Feedback

4. Training for Generalisation

Suggestions are made for maintaining skills in real life, with specific reference to individuals.

5. Roleplay

Repeat successful earlier roleplay with maximum group participation.

6. Video playback

Group views playback of roleplay on video. Therapists highlight newly acquired skills of individual members, as demonstrated on video.

7. Game 
Aims_of seșịion

1. Prepare group for disengagement

2. Highlight gains that have been made individually and collectively

3. Make positive use of group processes which may be intensified in this final session

4. Stress importance of generalisation 
1. Warm Up

2. Homework Feedback

Provide positive reinforcement.

3. Wind up Group

Summarise achievements of qroup. Emphasise need for continued practice.

4. Roleplay

Re-run of previous successful roleplay, perhaps with minor variation.

5. Video Playback

Group views playback of roleplay. Highlight improved standard of group as a whole.

6. Game 
Aims of session

1. Prepare group for disengagement

2. Highlight achievements of group

3. Promote generalisation

4. Positive reinforcement

5. A rewarding end to the course 
Appendix 2 .

Single Case Designs

The following case reports were prepared at the end of the Individualised Treatment Programme (Year 1) by the male member of Therapist Pair A. Each report is followed by an assessment profile, a qualitative interpretation of results on 3 psychometric instruments, and an evaluative comment.

i) John

Case report.

John had very mixed feelings about the group. His comment was "It's better than doing maths." He feels that he has made more friends since being in the group and thinks that the group helped him with this.

He said that he was referred to the group because "I am weird". He said that he needs to be weird because the friends he has expect it of him. When I asked him when he would be able to drop this weird bit of himself, his reply was "when I leave school". He talked about the possibility of his family moving to a different area, and said that if he moved to another school he might be able to drop the "weird bit".

John is the youngest of a family of five children but does not feel that he is treated like the baby of the family. Unlike June, I think he has to compete very hard in this large family to get his needs met. 
John talked sensibly and appeared to have a good understanding of his difficulties and how they might be detrimental to his future.

He told me that he had a fight this morning. We talked about why this happened in some detail. He said that his cat died and he is quite sure that it had been poisoned by someone. He felt both angry and upset about this and really did not know how to handle it. He did know that the boy whom he attacked had not in fact harmed his cat; he had just teased him a bit that morning. He was able to see that he had unleashed his angry feelings about the harm done to his cat upon this boy.

John seems to understand that he loses control and expresses his anger towards the wrong people for the wrong reasons.

I asked John if he would like a further group experience of a similar kind. He said that he would definitely come back to the same group with the same group leaders, but that he would not want to start again with another group.

John clearly does need ongoing help and it might be appropriate to offer him the booster group to see if he will accept it.

Prior to seeing John I had a brief discussion with the Headmaster, who informed me that he had referred John to the Educational Psychologists because of his outbursts. He felt that the school had exhausted all the various approaches with him. 
As John had not been told about this I did not discuss it with him. There is clearly a need for further work and it might be appropriate for the Educational Psychologists to look at what kind of programme might be set up within the school to help him.

John: Așsessment profile.

\begin{tabular}{|c|c|c|c|}
\hline Measure & Pre & Post & Direction \\
\hline Sociometry & 0 & 3 & Improvement \\
\hline Teacher nomination & 1 & $1 / 2$ & Improvement \\
\hline$S \in l f$ report & 19 & 27 & Deterioration \\
\hline Social assertiveness & 10 & 8 & . \\
\hline Role-taking & 8 & 8 & No change \\
\hline Behavioural interview & 3 & 6 & Improvement \\
\hline Staff questionnaire & 32 & - & \\
\hline $\begin{array}{l}\text { Bristol social } \\
\text { adjustment guide }\end{array}$ & 16 & - & \\
\hline $\begin{array}{l}\text { Family } \\
\text { questionnaire }\end{array}$ & 17 & - & \\
\hline
\end{tabular}

Self report questionnaire.

John underlined the item : (Do you) "Find it hard to keep your temper when an adult won't let you do something". He ringed 6 "antisocial" items and 13 "unassertive" items. 
Family questionnaire.

John came from a "traditional" two parent family. The family were quite aware of his problems in making and maintaining friendships and in controlling his behaviour. There were frequent arguments at home with all family members. It was felt that he had difficulty relating appropriately to staff and was teased or bullied by peers at times. He was judged to have severe difficulty with eye contact and moderate difficulty with facial expression, fidgeting and fluency of speech.

\section{Clininal change rating.}

On the Clinical Change Rating, John was felt to have improved in expression of feeling, expression of opinion, positive statements about others, praising others, disagreeing with others, and conversation skills. He was felt to be unchanged in respect of avoiding disputes, refusing unreasonable requests, positive statements about self, and accepting compliments.

Evaluative comment.

Prior to treatment, John was perceived by staff as a rejected, antisocial child (this is the significance of the teacher rating). From the staff questionnaire, it is clear that the staff believed that he had a good deal of difficulty in social interaction. The Bristol Social Adjustment Guide indicated that he had a problem of overreaction. The Family Questionnaire indicated that he had a good deal of difficulty both in terms of problem severity and "micro-skills". He was clearly quite isolated from the peer group. 
Post treatment he showed a very slight improvement in sociometric status. The teacher rating chanqed from 1 (antisocial) to $1 / 2$ (antisocial/unassertive). This would suggest he might have been overreacting less. The abparent deterioration in self report may have been due to a more accurate appraisal of his problems. There is evidence from the Behavioural Interview Schedule that he was perhaps able to control his behaviour rather better, though the gloomy prognosis of the Head would appear to cast some doubt on this point. John's comments at interview and the Clinical change Rating do indicate that at least moderate improvement occurred, even though this was clearly not sufficient to keep him out of trouble.

Consequently the psychometric indicators in conjunction with the clinical interview strongly suggest that a clinical improvement occurred which statistical analysis might not have detected. This was an extremely "problematic" client, and a "miracle" cure is not claimed, but it is valuable to know that the treatment package had some relevance to his needs. 
ii) June

Case report.

June said that she enjoyed the group and would have wanted it to continue. The reason she enjoyed it, she said, was because she never felt left out as she had felt in other aroups.

She said that she did not understand why she had been recommended for the group and then went on on to tell me why - "Because of my isolation"!

She said that thinas were much better for her now, and that she had more friends than she did previously. In dancing classes at the school, she had always been the one left out, without a partner. Now she was able to make sure that she got a partner, even if it was a boy. "I just go and grab one now".

She is the youngest child and the only girl in a family of five children. She said that her mother was asthmatic and suffered from migraine attacks. The children share the housework. June suffers from eczema and expects to suffer from asthma when she is older.

June is actually quite socially skilled. As the youngest and only girl in a large family it is likely that she has not needed to fight for attention or to assert herself much to get what she wants. She is quite capable of doing this if she needs to.

I do not feel there is a need for a booster group experience. June should do quite well enough without this. 
June: Assessment profile.

\begin{tabular}{lccc} 
Measure & Pre & Post & Direction \\
\hline $\begin{array}{l}\text { Sociometry } \\
\text { Teacher nomination }\end{array}$ & 2 & 3 & Improvement \\
$\begin{array}{l}\text { Self report } \\
\text { Social }\end{array}$ & 33 & 26 & Unassertive \\
$\begin{array}{l}\text { assertiveness } \\
\text { Role-taking }\end{array}$ & 8 & 8 & Improvement \\
$\begin{array}{l}\text { Behavioural } \\
\text { interview }\end{array}$ & 7 & 8 & Improvement \\
$\begin{array}{l}\text { Family } \\
\text { questionnaire }\end{array}$ & 3 & 4 & Improvement \\
\hline
\end{tabular}

Self report questionnaire.

On the self-report questionnaire June had 28 "unassertive" items and 5 "antisocial" items. She underlined (Do you)"Find you don't have many friends" and "Wish more people liked you".

Family questionnaire.

The family was a traditional two-parent type. The family were in agreement with the assessment of school that June was socially isolated and unresponsive. There were frequent disputes at home, mainly with her siblings. She was not withdrawn at home. She was not thought to relate appropriately to staff. She was thought to be isolated and avoid interaction at school. She had difficulty in relating to peers outside school. She had difficulties with her grandmother. No difficulty with "micro-skills" was reported; in fact these were described as quite good. 
Clinical change rating.

on the Clinical Change Rating June was thought to have made substantial gains. With regard to self-expressive skills she had done particularly well in expressinq her opinion, and had also made gains in expressinq feeling, accepting compliments, and making positive statements about herself. Her other-enhancina skills had also improved, in particular making positive statements about others, praising others, and disagreeing with others. Her communication skills were also better, especially her conversation skills.

Evaluative comment.

Initially June was assessed by teachers as unassertive. Her sociometric status suggested that she was isolated. In interview she admitted she had been "left out". The family assessment suqgested the presence of moderate social difficulty.

On completion of treatment, June had made modest qains in sociometric status, and improved considerably in social interaction both by her own statement and as shown on the self-report measure. She showed improvement on the Role taking test (a social-cognitive measure) and on the Behavioural Interview schedule. The therapist considered that substantial gains had been made. It was aqreed that no further input was required. There can be 
little doubt that this was an appropriate and quite successful intervention.

iii) $\operatorname{Ian}$

Case report.

Ian said that he felt uncomfortable for the first few group sessions but then began to relax and enioy it.

He thinks that he was referred because he was too shy. He now feels less shy and is sure that the aroup helped him with this. He talked about being able to go into a shop now without feeling uneasy. He said that he would still like to be "even less shy", but clearly he has benefitted.

He does not know his natural father, who apparently left his mother when he was still a baby. His mother then remarried and his two younger brothers are of that union. Two years ago his mother and stepfather split up and his mother moved to the area at this time with her new boy friend. Ian likes his new stepfather and is hoping that his mother will marry this man shortly. Ian said that he would appreciate more Social skills input and I feel that he would benefit from some booster sessions. 
Ian: Assessment profile.

\begin{tabular}{|c|c|c|c|c|c|c|}
\hline Measure & $\mathrm{P}$ & & & Post & & Direction \\
\hline$\cdots \cdots \ldots$ & .. & - & $\cdots \cdots+$ & $\cdot \cdots \cdots$ & $\cdots+\cdots$ & $-\cdots+-m$ \\
\hline Sociometry & 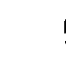 & & & 6 & & Improvement \\
\hline Teacher nomination & 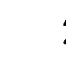 & & & 2 & & (Unassertive) \\
\hline Self report & 32 & & & 25 & & Improvement \\
\hline Social assertiveness & 11 & & & 7 & & Deterioration \\
\hline Roletaking & 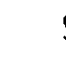 & & & 9 & & No change \\
\hline Behavioural interview & 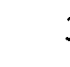 & & & 5 & & Improvement \\
\hline Staff questionnaire 2 & 20 & 4 & & & & \\
\hline $\begin{array}{l}\text { Bristol social } \\
\text { adjustment quide }\end{array}$ & 17 & 3 & & & & \\
\hline $\begin{array}{l}\text { Family } \\
\text { questionnaire }\end{array}$ & 5 & 7 & & & & \\
\hline$-\cdots+\cdots-\cdot$ & & - & & & $-\quad \cdots . .$. & $-\cdots$ \\
\hline
\end{tabular}

Ian ringed 25 "unassertive" items and 7 "antisocial" items. He underlined (Do you) "Find you lose your temper easily".

Family questionnaire.

The family was "reconstituted" as described by Ian. The family agrreed with the staff's assessment of Ian as socially isolated and unresponsive, having difficulty in initiating and maintaining contact with other children. Disputes occur with siblings and contact is avoided with family generally, though in aeneral he relates to the adults well. The main problem in school is that he is isolated and avoids contact with other 
children. He also has difficulty in relating to peers outside school. Severe difficulties are reported in eye contact, fiddling, and fluency of speech.

Clinical change rating.

On the Clinical Change Rating there were indications of gain. Selfexpressive skills had improved, in particular "Expression of opinion" and "Positive statements about self". Other-enhancing skills were also better, notably "Disagreeing with others". Communication skills had also shown benefit.

Evaluative comment.

Prior to assessment Ian was clearly a "problematic" young man. He was of low sociometric status, and assessed as unassertive by staff. The teacher questionnaire sugqested substantial difficulty at the level of microskills, whilst the Bristol Social-Adjustment Guide indicated underreaction. The family questionnaire gave evidence of very poor microskills, and isolation from peers.

Post treatment, Ian shows improvement across a range of measures. He has made modest gains in sociometric status and has a better view of his skills in interaction as evidenced by the self-report measure. He has improved on the behavioural interview schedule, though the social assertiveness score is disappointing. Gains are shown on the clinical Change Rating. subjective report in the clinical interview is likewise suggestive of modest improvement. It appears that that there may have been emotional and social disruption in the past with possible lack of an adequate male role model. There are indications that the situation is stabilising. 
As in the case of John, there is no "miracle cure" in the case of this young man who is clearly a cause of considerable concern, though he appears to be a "neqlected" rather than a "rejected" client and therefore the prognosis may be rather better. Certainly he needs booster sessions. There is however again strona evidence that he has been appropriately selected for intervention and has derived some benefit.

iv) Sharon

Case report.

Sharon felt that the Social Skills Group was a good experience for her. She felt accepted and comfortable with the group.

She told me that she was referred to the group because of her bad temper. She said that she can now keep her temper better and is making more friends.

Sharon has an older sister who, according to her, is very beautiful and wants to be a model. Sharon's remark about all this was "It really cheeses me off". She talked about not wanting to take a boy friend home because she knows they will prefer her older sister. 
It seems that in Sharon's family Dad is the authority figure. She has many arquments with Dad (as does her older sister) and feels that it is because of his attitude.

It appears that the girls are unable to arque with their mother because she is sick. Sharon is not sure what the problem is but she sometimes falls and bruises herself.

I gave Sharon a good deal of bositive feedback about her performance in the group. I told her how competent she could be if she chose. I suggested she go on working on not losing her temper and on making and keeping friends.

Sharon obviously made full use of the qroup. She was able to reiterate some of the helpful suqgestions made in the group that she has been able to apply. I do not think that Sharon needs a boostei group experience, and making due allowance for the fact that she wanted to please me, I think she has gained a good deal and will do well enough. 
Sharon:_Assessment prọile.

\begin{tabular}{|c|c|c|c|}
\hline Measure & Pre & Post & Direction \\
\hline Sociometry & 11 & 9 & Deterioration \\
\hline Teacher nomination & 1 & - & Antisocial \\
\hline Self report & 27 & 28 & Deterioration \\
\hline $\begin{array}{l}\text { Social } \\
\text { assertiveness }\end{array}$ & 6 & 5 & Deterioration \\
\hline Roletaking & 7 & 7 & No change \\
\hline $\begin{array}{l}\text { Behavioural } \\
\text { interview }\end{array}$ & 4 & 6 & Improvement \\
\hline Staff questionnaire & 50 & - & \\
\hline $\begin{array}{l}\text { Bristol social } \\
\text { adjustment Guide }\end{array}$ & 18 & & \\
\hline $\begin{array}{l}\text { Family } \\
\text { questionnaire }\end{array}$ & 18 & & \\
\hline
\end{tabular}

Self report questionnaire.

Sharon ringed 13 "unassertive" items and 14 "antisocial" items. She underlined (Do you) "Find you don't have many friends". She misinterpreted two of the pictures on the social skills test battery. 
Family questionnaire.

The family was of the traditional type. The family agreed with the view of staff that "Sharon was overassertive, creating tension and alienating others, eg dominating discussions, never listening to others, showing physical aggression, and beina short-tempered". Disputes were frequent and severe particularly with siblinas. Contact with family tended to be avoided. She was thought to have poor relationships with staff, and to have excessive disputes with other children. She was not able to make relationships with peers outside school. A severe difficulty with fidgeting was identified and moderate difficulties with eye contact and posture พอre reported.

clinical change rating.

The results of the clinical Change Rating were extremely positive. Sharon made gains in all areas with the exception of "Positive statements about self". She made substantial improvements in the following items: expression of feeling, expression of opinion, accepting compliments, positive statements about others, praising others, disagreeing with others, conversational skills, and avoiding disputes.

Evaluative comment.

Sharon's sociometric status was only moderately low prior to treatment. She was identified by the teachers as "antisocial". In terms of self report her difficulties were less severe than some other members of the group. However her initial score on the Behavioural Interview Schedule was quite low, suggesting that at times her behaviour became uncontrolled 1 and she 
was aware of it. A fairly high level of problem severity was reported by the family. The Bristol Social Adjustment Guide shows a tendency to overraction. The teacher questionnaire indicates that Sharon's deficits in social skills were only moderate. From the clinical interview, it would appear that there were a number of proximal antecedents related to her position in the family and her stace of development.

It would appear that whilst different from some other clients Sharon was appropriately referred in that she was perceived to have poor social adjustment and was aware of her difficulties in anger control.

At first sight, the psychometric information on outcome is not encouraging. However it should be borne in mind that sharon started from a relatively high baseline in terms of skill, and that her current problem appears to be one of retaining control of her emotions in relatively stressful circumstances. Consequently, the one point drops in sociometric status, social assertiveness and self report would seem to be of little significance. However the two point gain in her own prediction of behavioural effectiveness may have more importance. It is unfortunate that post treatment data on the Bristol Social Adjustment Guide are not available.

Sharon felt that she derived benefit from the group, and made more friends as a result of it. Further, the clinical change rating was extremely positive, and there was improvement on one social-cognitive measure. Sociometric change may laq behind changes in social interaction and Sharon's subjective report of improvement is therefore congruent with the psychometric data. Therefore, the therapist's assessment and Sharon's 
subjective report together with social-cognitive data sugqest that substantial gains may have occurred here which would not be detected statistically.

(There may, of course, be a clinical equivalent of the Hawthorne effect here, in that the therapist, a friendly male adult, may have been able to establish a very positive rapport partly because of the girl's difficulties with her own father; equally, it may be in cases where the difficulties are largely situational that the individualised approach is particularly helpful. We should then look for situational rather than psychometric change and it is unfortunate that more evidence of this is not available.) 
Appendix 3

The Family Questionnaire

1. Does $X$ have problems within the family?

2. Are they frequent?

3. Are they prolonged?

4. With Father? Mother? Other children?

5. Is contact avoided with Father? Mother? Other children?

6. Does $X$ have good relationships with teachers?

7. Is $X$ bullied in school?

8. Is $X$ involved in excessive arguments in school?

9. Does $X$ avoid contact with others in school?

General Social Behaviour

$\begin{array}{ccccccc}\text { Please rate } & 1 & 2 & 3 & 4 & 5 \\ & & & & \\ \text { (poor) } & & & & & \text { (qood) }\end{array}$

on each of the following items:

a) When in converation does X look at you?

b) How does $X$ respond to questions?

c) Posture

d) Use of tone and pitch in speech

e) Clarity of speech

f) Fluency of speech

g) Facial expressiveness

h) Laughs / smiles when appropriate

i) Use of gesture (hands) to indicate meaning

Alternative Form

See following sheet. 


\section{Appendix 4 \\ The Clinical Test Battery}

Materials

1. Set of 8 line drawings from Rosenzweig Picture Frustration Study, $17-24$.

2. Set of record forms for recordina responses to social assertiveness test, role-taking test, and behavioural interview schedule.

Instructions

Social Assertiveness Test

"I want you to tell me sensible ways that people in these pictures can stand up for their rights."

Indicate the child identification figure on the right, ask what should go in the "ballooon", and record the verbal response on the record form or audiotape.

Repeat procedure for each of the eight pictures in the sequence $17-24$. 
Role-taking Test

"What would the person on the left (girl, boy, lady) say then?"

Press for a reasonably elaborated response, or accept a short sharp one, eq "Get lost" ; but avoid leading or steering the child to any particular type of response, and do not attempt to get the child to improve the response. Use prompts like "Tell me a bit more" if a fuller response is needed.

Behavioural Interview Schedule

(For oral administration)

What do you usually do if:-

1. You are talking to someone you don't know well and you run out of things to say?

2. Are with some friends and find that you are doing most of the talkinq?

3. Want to go up and talk to someone but feel nervous about it?

4. Want to say you are sorry to an adult but don't know how to go about it?

5. Find other kids are trying to get you to do something that you know is wrong? 
Usinq neutral prompts, elicit at least a full sentence that can be recorded (either in writing or on audiotape). If the child says that it does not ever happen, ask "What if it did?" Try to qet the child to report an actual situation and describe what occurred.

If all attempts to elicit a suitable response fail. record "non-scoreable". If possible note suspected reason for non-response, eq test anxiety, problem with recall or imagination.

\section{Scoring}

Social Assertiveness Test

A response which assures the rights of the identification figure whilst recognising the needs of others, showing a high level of social skil1-

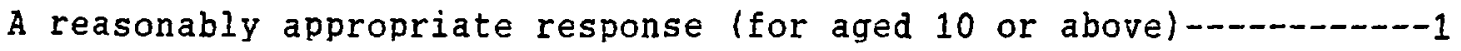

An innappropriate or immature response (eg not wanting parents to go out on $17-0$ 
Role-taking Test

A very good assessment of the probable response--

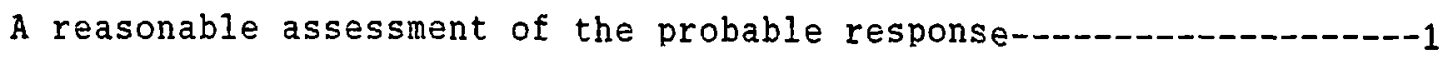

An inappropriate response-

Behavioural Interview Schedule

Hiahly skilled approach to the problem--

A reasonable approach for this aqe group-.....

An inappropriate or immature approach-_... 


\section{Appendix 5}

The Social Competence Questionnaire

Please rate the child from $A$ to $E$ on the following:
a) Intellectual potential
k) Responding to failure
b) Academic performance
1) Asking permission
c) Ability to mix with peers
m) Making a decision
d) Asking a question
n) Concentrating on a task
e) Keepinq conversation qoinq
o) Dealing with group pressure
f) Following instructions
g) Asking for help
h) Responding to teasing
i) Sharing
j) Avoiding trouble with others

Nature of problem

1. Where does the problem behaviour happen - in school or outside?

2. If in school, in what situation?

3. How often does it occur (ea how many times per week)?

4. Does it occur with peers?

5. Does it occur when the child is being disciplined?

6. How does the child cope with self control?

7. How does the child cope with being left out?

8. What do you think miaht be the reason for the problem behaviour?

9. What approaches have been used by staff in dealiong with the problem?

10. Any other comments?

Note

A problem severity score was derived from the second section using defined criteria, and this formed a component of the score. 


\section{Appendix 6 \\ Therapist Self Rating}

Show by ringing the appropriate number how you would rate yourself on the following dimensions:

a) Involvement in the group

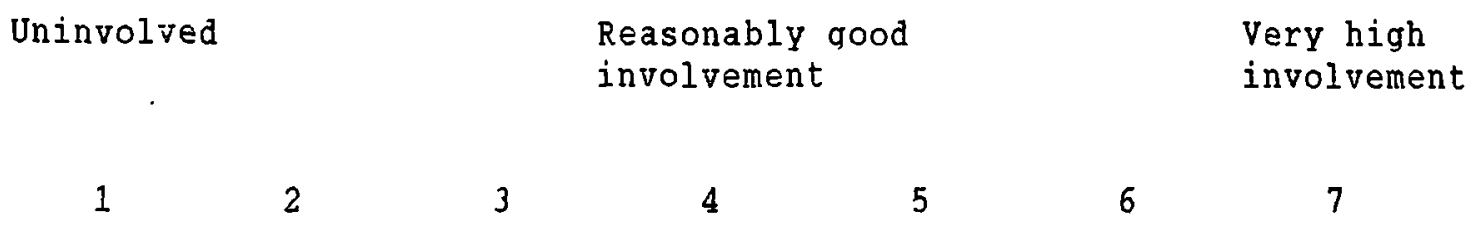

b) Proficiency in modelling social skills

(Format as above)

c) Grasp of concepts

(Format as above)

d) Management of programme

(Format as above)

e) Manaqement of roleplay

(Format as above)

f) Skill in giving positive feedback

(Format as above) 
g) Use of self-disclosure

(Format as above)

h) Ability to generate ideas

(Format as above)

i) Flexibility in planning

(Format as above)

j) Ability to maintain boundaries

(Format as above) 
Appendix 7

Clinical Change Rating Scale

Function

The Clinical Change Rating Scale was used to assess clinical change in Experiment 2 .

origin

The dimensions used were initially sugqested by Rinn and Markle (1979).

Instructions

Show how much you feel the client has changed, and in what direction, on the following dimensions, by ringing what seems to be the right number on each item.

1. Self-expressive skills

a) Expression of feeling

Much worse expression No change $\begin{aligned} & \text { Much better } \\ & \text { of feeling }\end{aligned}$
$\begin{array}{ll}\text { expresion of feeling }\end{array}$

12

3

4

5

6

7 
b) Expression of opinion

(Format as above)

c) Accepting compliments

(Format as above)

d) Positive statements about self

(Format as above)

2. Other-enhancing skills

a) Positive statements about other

(Format as above)

b) Praising others

(Format as above)

3. Assertive skills

a) Disagreeing with others

(Format as above) 
b) Refusing unreasonable requests.

(Format as above)

4. Communication skills

a) Conversation skills

(Format as above)

b) Avoiding disputes with peers

(Format as above) 


\section{Appendix 8}

Self Report Queștionnaire (Short Form)

1. Do you enjoy a good chat with people you like?

2. Do you feel shy with people you do not know?

3. Do you have many friends?

4. Do you wish that more people liked you?

5. Do you find it easy to talk to strangers at a disco?

6. Do you get teased a lot?

7. Do you lose your temper easily?

8. Do you find it easy to make new friends?

9. Do you enjoy helping others?

10. Do people often ask you for favours? 
Appendix 9

Course Plan

(study one)

Overview

Session No.

Function

Method

1.

Induction

Assessment

2.

3.

$4 / 5 / 6$.

$7 / 8 / 9$.

10.
Assessment

Observation

Assessment

Training̣

(based on

assessment of

individuals)

Individual

homework

Conclusion

Re-evaluation
Task rehearsal Feedback on task Reinforcement

Review progress

Role-play

Questionnaires 
Structure of Sessions

1.

Introduction

Name Game

Blind Partners

True story

Contract

2.

Name Game

Pass the Cat (Mime)

Role-Play (Coping with being Teased - Group A)*

,. (Negotiating - Group B)

Fortunately/Unfortunately (Group Story)

3.

Name Game

Eye Wink Murder

Passing the Message

Role-play (Being Left Out - Group A)

Role-play (Asking Permission - Group B)

Fortunately/Unfortunately

4.

Introduction

Discussion of goals

Eye Contact Exercise

Role-Play

Fortunately/Unfortunately 
5.

6.

7. 
8.

Expectations (Reminder of Outing)

Meditation

Feedback on Assiqnments

Reinforcement (Stars on chart and verbal praise)

Task Setting

Role Play (Task Rehearsal)

Feedback on Behaviour in Session

9.

Feedback on Final Assignments

Reinforcement

10.

Review of Individual Work

Reinforcement

Goal setting for Future

* Groups were split here and later according to individual need. 


\section{Appendix 10}

Results for Sociometry and Self-Report Measures: Breakdown by Group and Year

Year 1

Individualised condition.

\begin{tabular}{ccccc} 
Client & \multicolumn{2}{c}{ Sociometry } & \multicolumn{2}{c}{ Self-report } \\
& Pre & Post & Pre & Post \\
& $\ldots$ & & - & - \\
1 & 11 & 9 & 27 & 28 \\
2 & 0 & 3 & 40 & 32 \\
3 & 5 & 6 & 32 & 25 \\
4 & 2 & 5 & 41 & 25 \\
5 & 2 & 1 & 31 & 32 \\
6 & 0 & 3 & 19 & 27 \\
N & 7 & 7 & 7 & 7
\end{tabular}

$\begin{array}{lrrrr}\text { Mean } & 3.14 & 4.28 & 31.86 & 27.86 \\ \text { SD } & 3.8 & 2.69 & 7.54 & 3.02\end{array}$


Year 1

Standardised Condition.

\begin{tabular}{|c|c|c|c|c|}
\hline \multirow[t]{2}{*}{ Client } & \multicolumn{2}{|c|}{ Sociometry } & \multicolumn{2}{|c|}{ Self-report } \\
\hline & Pre & Post & Pre & Post \\
\hline 1 & - & - & 28 & 45 \\
\hline 2 & 0 & 6 & 29 & 22 \\
\hline 3 & 14 & 4 & 45 & 46 \\
\hline 4 & 14 & 9 & 25 & 10 \\
\hline 5 & 4 & 3 & 17 & 17 \\
\hline & - & - & 49 & 50 \\
\hline N & 4 & 4 & 6 & 6 \\
\hline Mean & 8 & 5.5 & 32.17 & 31.67 \\
\hline SD & 7.12 & 2.65 & 12.30 & 17.3 \\
\hline
\end{tabular}


Year 1

Control Condition.

Client Sociometry

$\begin{array}{rrr} & \text { Pre } & \text { Post } \\ 1 & 16 & 21 \\ 2 & 9 & 16 \\ 3 & 8 & 1 \\ 4 & 0 & 0 \\ 5 & 1 & 0 \\ 6 & 4 & 21 \\ \text { N } & 6 & 6\end{array}$

$\begin{array}{lll}\text { Mean } & 6.33 \quad 9.83\end{array}$

$\begin{array}{lll}\text { SD } & 5.96 \quad 10.57\end{array}$ 
Year_2

Individualised Condition.

\begin{tabular}{|c|c|c|c|c|c|}
\hline \multirow[t]{2}{*}{ Client } & \multicolumn{2}{|c|}{ Sociometry } & \multicolumn{2}{|c|}{ Self-report } & \multirow[b]{2}{*}{ Followup } \\
\hline & Pre & Post & Pre & Post & \\
\hline 1 & 0 & 0 & 38 & 38 & 26 \\
\hline 2 & 0 & 0 & - & - & - \\
\hline 3 & 4 & 18 & 37 & 38 & 23 \\
\hline 4 & 3 & 7 & 17 & 16 & 13 \\
\hline 5 & 25 & 19 & 34 & 36 & - \\
\hline 6 & 5 & 5 & 42 & 32 & 40 \\
\hline 7 & 1 & 10 & 37 & 35 & 36 \\
\hline$N$ & 7 & 7 & 6 & 6 & 5 \\
\hline Mean & 5.43 & 8.43 & 34.17 & 32.5 & 27.6 \\
\hline SD & 8.85 & 7.76 & 8.80 & 8.32 & 10.73 \\
\hline
\end{tabular}




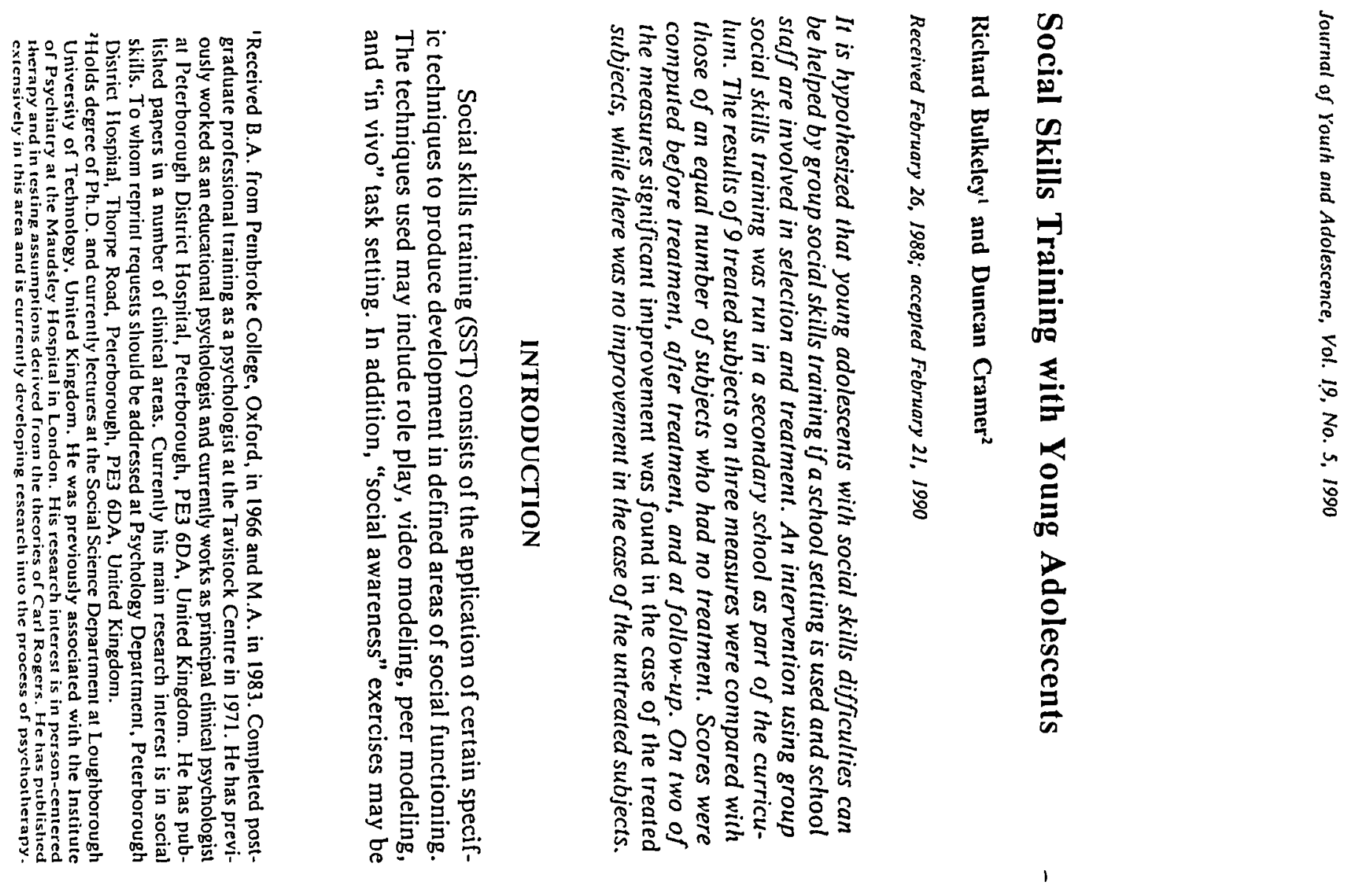




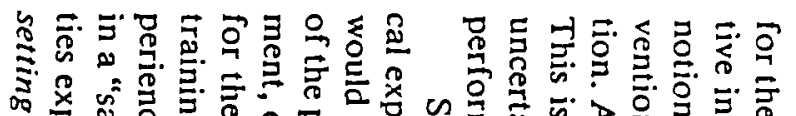

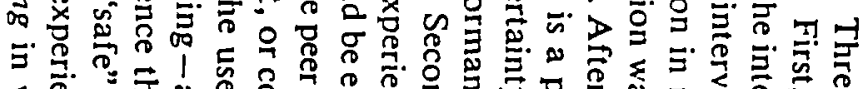

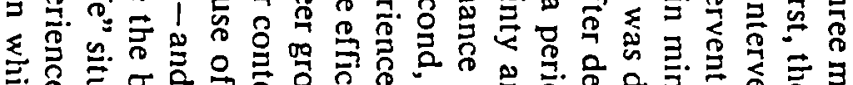
훙영.

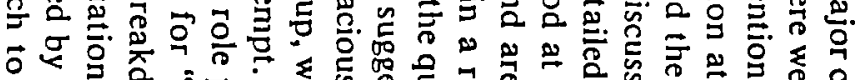

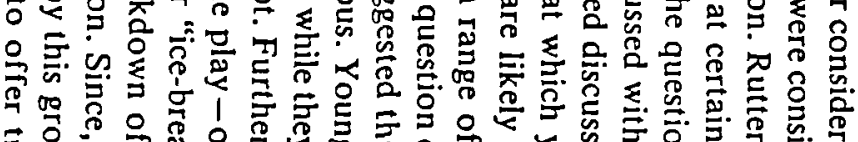

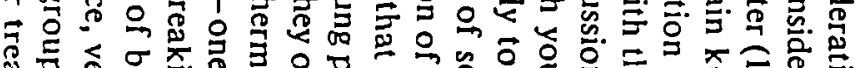

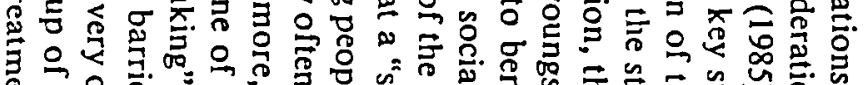

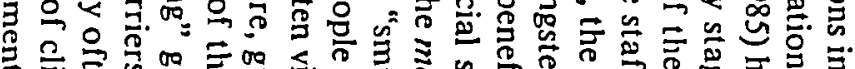

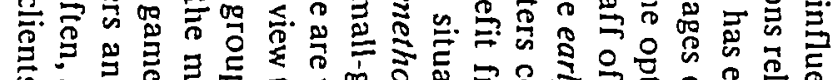

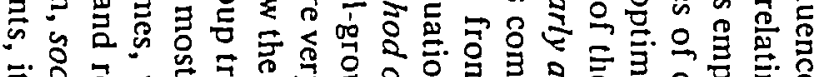

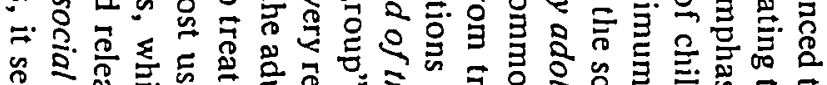

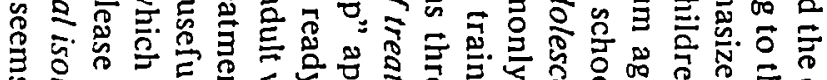

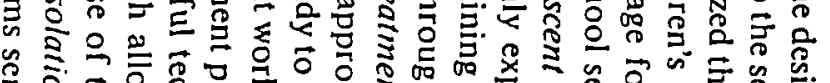

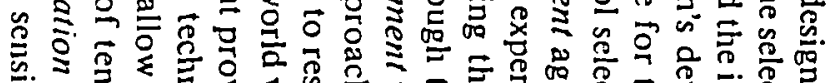

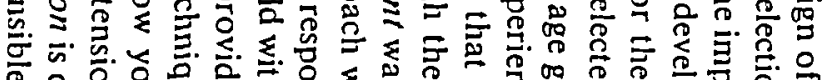

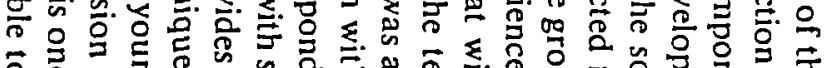

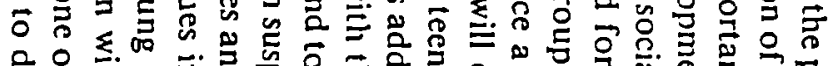

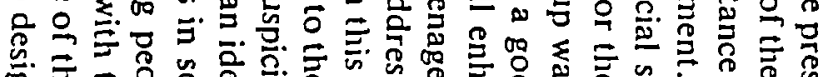

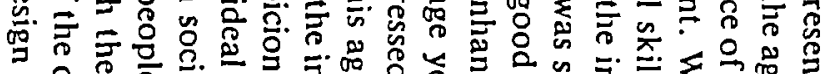

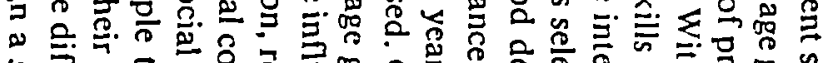

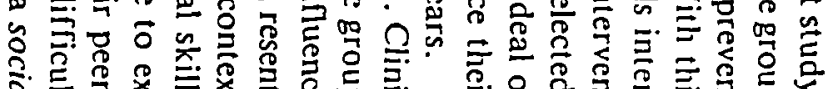

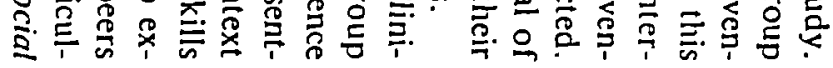

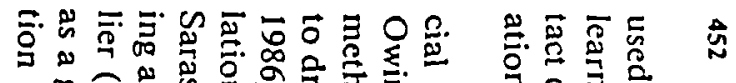

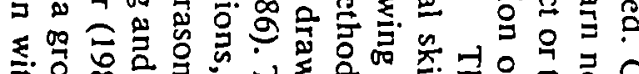

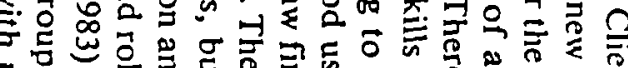

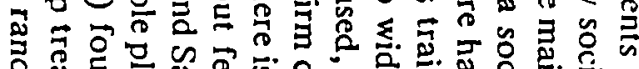

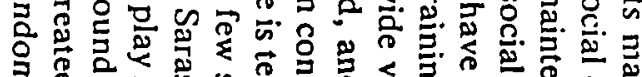

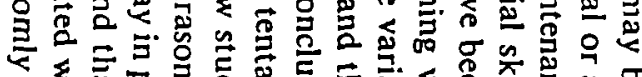

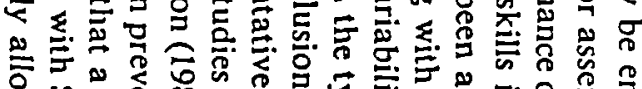

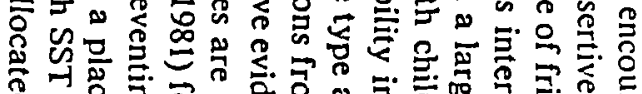

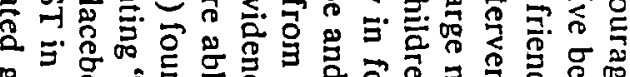
물

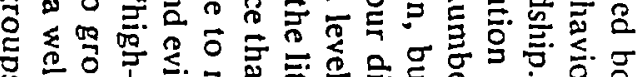

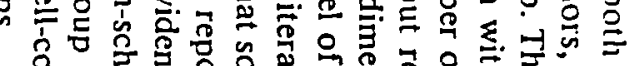

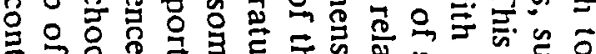

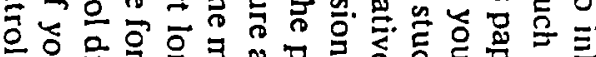

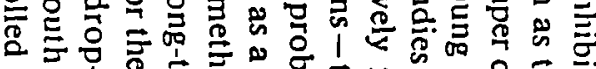

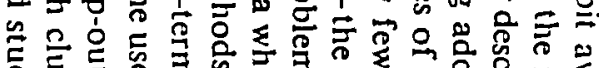
20

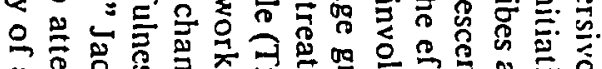

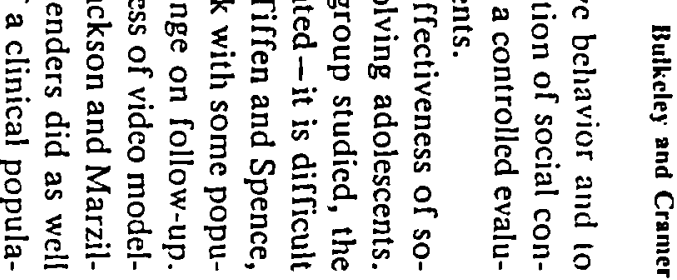

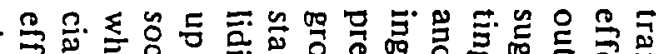

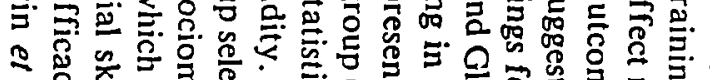

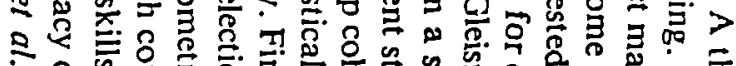
은

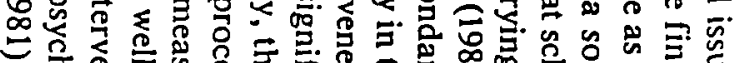

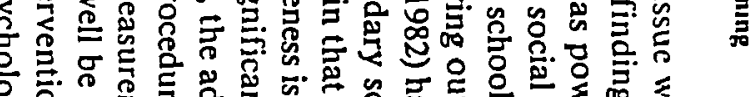

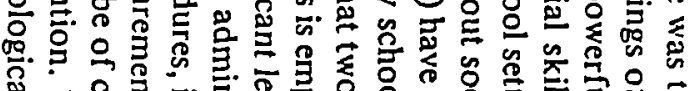

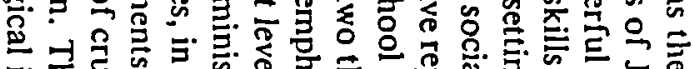
F者

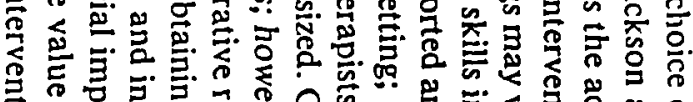

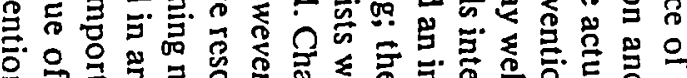
을

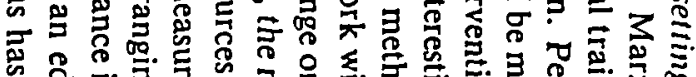

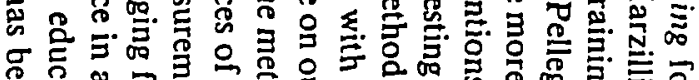
象

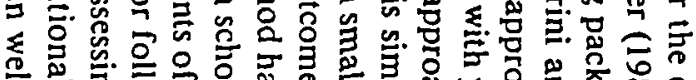

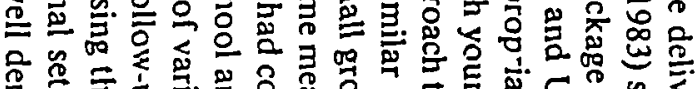

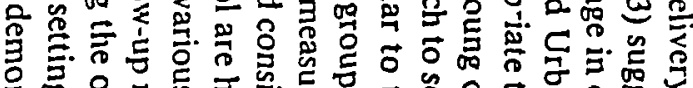

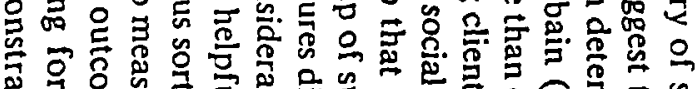

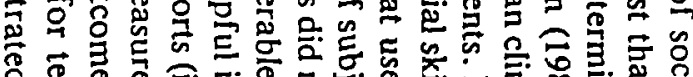

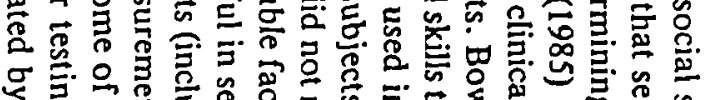

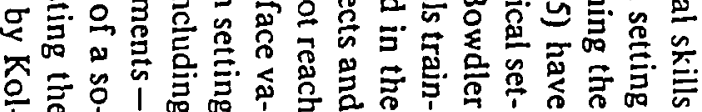



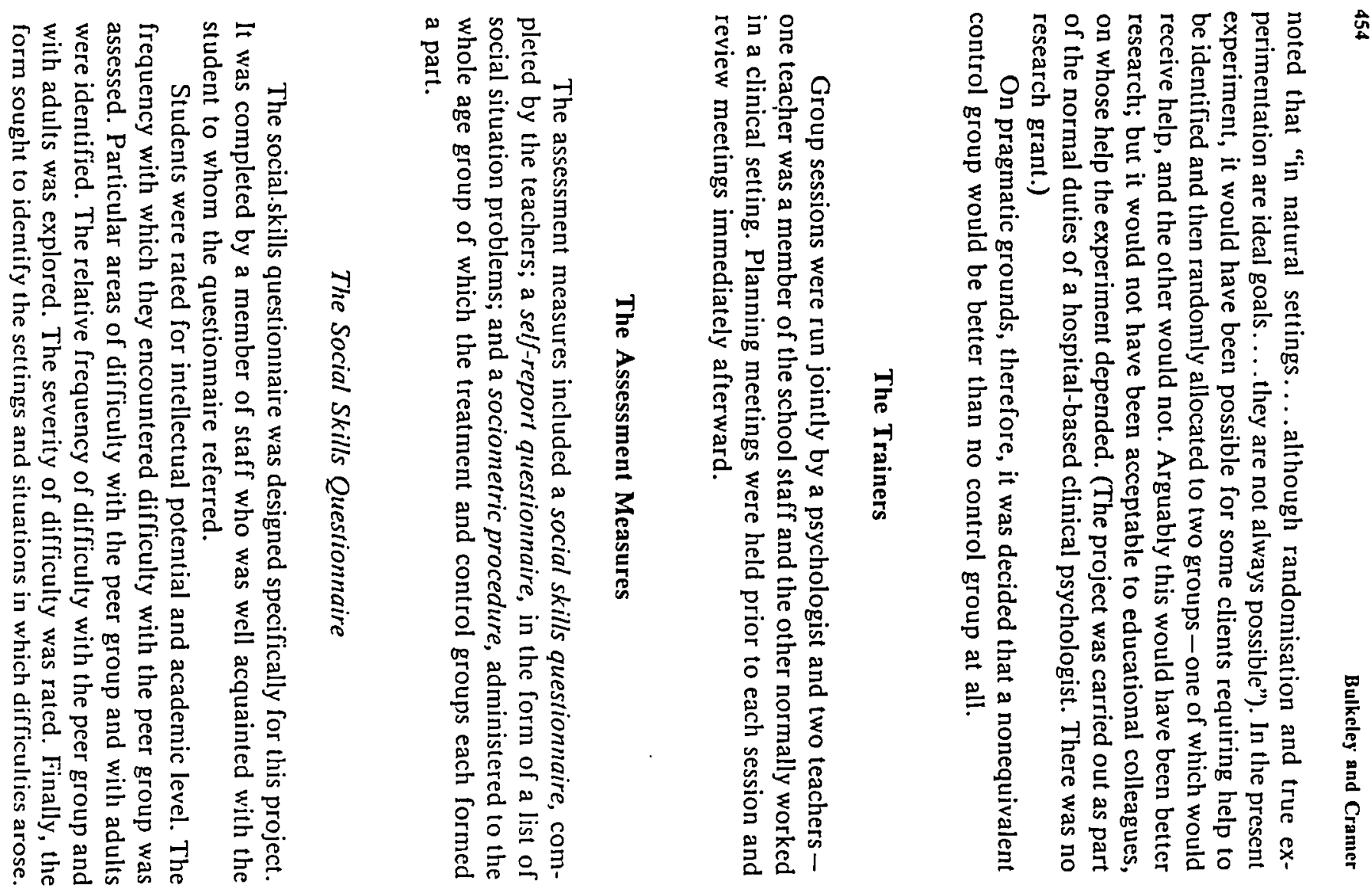

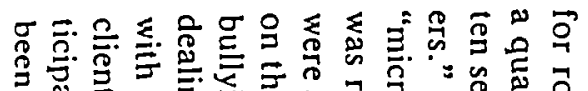

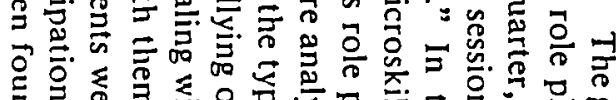

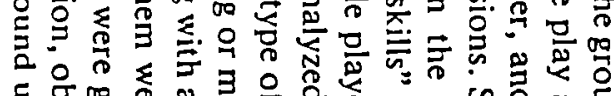

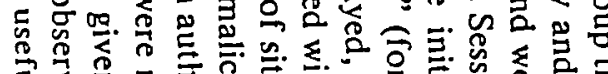

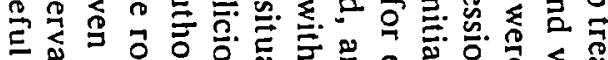

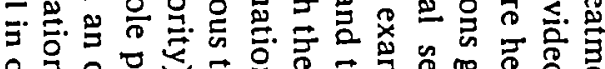

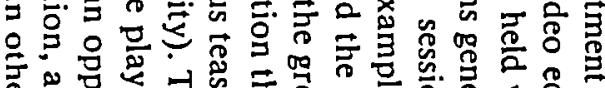

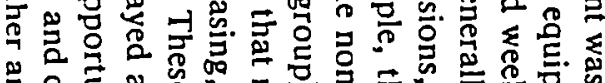

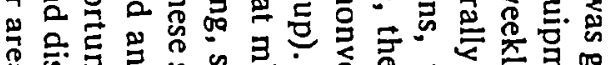

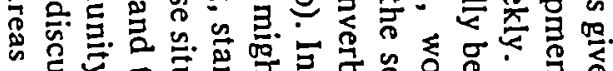

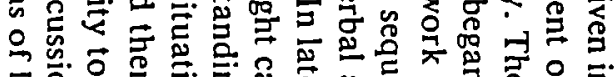

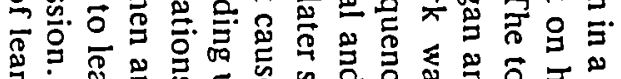

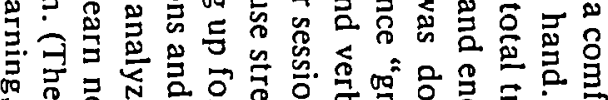

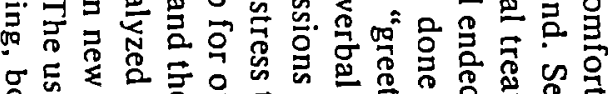

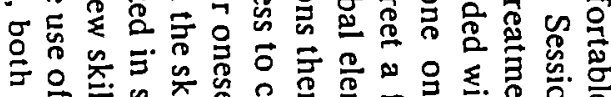

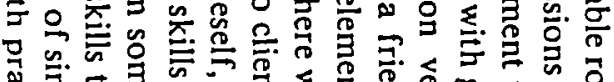

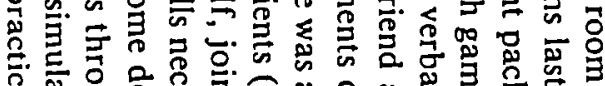

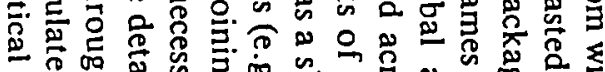

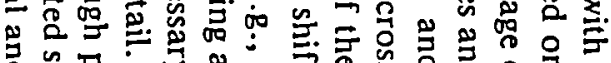

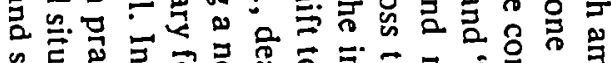

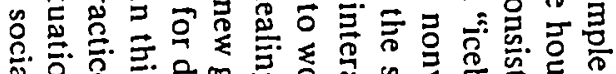

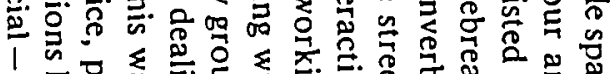

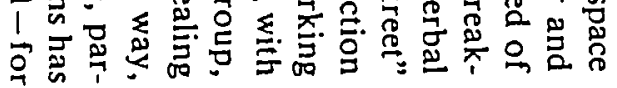

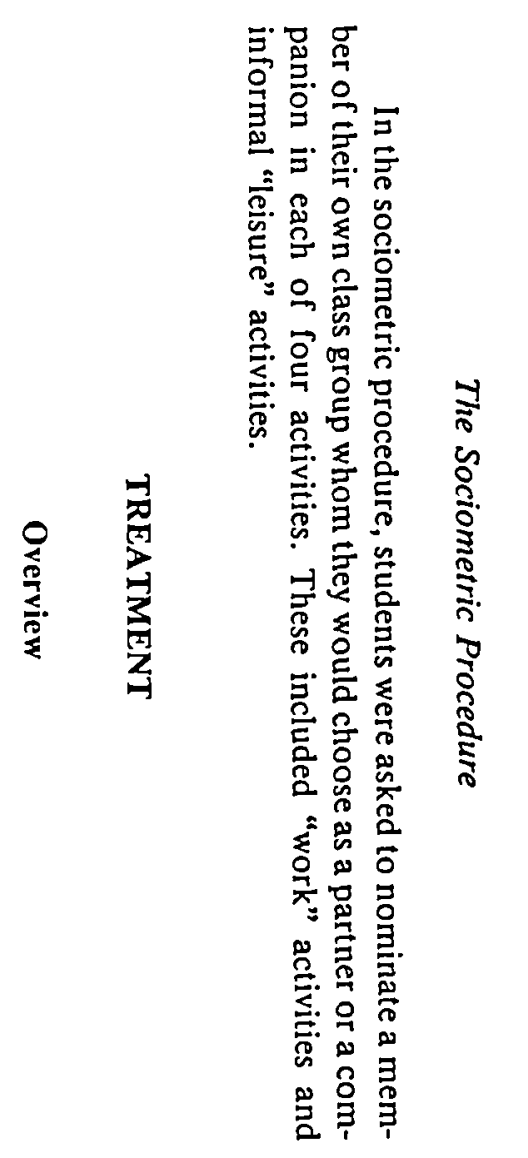

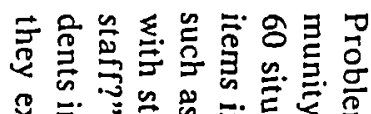

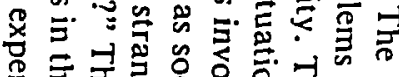

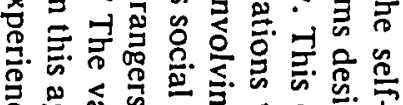

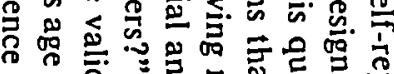

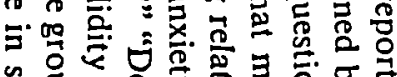

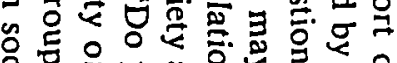

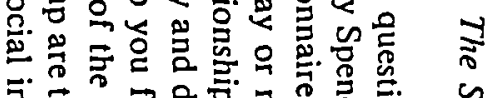

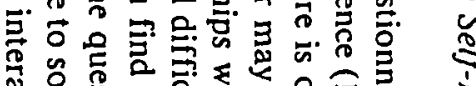

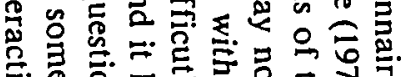

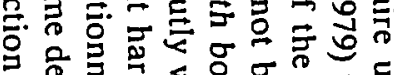

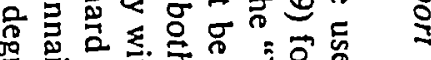

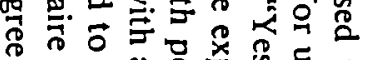

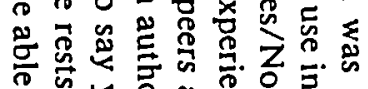

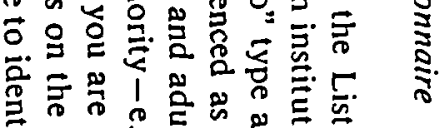

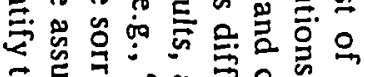

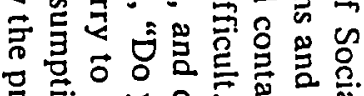

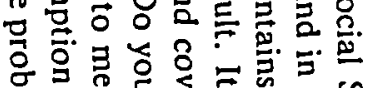

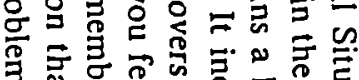

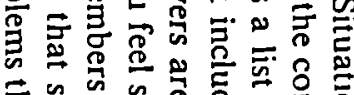

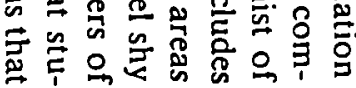




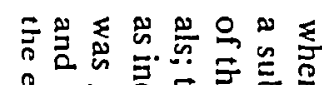

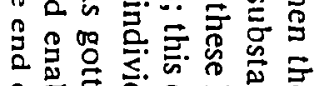

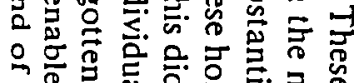

으의.

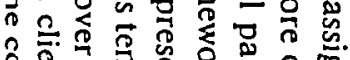

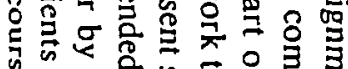

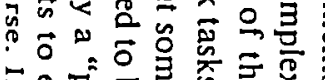

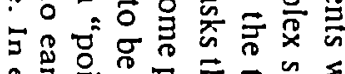

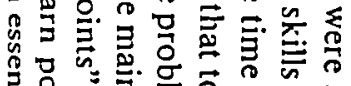

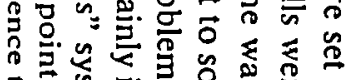

言自然言

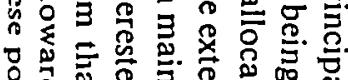

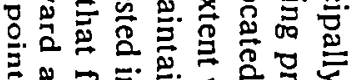

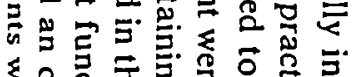

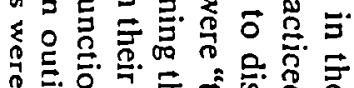

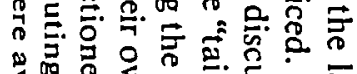

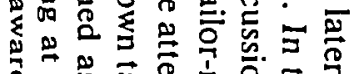

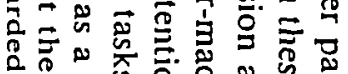

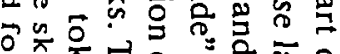

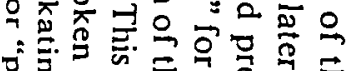

호ำ

然

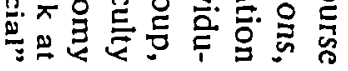

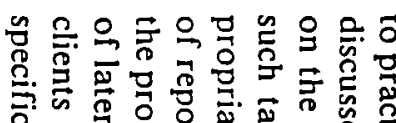

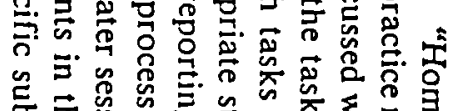

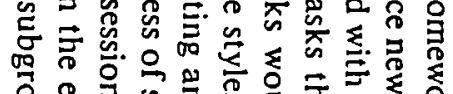
을

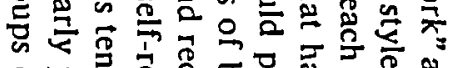

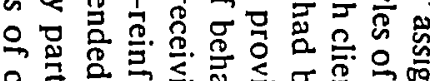

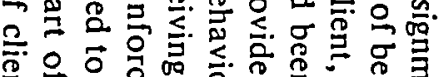
蛋

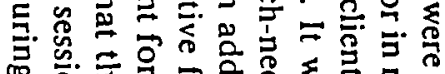

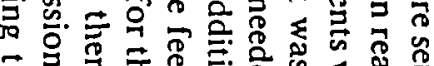

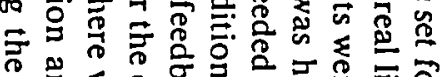

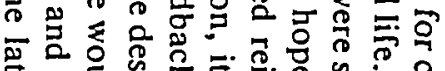

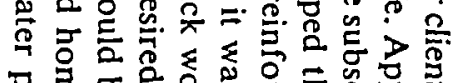

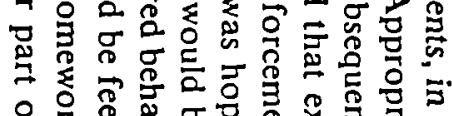

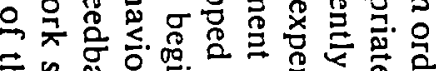

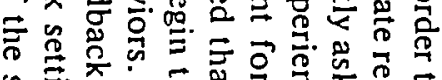

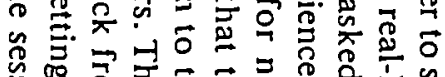

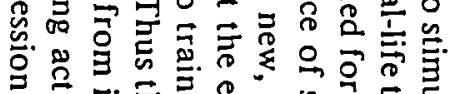

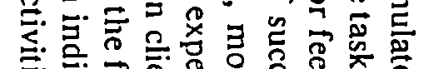

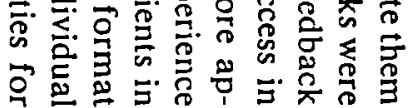

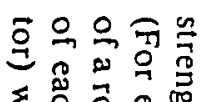

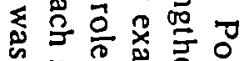

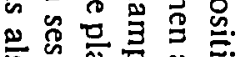

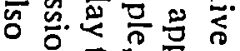

约

웅

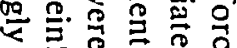

要

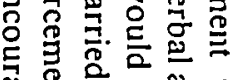

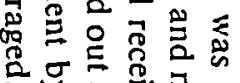

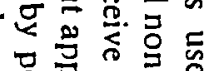

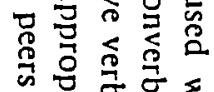

के 콩.

常可蛋

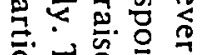

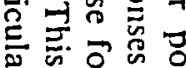

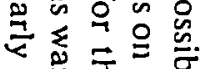

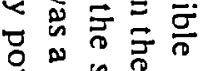

话㶽可

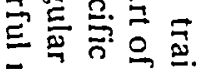

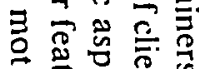

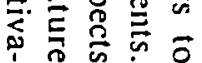

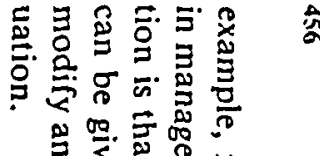

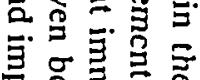

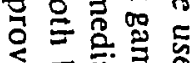

त

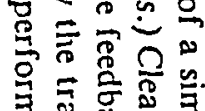

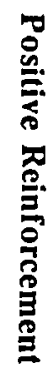

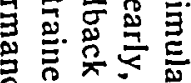

을

․ㅡㄹ.

可高票

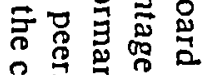

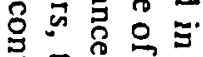

要要要

品言空.

는

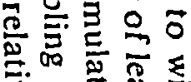

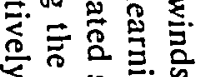

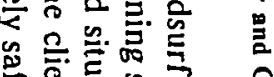

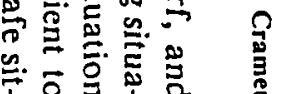

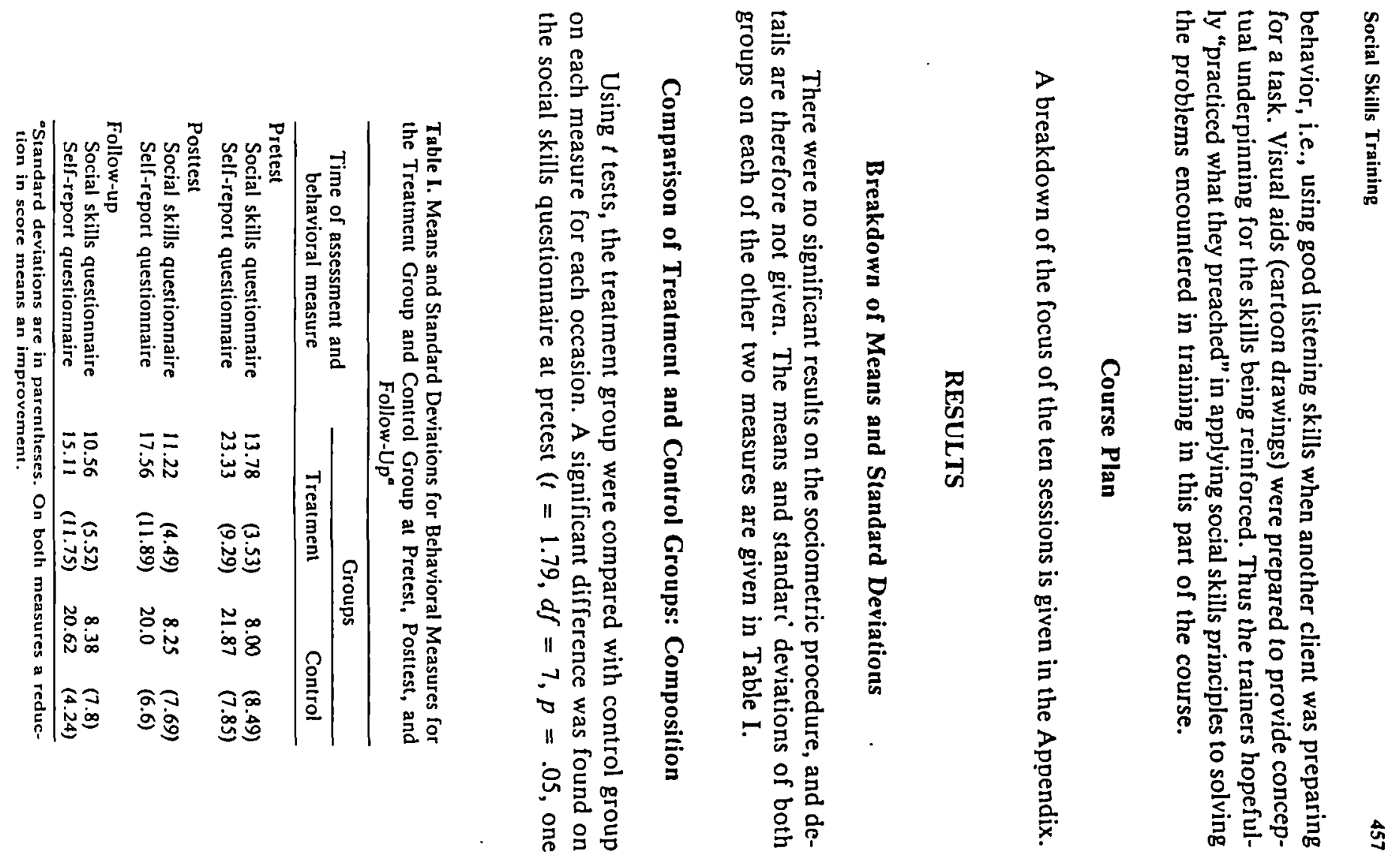




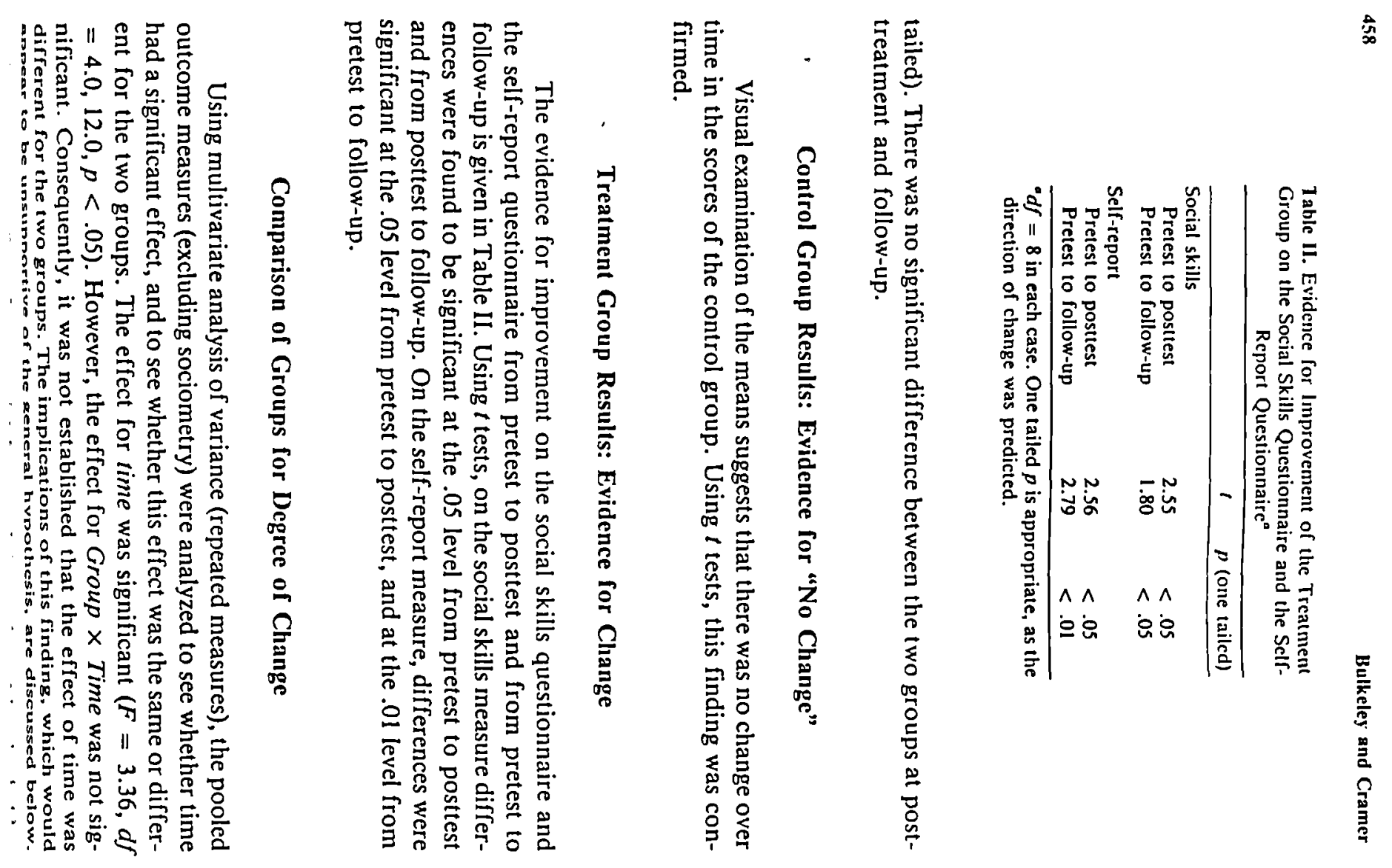

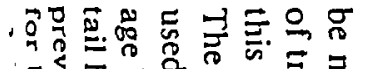

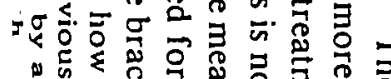

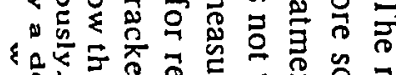

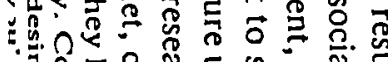

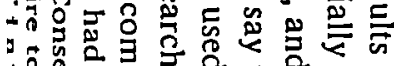

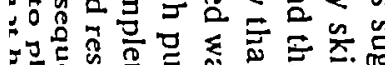

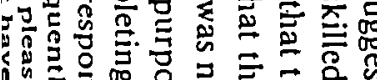

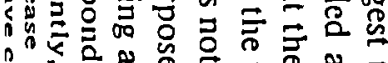

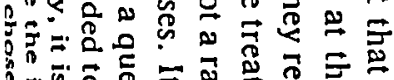

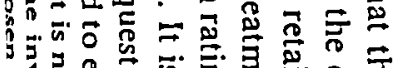

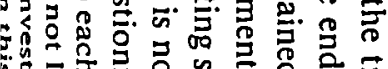

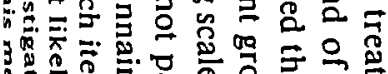

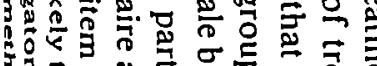

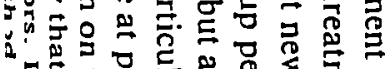

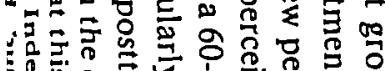

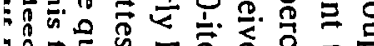

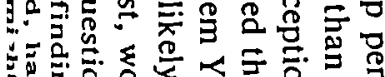

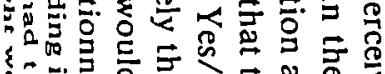

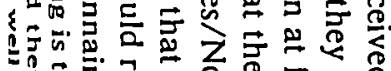

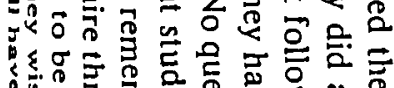

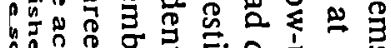

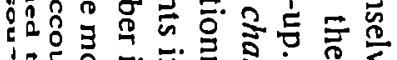

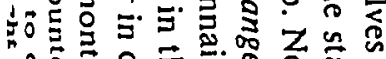
की०

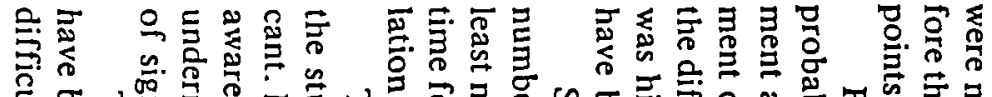

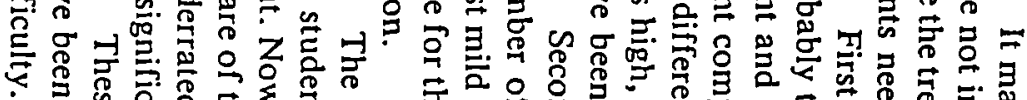

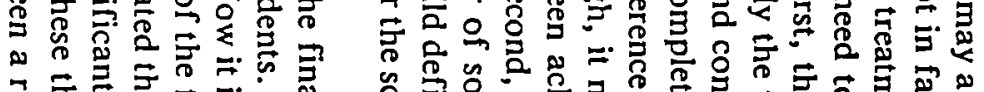

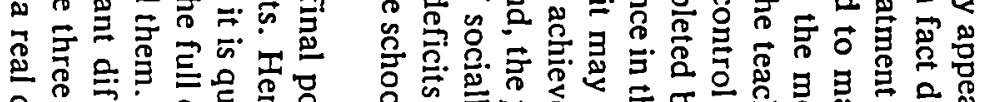

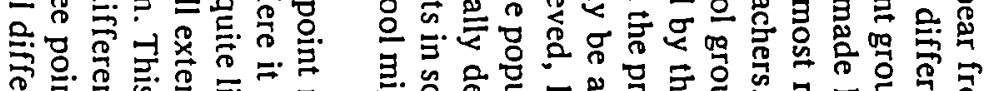

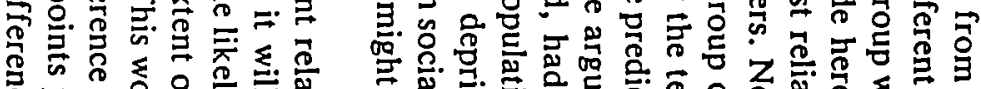

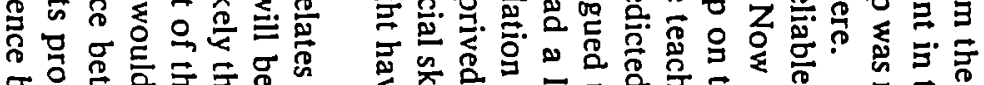

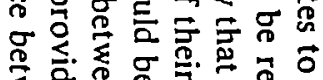

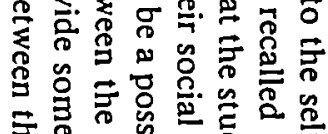

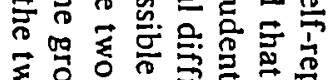

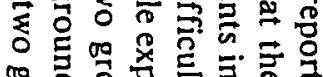

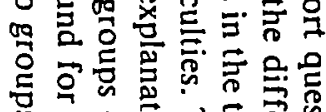

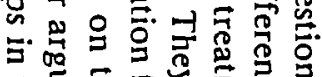

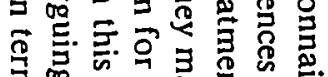

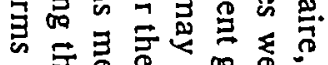

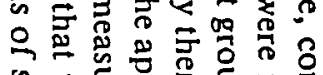

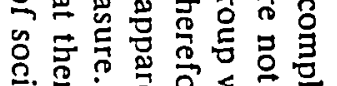

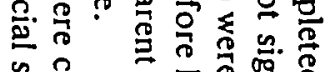

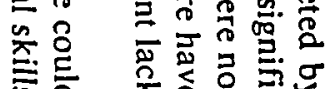

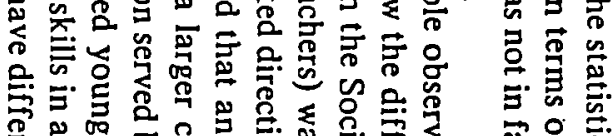

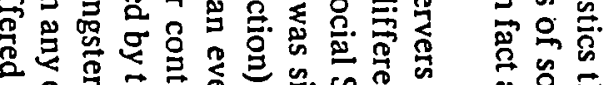

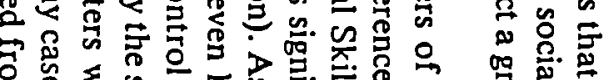

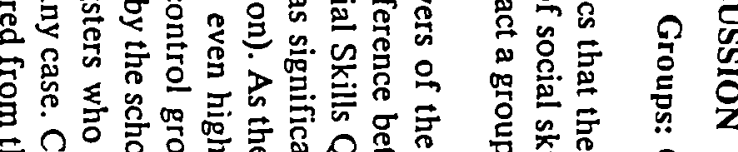

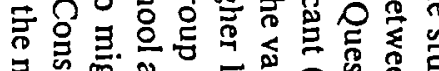

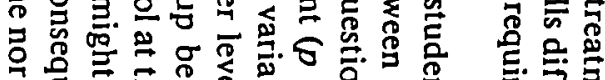

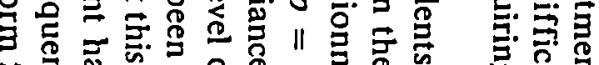

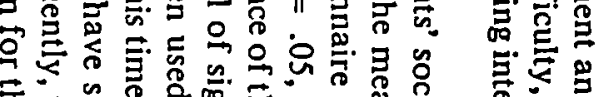

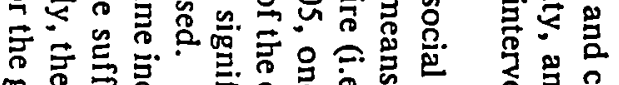

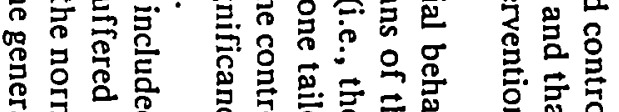
总寻员命 굴훙ㅁㅇㅇ 

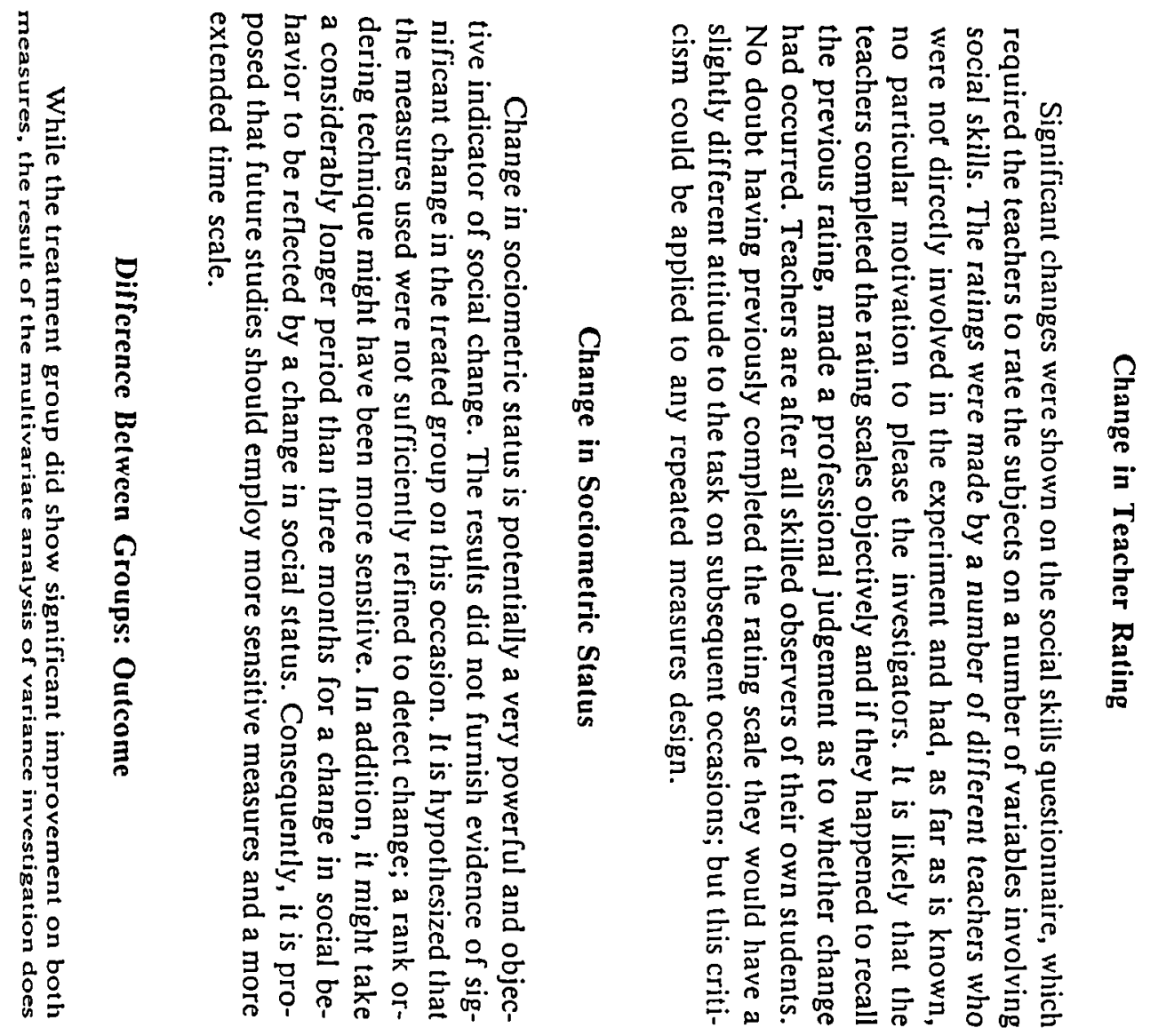

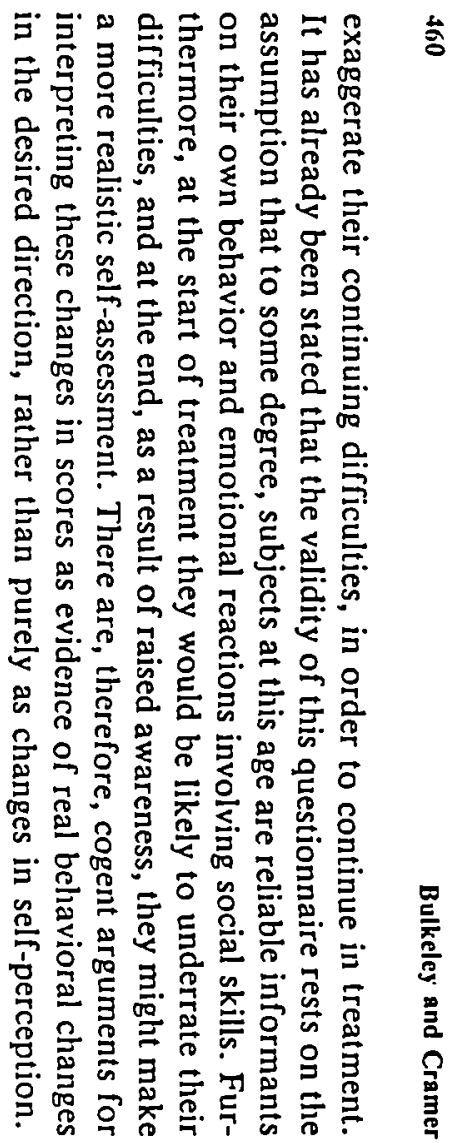

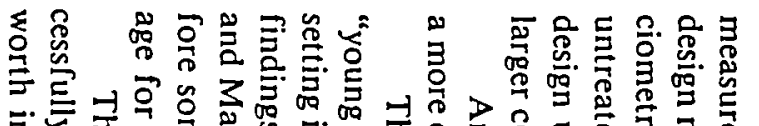

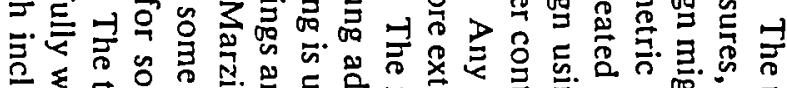

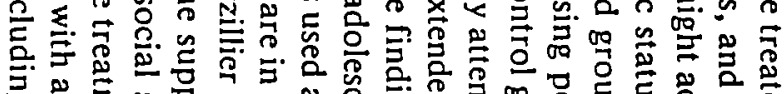

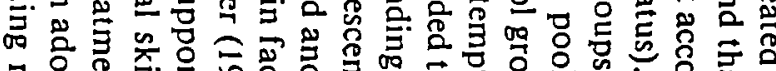

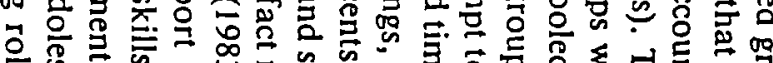

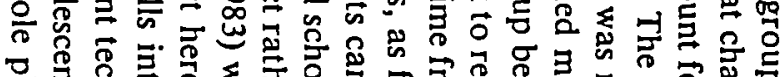

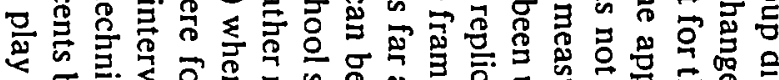

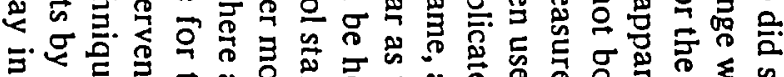

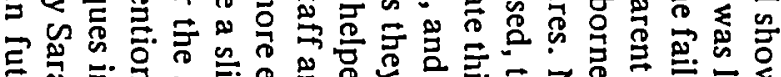

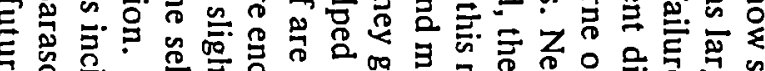

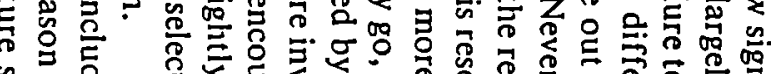

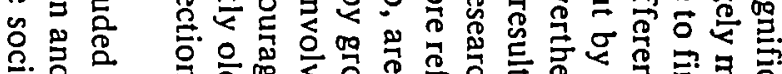

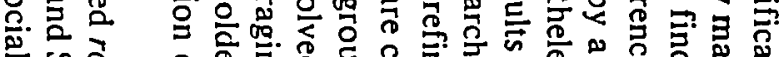

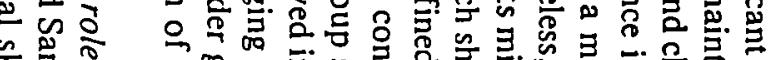

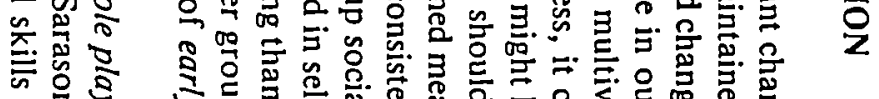

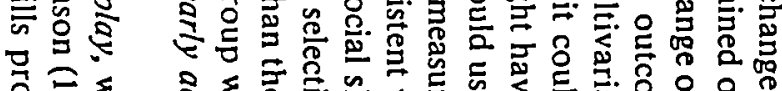

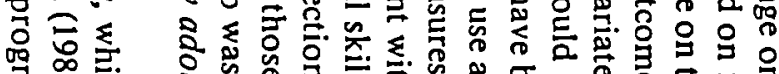

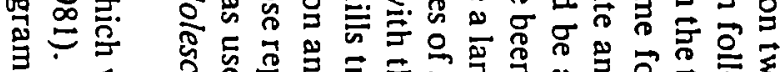

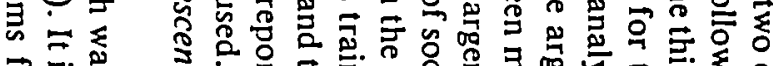

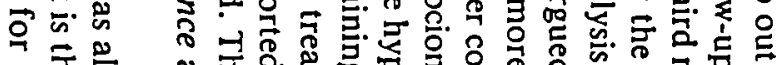

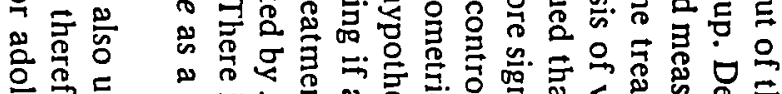

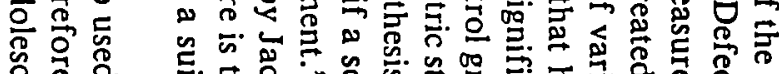

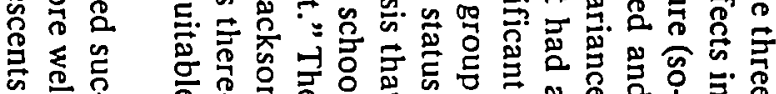

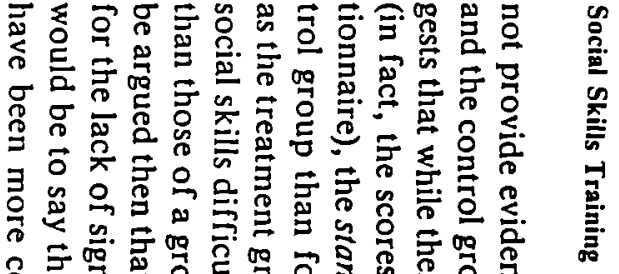

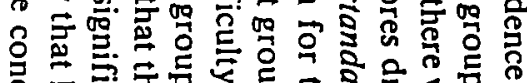

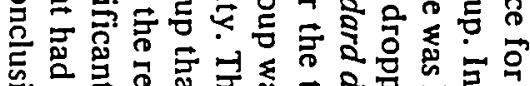

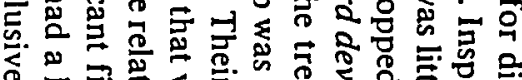

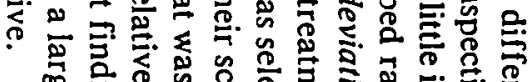

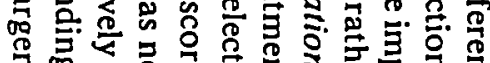

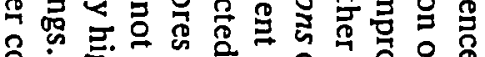

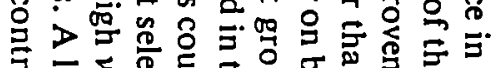

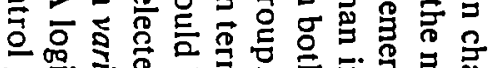

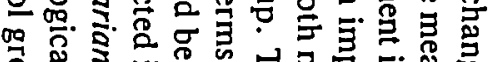
음

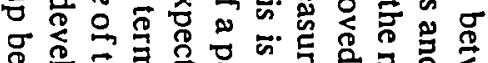

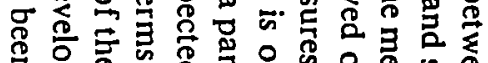

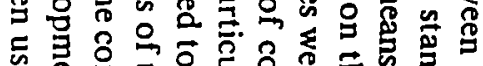

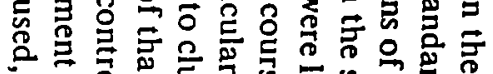

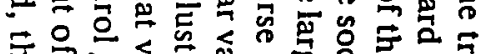

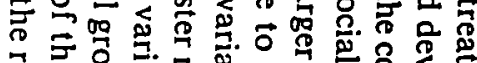

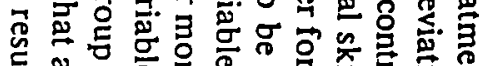

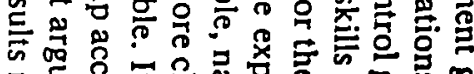

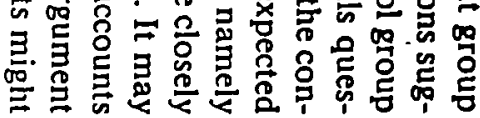



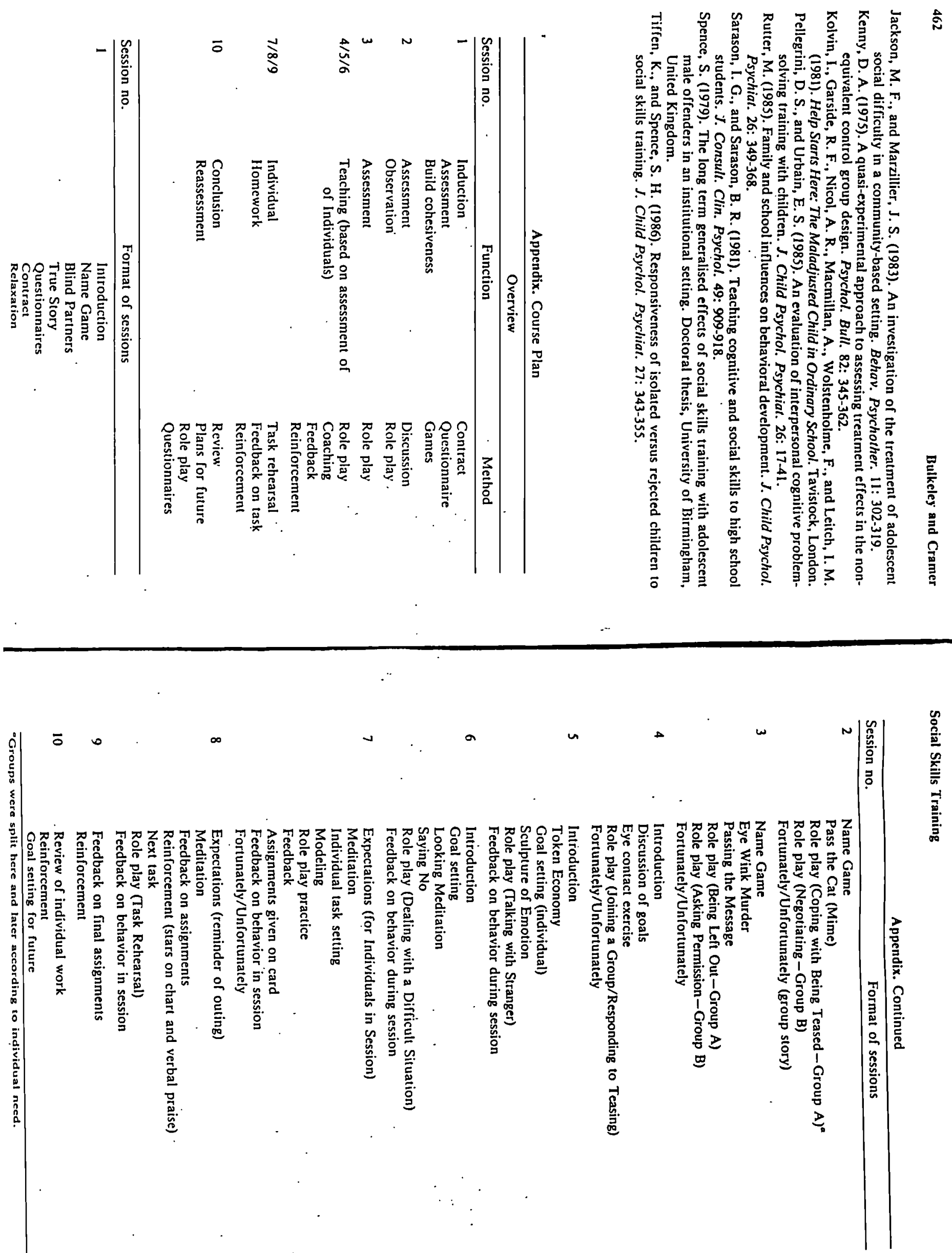
SOCIAL SKILLS TRAINING WITH YOUNG ADOLESCENTS

Chapter Six

References 
SOCIAL SKILLS TRAINING WITH YOUNG ADOLESCENTS

Chapter Six

\section{References}

Alexander, J. F. \& Parsons, B. V. (1973). Short-term behavioural intervention with delinquent families: Impact on family processes and recidivism. Journal of Abnormal Psychology, 81, 219-225.

Arqyle, M. (1967). The psychology of interpersonal behaviour. Harmondsworth: Penquin Books.

Argyle. M. (1977). Predictive and qenerative rule models of $P \quad x \quad S$ interaction. In D. Maqnusson \& N. S. Endler (Eds.) Personality at the crossroads: Current issues in interactional psychology. Hillside, N.J: Erlbaum.

Argyle, M., \& Kendon., A. (1967). The experimental analysis of social performance. In Advances in Experimental Social Psychology (vol.3). New York \& London: Academic Press.

Asher, S. R., Markell, R. A. \& Hymel, S. (1981). Identifying children at risk in peer relations: $\AA$ critique of the rate-of-interaction approach to assesment. Child Development, 52, 1239-1245.

Asher, S.R. (1983). Social competence and peer status: Recent advances and future directions. Child Development, 54, 1427-1434.

Asher, S.R. \& Renshaw. P.D. (1981). Children without friends: Social knowledge and social skills trainina. In S.R.Asher \& J. M. Gottman (Eds.) The development of children's friendships. New York: Cambridge University Press. 
Bandura, A. (1969). Principles of behaviour modification. New York: Holt, Rinehart \& Winston.

Bandura, A. (1977a). Self-efficacy: toward a unifyina theory of behavour change. Psychological Review,84, $191-215$.

Bandura, A. (1977b). Social learning theory. Englewood Cliffs, N.J: Prentice-Hall.

Barrett. C. L. (1978). Research on psychotherapy with children. In S. L. Garfield \& A. E. Bergin (Eds.) Handbook of psychotherapy and behaviour change: an empirical analysis. New York: Wiley.

Beck, S. \& Forehand, R. (1984). Social skills training for children: A methodological and clinical review of behaviour modification studies. Behavioural Psychotherapy, 12, 17-45.

Beck, S., Forehand, R., Neeper. R \& Baskin, C.H. (1980). A comparison of two analogue strateqies for assessing children's social skills. Journal of Consulting and Clinical Psychology, 50, 598-602.

Bellack, A. S. (1983). Recurrent problems in the behavioural assessment of social skills. Behaviour Research and Therapy, 21, 29-42.

Bellack, A. S., Hersen, M. \& Lamparski, D. $(19 \% 9)$. Role-play tests for assessing social skills: Are they valid? Are they useful? Journal of Consulting and Clinical Psychology, 47, 335-342.

Bellack, A. S., Hersen, M. \& Turner, S. M. (1978). Role-play tests for assessing social skills: Are they valid? Behaviour Therapy, 9, 448461 .

Berman, J. S. \& Norton, N. C. (1985). Does professional training make a therapist more effective? Psychological Bulletin, 98, 401-407. 
Bonner, R. L. \& Rich, A. (1988). Neqative life stress, social problemsolving self-appraisal, and hopelessness: Implications for suicide research. Coonitive Therapy and Research, 12, 549-556.

Bornstein, M. R., Bellack, A. S. \& Hersen, M. (1977). Social-skills training for unassertive children: A multiple baseline analysis. Journal of Applied Behaviour Analysis, 10, 183-195.

Bowdler. D. \& Gleisner, S. (1982). Killer winks in the secondary school? A personal and social skills course. Association for Educational Psychologists Journal, 5, 64-69.

Bryant, B. \& Trower, P.E. (1974). Social difficulty in a student sample. British Journal of Educational Psychology, 44, 13-21.

Buell, $G$ \& Snyder, J (1981). Assertiveness training with children. Psychological Reports, 49, 71-80.

Callias, M. \& Likierman, H. The social cognition and concept of friendship test. Unpublished manuscript. Available from M. Callias, Department of Psychology, Institute of Psychiatry, De Crespigny Park, London SE5 8AF.

Chandler, M. J. (1973). Eqocentrism and antrsocial behaviour: The assessment and training of social pespective-taking skills. Developmental Psychology, 9, 326-332.

Coie, J. D. \& Kupersmidt, J. B. (1983). A behavioural analysis of emerging social status in boys groups. Child Development, 54, 1400-1416.

Conger, J. C. \& Keane, S. P. (1981). Social skills intervention in the treatment of isolated or withdrawn children. Psychological Bulletin, $90, \quad 478-495$.

Conning, A. M. \& Head, D. M. (1990). Friends - Who needs them? Two case studies illustating the assessment and treatment of boys with peer relationship difficulties. Behavioural Psychotherapy, 18, 221-233. 
Coopersmith, S. (1967). The antecedents of self-esteem. San Francisco: Freeman.

Craighead, W. E., Kazdin, A. E. \& Mahoney, M. J. (1976). Behaviour modification: Principles, issues, and applications. Boston: Houghton Mifflin.

Crick, N. R. \& Ladd, G. W. (1990). Children's perceptions of the outcomes of social strateaies: Do the ends justify being mean? Developmental Psychology, 26, 612-620.

Cowen, E. L., Pederson, A.. Babigian, H., Izzo, L. D. \& Trost, M.A. Longterm follow-up of early detected vulnerable children. Journal of Consulting and Clinical Psychology, 41, 438-446.

Cox, R. D., Gunn, W. B. \& Cox, M. J. (1976). A film assessment and comparison of the social skilfulness of behaviour problem and nonproblem male children. Paper presented at a meting of the Association for the Advancement of Behaviour Therapy, New York, December 1976.

Curran, J. P. (1979). Social skills: Methodological issues and future directions. In A. S. Bellack and M. Hersen (EdS.) Research and practice in social skills trainina. New York: Plenum Press.

Curran, J. P., Farrell, A. D., \& Grunberger, A. J. (1984). Social skills: A critique and a rapprochement. In P. Trower (Ed.) Radical approaches to social skills training. London: Croom Helm.

Dodge, K. A. (1980). Social cognition and children's aqgressive behaviour. Child Development, 51, 162-170.

Dodge, K. A. (1983). Behavioural antecedents of peer social status. Child Development, 54, 1386-1399.

D'Zurilla, T. J. \& Goldfried, M. R. (1971). Problem solving and behaviour modification. Journal of Abnormal Psychology, 78, 107-126. 
Dubow, E.F.. Huesmann, L.R.,\& Eron, L.D. (1987). Mitigating aggression and promoting prosocial behaviour in aqaressive elementary schoolboys. Behaviour Research and Therapy, 25, $527-531$.

Ekman, P. \& Friesen, W. V. (1969). The repertoire of non-verbal behaviour: Cateqories, oriqins, usaqe and coding. Semiotica, 1, 49-98.

Ekman, P. (1971). Universals and cultural differences in facial expressions of emotion. In J. Cole (Ed.) Nebraska symposium on motivation. Lincoln: University of Nebraska Press.

Emery, R.E. \& Marholin. D. (1977). An applied behaviour analysis of delinquency. American Psycholog̣ist, 6, $860-873$.

Endler, N. \& Maqnusson, D. (1976). Towards an interactional psychology of personality. Psychological Bulletin, 33, 956-974.

Farrell, M. (1980). An overview of the social skills model as used with young teenagers. Paper given at Ida Darwin Hospital, Cambridge.

Feffer, M. \& Gourevitch, V. (1960). Coonitive aspects of roletaking in children. Journal of Personality, 28, 384-396.

Filipczak, J.. Archer, M. \& Friedman, R.M (1980). In-school social skills training; Use with disruptive adolescents. Benaviour Modification, 4 , $243-263$.

Forqas, J. P. (1983). Social skills and the perception of interaction episodes. British Journal of Clinical Psychology, 22, 195-207.

Foster, S. F. \& Ritchey, W. L. (1979). Issues in the assessment of social competence in children. Journal of Applied Behaviour Analysis, 12, 625638 .

Frank, J. D. (1973). Persuasion and Healing: a comparative study of psychotherapy. (Rev. ed.) Baltimore: Johns Hopkins University Press. 
Freedman, B. J., Rosenthal. L., Donahoe C. P., Schlundt, D.G . \& McFall, R. M. (1978). A social-behavioural analysis of skills deficits in delinquent and nondelinquent adolescent boys. Journal of Consulting and Clinical Psychology, $46,1448-1462$.

French, D. C. \& Tyne, T. F. (1982). The identification and treatment of children with peer-relationship difficulties. In J. P. Curran \& P. M. Monti (Eds.) Social skills training: A practical handbook for assessment and treatment. New York: Guildford Press.

Frosh, S.R. (1983). Children and teachers in schools. In S. Spence and G. Shepherd (Eds.) Developments in social skills training. London: Academic Press.

Furman, W., Rahe, D. F. \& Hartup, W. W. (1979). Rehabilitation of a socially-withdrawn pre-school child through mixed-age and same-age socialisation. Child Development, 50, 915-922.

Furnham, A. (1986). Social skills training with adolescents and young adults. In C. R. Hollin \& P. Trower (Eds.) Handbook of social skills training : (Vol. 1) Applications across the life span.

Gaffney, L. R. \& MCFall, R. M. (1981). A comparison of social skills in delinquent and non-delinquent adolescent girls using a behavioural roleplaying inventory. Journal of Consulting and Clinical Psychology, 49, $959-967$.

Gallassi, J., Gallassi, M. \& Vedder, M. (1981). Perspectives on assertion as a social skills model. In $T$. Wine and M. Smye (Eds.) Social Competence. New York: Guildford Press.

Goetz, T. E. \& Dweck, C. S. (1980). Learned helplessness in social situations. Journal of Personality and Social Psychology, 39, 246-255. Goffman, E., 1972. Relations in public: Microstudies of the public order. Harmondsworth: Penguin. 
Goldman, J. A., Corsini, D. A. \& Deurioste, R. (1980). Implications of positive and neqative sociometric status for assessing the social competence of young children. Journal of Applied Developmental Psychology, 1, 209-220.

Goldstein. A. P., Lopez. M. \& Greenleaf. D.0 (1979). Introduction. In A. P. Goldstein \& F. H. Kanfer (Eds.) Kaximising Treatment Gains. New York: Academic Press.

Gottman, J., Gonso, J.. \& Rasmussen, B. (1975). Social interaction, social competence, and friendship in children. Child Development, 46, 179-197. Green, K. D., Forehand, F., Beck, S. J. \& Vosk, B. (1980). An assessment of the relationship among measures of children's social competence and children's academic achievement. Child Development. 51, 1149-1156.

Gresham, F. M. (1981). Validity of social skills measures for assessing social competence in low status children: A multivariate investigation. Developmental Psychology, 17, 390-398.

Gross, A. M., Brigham, T. A., Hopper, C. \& Bologna, N. C. (1980). Selfmanagement and social skills training: A study with pre-delinquent and delinquent youths. Criminal Justice and Behaviour, 7, 161-184.

Guerra, N. G. \& Slaby, R. G. (1990) Cognitive mediators of aggression in adolescent offenders: 2. Intervention. Developmental Psychology, 26, $269-277$.

Ha11. E.T., (1963). A system for the notation of proxemic behaviour. American Anthropology, 65, 1003-1026.

Hal1, E.T. (1964). Silent assumptions in social communication. Res. Publ. Assoc. Nerv, Ment. Dis., 42, 41-5.

Hammen, C. L., Jacobs, M., Mayol, A.. \& Cochran, S. D. (1980). Dysfunctional cognitions and the effectiveness of skills and cognitivebehavioural assertion training. Journal of Consulting and clinical Psychology, 48, 685-695. 
Hartup, W., Glazer, J. \& Charlesworth, R. (1967). Peer reinforcement and sociometric status. Child Development, 38, 1017-1024.

Hazel, J. S., Schumaker, J. B., Sherman, J. A. \& Sheldon-Wildgen, J. (1981). The development and evaluation of a aroup skills training procram for court-adjudicated youth. In D. Upper and S. M. Ross (Eds.) Behaviour group therapy, 1981: a review. Champaign, Illinois: Research Press.

Heimberg, R., Cunninqham., J., Stanley. J., \& Blacenberq, R. (1982). Social skills training to prepare unemployed youth for the job interview. Behaviour Modification, $6,299-322$.

Henderson, M. \& Hollin, C. (1983). A critical review of social skills training with young offenders. Criminal Justice and Behaviour, 10, 316341.

Henderson, M. \& Hollin, C. (1986). Social skills training and delinquency. In C. R. Hollin \& P. Trower (Eds.) Handbook of Social Skills Training. (Vol 1). Applications across the life span. Oxford: Pergamon.

Herbert, M. (1986), Social Skills Training with Children. In C. R. Hollin \& P. Trower (Eds.) Handbook of social skills training. (Vol.1). Applications across the lifespan. Oxford: Pergamon.

Hollin, C. R. \& Trower, P. (Eds.) Handbook of social skills training. (Vols. $1 \& 2)$. Oxford: Pergamon.

Hollin, C.R. \& Henderson, M. (1984). Social skills training with young offenders: False expectations and the "Failure of treatment". Behavioural Psychotherapy, 12, 331-341.

Hood, E., Lindsay., W., \& Brooks., N. (1982). Interview training with adolescents. Behaviour Research and Therapy, 20, 581-592.

Hops, H. (1983). Children's social competence and skill: Current research practices and future directions. Behaviour Therapy, 14, 3-18. 
Hudson, B. L. (1986). Community applications of social skills training. In C. R. Hollin and P. Trower (Eds) Handbook of socisl skills training. Oxford: Pergamon.

Hymel, S. \& Asher, S. R. (1977). Assessment and training of isolated children's social skills. Paper presented at the biennial meeting of the Society for Research in Child Development, New Orleans. (ERIC Documentation Reproduction Service, No ED 136-930).

Jackson, M. F. \& Marzillier, J. S. (1983). An investiqation of the treatment of adolescent social difficulty in a community-based setting. Behavioural Psychotherapy, 11, 302-319.

Joos, M. (1962). The five clocks. International Journal of American Linguistics, 28, (2) (Part 5).

Jones, W., Hobbs, S. \& Hockenbury, D. (1982). Loneliness and social skills deficits. Journal of Personality and Social Psychology, 42, 682689.

Jones, E.E. \& Nisbett, R.E. (1971). The actor and the observer: Divergent perceptions of the causes of behaviour. Morristown, N.J: General Learning.

Jones, E.E., \& Thibaut, J.W., (1958). Interaction goals as bases for inference in interpersonal perception. In R. Tagiuri \& L. Petrullo (Eds.) Person perception and interpersonal behaviour. Stanford, California; Stanford Univ. Press.

Jung, C. G. (1934) The state of psychotherapy today. Collected Works, Vol. 10. Civilisation in transition. NJ: Princetown University Press. 1964, pp 157-173.

Kagan, C. (1984). Social problem solving and social skills training. British Journal of Clinical Psychology, 23, 161-173. 
Kazdin, A. E., Esveldt-Dawson, K. \& Matson, J. L. (1982). Changes in childrens social skills performance as a result of preassessment experiences. Journal of Clinical Child Psychology, 11, 243-248.

Kazdin, A. E., Esveldt-Dawson, K. \& Matson, J. L. (1983). The effects of instructional set on social skills performance among psychiatric inpatient children. Behaviour Therapy, 14, 413-423.

Kelly, G. (1955). The Psychology of personal constructs. New York: Norton.

Kenny, D. A. (1975). A quasi-experimental approach to assessing treatment effects in the non-equivalent control groud desian. Psychological Bulletin, $82,345-362$.

Ladd, G. W. \& Asher, S. R. (1985). Social skills training and children's peer relations. In L. L'Abate \& M.A. Milan (Eds.) Handbook of social skills training and research. New York: Wiley.

La Greca, A. M. \& Santogrossi, D. A. (1980). Social skills training with elementary school students: a behavioural group approach. Journal of Consulting and Clinical Psychology, 48, 220-228.

Liberman, R. P., Kinq, L. W., Derisi, W. J. \& McCann. M (1975). Personal effectiveness: Guiding people to assert themselves and improve their social skills. Champaiọn, Illinois: Research Press.

Lindsay, W. R. \& Stoffelmayr, B. E. (1982). The concept of generalisation in behaviour therapy. Behavioural Psychotherapy, 10, 346-355.

Lindsay, W. R., Symons, R. S. \& Sweet, T (1979). A programme for teaching social skills to socially inept adolescents: A description and evaluation. Journal of Adolescence, 2, 215-218.

Linehan, M. M., Golfried, M. R., \& Goldfried, A. P. (1979). Assertive therapy: Skill trainina or cognitive restructuring? Behaviour Therapy, $10,372-388$. 
Luborsky, L.. Sinqer, B. \& Luborsky, L. 11975). Comparative studies of the psychotherapies: Is it true that everyone has won and all must have prizes? Archives of General Psychiatry, 32, 995-1008.

MacMillan, A. S., Kolvin, I., Garside, R. F., Nicol, A. R. and Leitch, I. M. (1980). Psychological Medicine, 10, 265-276.

Marzillier, J. S. \& winter, K. (1978). Success and failure in social skills training: Individual differences. Behaviour Research and Therapy, 16 , $67-84$

Matson; J.L, Rotatori, A.F. \& Helsel, W.J. (1983). Development of a rating scale to measure social skills in children; the Matson Evaluation of Social skills with Younasters. Behaviour, Research and Therapy, 21, 4, $335-340$.

McFall, R.M. (1982). A review and reformulation of the concept of social skills. Behavioural Assessment, $4,1-33$.

McGurk, B. J. and Newell. T. C. (1981). Social skills training with a sex offender. The Psychological Record, 31, 277-283.

Mersch, P.P.A., Emmelkamp., P. M. G., Bogels, S. M. \& van der Sleen, J. (1989). Social phobia: Individual response patterns and the effect of behavioural and cognitive interventions. Behaviour Research and Therapy, 27, 421-434.

Michelson, L., Mannarino, A. P., Marchione, K. E., Stern, M., Figueroa, J. \& Beck. S. (1983). A comparative outcome study of behavioural socialskills training, interpersonal problem-solving, and non-directive control treatments with child psychiatric outpatients. Behaviour Research and Therapy, 21, 545-556.

Mischel, W. (1968) Personality and assessment. New York: Wiley.

Mize, J. M. \& Ladd, G. W. (1990). A coqnitive-social learning approach to social skill trainina with low-status preschool children. Developmental Psychology, 26, 388-397. 
Montemayor, R., Adams, G. R. \& Gulotta, T. B (Eds.) (1990). From childhood to adolescence - a transitional period? London: Sage.

Newton, A., Kindness K., \& McFadeyn. M. (1983). Patients and social skills qroups: Do they lack social skills? Behavioural Psychotherapy, 11, 116126.

O'Connor, R. D. (1969). Modification of social withdrawal through symbolic modellinq. Journal of Applied Behaviour Analysis, 2, 15-22.

O'Connor, R. D. (1972). Relative efficacy of modelling, shapina, and the combined procedure for modification of social withdrawal. Journal of Abnormal Psycholocy, 79, 327-334.

Oden, S. \& Asher, S. (1977). Coaching children in social skills for friendship makina. Child Development, 48, 495-506.

Parloff, M. B., Waskow, I. E. \& Wolfe, B. E. (1978). Research on therapist varlables in relation to process and outcome. In S. L. Garfield \& A. E. Bergin (Eds.) Handbook of psychotherapy \& behaviour change: An empirical analysis. (Sec. ed.) New York: Hiley.

Patterson, G. R. \& Reid. J. B. (1970) Reciprocity and coercion: Two facets of social systems. In C. Neurinaer \& J. D. Michael, (Eds.) Behaviour modification in clinical osychology. New York: Appleton-Century-Crofts.

Payne, P., and Halford, W.K., (1990) Social Skills Training with Chronic Schizophrenic Patients living in Community Settings. Behavioural Psychotherapy, 18, $49-64$.

Pelleqrini, D. S. \& Urbain, E. S. (1985). An evaluation of interpersonal coonitive problem-solving with children. Journal of Child Psychology and Psychiatry, 26, 17-41.

Pendleton, L. R., Shelton, J. L., \& Hilson, S. E. (1976). Social interaction training using systematic homework. The Personnel and Guidance Journal, 54, 484-7.

Pentz, M. A. (1981). The contribution of individual differences to assertion training outcome in adolescents. Journal of Counselling Psychology, 28, 529-532. 
Phillips, L., \& Zialer, E., (1964). Role orientation, the action-thought dimension and outcome in psychiatric disorder. Journal of Abnormal and Social Psycholoqy, 68, 381-389.

Putallaz, M. (1983). Predictina children's sociometric status from their behaviour. Child Development, 54, 1417-1426.

Rachman, S. J. \& Wilson G. T. (1980). The effects of psychological therapy (Sec. Ed.). Oxford: Perqamon.

Rinn, R.C. \& Markle, A. (1979). Modification of social skills deficits in children. In A. S. Bellak \& M. Hersen (Eds.) Research and practice in social skills training. New York: Plenum.

Robson, C. (1983). Experiment, design, and statistics in psychology (Sec. Ed.). Harmondsworth: Penquin Books.

Roff, M., Sells, B., \& Golden, M. M. (1972). Social adjustment and personality development in children. Minneapolis: University of Minneapolis Press.

Renwick, S. \& Emler, N. (1991). The relationship between social skills deficits and juvenile delinquency. British Journal of Clinical Psychology, $30,61-71$.

Rogers, C. R. (1957). The necessary and sufficient conditions of therapeutic personality chanqe. Journal of Consulting Psychology, 21, $95-103$.

Ross, D., Ross, S. \& Evans, T. A. (1971). The modification of extreme social withdrawal by modification with guided practice. Journal of Behaviour Therapy and Experimental Psychiatry, 2, 273-279.

Rutter, M. (1967). A children's behaviour questionnaire for completion by teachers: Preliminary findings. Journal of Child Psychology and Psychiatry, 8, 1-11.

Rutter, M. (1985). Family and school influences on behavioural development. Journal of Child Psychology and Psychiatry, 26, 349-368. 
Sacks S. \& Gaylord-Ross. R, (1389). Peer mediated and teacher directed social skills training for visually impaired students. Behaviour Therapy, 20, 619-638.

Santer, H. (1983). Social skills training. Newsletter of the British Association for Behavioural Psychotherapy, 9, 12-14.

Sarason, I. G. (1978). A coanitive social learning approach to juvenile delinquency. In R. Hare and D. Schalling (Eds.) Psychopathic Behaviour: Approaches to research. London: Wiley.

Sarason, I. G. \& Sarason, B. R. (1981). Teachina coqnitive \& social skills to high school students. Journal of Consulting and Clinical Psychology, $49,908-918$.

Searle, J. R. (1975). Indirect speech acts. In P. Cole and J. L. Morgan (Eds.) Syntax and semantics (Vol. 3) Speech acts. New York: Seminar Press.

Seligman, M. E. P. (1975). Helplessness. San Francisco: Freeman.

Shepherd, G. (1983). Introduction. In S. Spence \& G. Shepherd (EdS.) Developments in Social Skills Training. London: Academic Press.

Shure, M. B. \& Spivack, G. (1980). Interpersonal problem-solving as a mediator of behavioural adjustment in preschool and kindergarten children. Journal of Applied Developmental Psychology, 1, 29-44.

Siege1, S. (1956). Non-parametric statistics for the behavioural sciences. London: MCGraw-Hill.

Spence, S. (1980). Social skills training with children and adolescents: A cousellor's manual. Windsor: NFER.

Spence, S. (1981). Differences in social skills performances between institutionalized iuvenile male offenders and a comparable group of boys without offence records. British Journal of Social Psychology, 20, 163171. 
Spence, S. H. (1983). Teaching social skills to children. Journal of Child Psychology and Psychiatry, 24, 621-627.

Spence. S.H. \& Marzillier. J.S. (1981). Social skills training with adolescent male offenders II. Short-term. long-term and aeneralised effects. Behaviour Research and Therapy, 19, $349-368$.

Steiner, I.D., 1955. Interpersonal behaviour as influenced by accuracy of social perception. Psychological Review, 62, 268-274.

Strain, P. S., Shores R. E. \& Kerr M. M .(1976). An experimental analysis of spillover effects on the social interaction of behaviourally handicapped preschool children. Journal of Applied Behaviour Analysis, 9, $31-40$.

Stravynski, A., Marks, I. \& Yule, W. (1982). Social skills problems in neurotic outpatients: Social skills training with and without cognitive modification. Archives of General Psychiatry, 39, 1378-1385.

Tiffen, K. \& Spence, S. H. (1986). Responsiveness of isolated versus rejected children to social skills training. Journal of Child Psychology and Psychiatry, 27, 343-355.

Trower, P.(1979). Fundamentals of interpersonal behaviour: A socialpsychological perspective. In A. S. Bellack \& M. Hersen (Eds.) Research and practice in social skills training. New York: Plenum Press.

Trower, P. (1982). Towards a qenerative model of social skills: A critique and synthesis. In J. P. Curran \& P. M. Monti (Eds.) Social Skills Trainina - A practical handbook for assessment and treatment. NeW York: Guildford Press.

Trower, P. (Ed.) (1984). Radical approaches to social skills training. London : Croom Helm.

Trower, P., Bryant, B. \& Argyle, M. (1978). Social skills and mental health. London: Methuen. 
Truax, C. B. \& Mitchell. K. M. (1971). Research on certain therapist interpersonal skills in relation to process and outcome. In A. E. Bergin \& S. L. Garfield (Eds.), Handbook of psychotherapy and behaviour change: An empirical analysis. New York: Wiley.

Truax, C. B. \& Carkhuff, R. R. (1971) Towards effective counselling and psychotherapy: Training and practice. Chicaqo: Aldine.

Twentyman , C., Boland, T. \& MCFall, R.M. (1981). Heterosocial avoidance in colleqe males. Behaviour Modification, 5, 523-552.

Tyler, P. \& Tapsfield, P. (1984). Review of self ratina devices in social skills assessment, and the preliminary investigation of a new scale (S.O.C.S.I.T). Behavioural Psychotherapy, 12, 223-236.

Ullman, C. A. (1957). Teachers, peers, and tests as predictors of adjustment. Journal of Educational Psychology, 48, 257-267.

Van Hasselt, V. B., Hersen, M. \& Bellack, A. S. (1981). The validity of role play tests for assessing social skills in children. Behaviour Therapy, 12, 202-216.

Van Hasselt, V. B., Hersen, M., Whitehill, M. B. \& Bellack, A. S (1979). Social skill assessment and training for chidren: An evaluative review. Behaviour Research and Therapy, 17, 4113-4439.

Verduyn, C. M., Lord., W. \& Forrest, G. C. (1990). Social skills training in schools: An evaluation study. Journal of Adolescence, 13, 3-16.

Walker, H. M., Greenwood, C. R., Hops, H. \& Todd, N. M. (1979). Differential effects of reinforcing topoqraphic components of social interaction: Analysis and direct replication. Behaviour Modification, $3,291-321$.

Watzlawick, P., Weakland, J., \& Fisch, M. D. (1974). Change: Principles of problem formation and problem resolution. New York: Norton. 
Wheeler, Y. A. \& Ladd, G. W. (1982). Assessment of childeren's selfefficacy for social interaction with peers. Developmental Psychology, $18,795-805$.

White, R. W. (1959). Motivation reconsidered - The concept of competence. Psychological Review, 66, 297-333.

Wilkins, W, (1986). Therapy-therapist confounds in psychotherapy research. Cognitive Therapy and Research, 10, 3-11.

Willems, E. P. (1973). Go ye into all the world and modify behaviour: An ecologist's view. Representative Research in Social Psychology, 4, 93105.

Williamson, D. A., Moody, S. C., Granberry, S. W., Lethermon, V. R. \& Blouin, D. C. (1983). Criterion related validity of a roleplay social skills test for children. Behaviour Therapy, 14, 466-481.

Winter, K. \& Marzillier, J. S. (1983). Psychiatric outpatients. In S. Spence \& G. Shepherd (Eds.) Developments in social skills training. London: Academic Press.

Wolpe, J. (1969). The practice of behaviour therapy. New York: Pergamum Press.

Yardley, K. M. (1979). Social skills training - A critique. British Journal of Medical Psychology, 52, 55-62.

Youniss, J. (1980). Parents and peers in social development. Illinois, University of Chicaqo Press.

Zigler, E. \& Philips, L. (1961). Social competence and outcome in psychiatric disorder. Journal of Abnormal and Social Psychology, 63, $264-271$ 


\section{ACKNOWLEDGEMENTS}

I am grateful to Joan Tyler. Jim Barnes, Julie Hudson, Tony Beall and Paul Drake for their assistance with the Social skills Trainina; and to Ray Stirling for his encouragement and sunport of the research project. 